Contact and Friction in Systems with Fibre Reinforced Elastomers

N. V. Rodríguez Pareja 
De promotiecommissie is als volgt samengesteld:

Voorzitter en secretaris:

prof.dr. F. Eising

Promotor:

prof.dr.ir. D.J. Schipper

Assistent Promotor:

dr.ir. M.A. Masen

Leden

prof.dr.ir. P. de Baets

prof.dr.ir. S. Franklin

prof.dr.ir. R. Akkerman

prof.dr.ir. J.W.M. Noordermeer

prof.dr.ir. G.J. Vancso
Universiteit Twente

Universiteit Twente

Universiteit Twente

Universiteit Gent, België

University of Sheffield, United Kingdom

Universiteit Twente

Universiteit Twente

Universiteit Twente

The studies described in this thesis are part of the Research Programme of the Dutch Polymer Institute, P.O. Box 902, 5600 AX Eindhoven, The Netherlands, project nr. \#664.

Rodríguez Pareja, Natalia Valentina

Contact and Friction in Systems with Fibre Reinforced Elastomers

Ph.D. Thesis, University of Twente, Enschede, The Netherlands.

October 2012

ISBN 978-90-365-3454-3

DOI 10.3990./1.9789036534543

Printed by Ipskamp Drukkers B.V., Enschede, The Netherlands.

Keywords: Reinforced elastomer, contact, friction, surface layer, anisotropy, viscoelasticity.

Cover designed by Higher for Hire

Copyright (c) 2012 by N.V. Rodríguez Pareja, Enschede, The Netherlands.

All rights reserved 


\title{
Contact and Friction in Systems with Fibre Reinforced Elastomers
}

\author{
PROEFSCHRIFT \\ ter verkrijging van \\ de graad van doctor aan de Universiteit Twente, \\ op gezag van de rector magnificus, \\ prof. dr. H. Brinksma, \\ volgens besluit van het College voor Promoties \\ in het openbaar te verdedigen \\ op vrijdag 26 oktober 2012 om 14.45 uur
}

\author{
door \\ Natalia Valentina Rodríguez Pareja \\ geboren op 27 december 1983 \\ te Valparaiso, Chili.
}


Dit proefschrift is goedgekeurd door: de promotor: prof.dr.ir. D.J. Schipper de assistent promotor: dr.ir. M.A. Masen 


\section{Summary}

The tribological behaviour (contact and friction) of systems that include fibre reinforced elastomers is studied in this thesis. The elastomer composite is considered to behave as a viscoelastic anisotropic continuum material.

In the defined tribo-system, the most influential friction mechanism is adhesion. Therefore the size of the contact area during sliding and the shear stresses in the contact area are studied.

A contact model that considered the viscoelasticity of the elastomer and the anisotropy caused by the directionality of the fibres has been developed to describe the size of the contact area in the static case. To obtain the size of the contact area during sliding, sliding experiments were performed and from these results a function that relates the static and dynamic contact area was proposed.

Due to sliding interaction the surface of the elastomer is modified, this modification of the surface is called a tribo-generated surface layer. This layer has poor mechanichal properties when compared to the bulk of the elastomer and influences the tribological behaviour of tribo-systems with elastomers. The friction is considered to be caused by shearing this layer.

The shear stresses occurring in the tribo-generated surface layer during sliding contact are modelled using a shear stress model which considers viscoelastic behaviour of the interfacial layer. The viscoelastic behaviour was modelled by a Maxwell model of two elements. Properties of the surface layer are obtained from indentation and friction measurements.

The friction model corresponds closely with friction experiments for the unreinforced and reinforced EPDM at different sliding velocities. In the case of the reinforced EPDM a lower coefficient of friction was found, this is the result of a decrease on the contact area due to the reinforcement. 



\section{Samenvatting}

Dit proefschrift behandelt het tribologisch gedrag (contact en wrijving) van systemen waarin één van de materialen een vezelversterkt elastomeer is. Deze elastomeer composiet gedraagt zich als een visco-elastisch, anisotroop continuüm.

In het gedefinieerde tribo-systeem is adhesie het meest belangrijke wrijvingsmechanisme, daarom worden in dit werk de afmetingen van het contactvlak in glijdend contact en de schuifspanningen in het contact bestudeerd.

Voor het beschrijven van de afmeting van het contactvlak in statische contactsituaties is een contactmodel ontwikkeld, waarin zowel de viscoelasticiteit van het elastomeer als de anisotropie, die het gevolg is van de oriëntatie van de vezels, worden meegenomen. Met behulp van experimenten zijn vervolgens de contactafmetingen tijdens glijdend contact bepaald en uit de resultaten is een vergelijking afgeleid die het verband tussen de afmetingen van het statische en het dynamische contactvlak beschrijft.

Als gevolg van het glijdende contact treden veranderingen op aan het oppervlak van het elastomeer. Deze tribologisch gegenereerde oppervlaktelaag heeft verminderde mechanische eigenschappen in vergelijking met het bulkmateriaal van het elastomeer en heeft een invloed op het tribologisch gedrag ervan. De wrijving in het contact wordt veroorzaakt door het afschuiven van deze laag.

De schuifspanningen in de tribologisch gegenereerde oppervlaktelaag tijdens glijdend contact worden beschreven met een model dat het viscoelastisch gedrag van de oppervlaktelaag beschrijft. Het visco-elastisch gedrag is gemodelleerd met een Maxwell model met twee elementen. De mechanische eigenschappen van de oppervlaktelaag zijn bepaald met behulp van indentatie- en wrijvingsmetingen.

De resultaten van het wrijvingsmodel bij verschillende glijsnelheden komen overeen met wrijvingsexperimenten voor zowel EPDM zonder vezels als voor vezelversterkt EPDM. Voor versterkt EPDM wordt een lagere wrijving gevonden, die veroorzaakt wordt door de kleinere contactafmetingen als gevolg van de versteviging van het materiaal. 



\section{Table of contents}

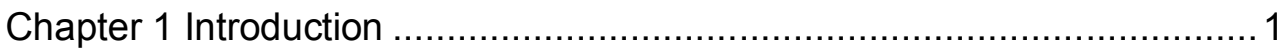

1.1 Fibre reinforced elastomers....................................................... 1

1.2 Composite materials ............................................................... 2

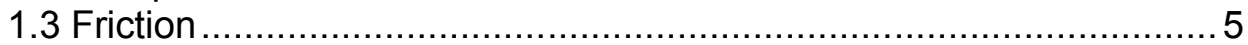

1.4 Objectives of this research ..................................................... 7

1.5 Outline of this thesis.................................................................. 7

Chapter 2 Sliding friction and fibre reinforced elastomers ...................... 9

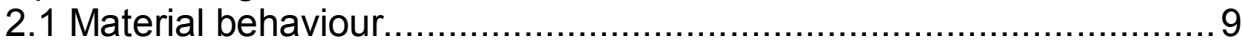

2.2 Contact modelling ................................................................... 14

2.3 Mechanisms involved in sliding friction ...................................... 21

2.4 Summary ........................................................................... 30

Chapter 3 The tribological system in this thesis ..................................... 31

3.1 Material behaviour of the contacting bodies .............................. 32

3.2 Interaction between the contacting surfaces.................................. 38

3.3 Mechanisms of sliding friction .............................................. 44

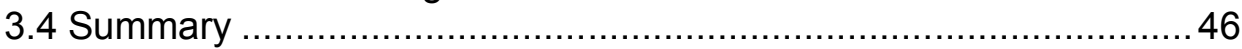

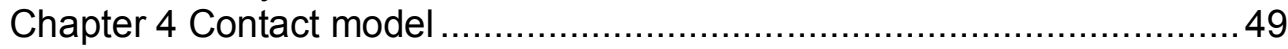

4.1 The static contact area .......................................................... 51

4.2 The dynamic contact area ................................................... 77

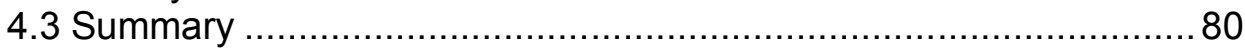

Chapter 5 Shear stresses in the contact ........................................... 83

5.1 Development of surface layer due to friction................................. 84

5.2 Modelling the behaviour of the interfacial layer............................ 95

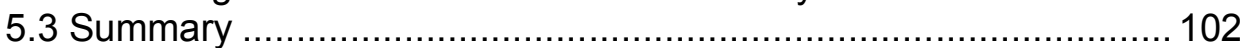

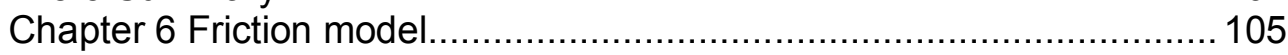

6.1 Friction model and parameter study ...................................... 105

6.2 Friction behaviour of fibre reinforced EPDM .............................. 111

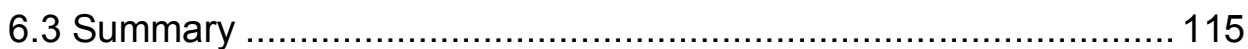

Chapter 7 Conclusions and recommendations.................................. 117

7.1 Overview of this thesis .......................................................... 117

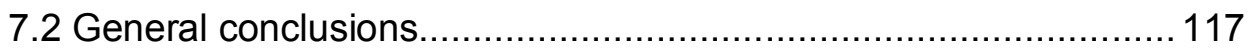

7.3 Discussion and recommendations....................................... 120

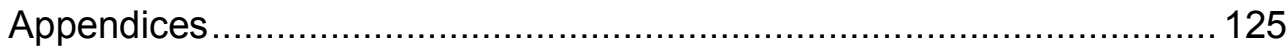

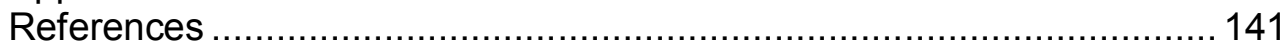




\section{Nomenclature}

\begin{tabular}{|c|c|c|}
\hline \multicolumn{3}{|c|}{ Roman Symbols } \\
\hline$\overline{A_{\text {static }}}$ & Contact area in the static case & {$\left[\mathrm{m}^{2}\right]$} \\
\hline$A_{\text {sliding }}$ & Contact area during sliding & {$\left[\mathrm{m}^{2}\right]$} \\
\hline$A_{\text {unidirectional }}$ & $\begin{array}{l}\text { Contact area calculated with the unidirectional } \\
\text { contact model }\end{array}$ & {$\left[\mathrm{m}^{2}\right]$} \\
\hline$A_{\text {anisotropic }}$ & $\begin{array}{l}\text { Contact area calculated with the fully - anisotropic } \\
\text { contact model }\end{array}$ & {$\left[\mathrm{m}^{2}\right]$} \\
\hline$A_{v>>1 \mathrm{~m} / \mathrm{s}}$ & Contact area at very high velocities & {$\left[\mathrm{m}^{2}\right]$} \\
\hline$A_{f}$ & Frontal projection of the contact area & {$\left[\mathrm{m}^{2}\right]$} \\
\hline$a_{x}, a_{y}$ & Half widths of the elliptical contact area & {$[\mathrm{m}]$} \\
\hline$a_{x}(t), a_{y}(t)$ & Time dependent half widths of the contact area & {$[\mathrm{m}]$} \\
\hline $\bar{a}(t)$ & Dimensionless time dependent contact area & {$[-]$} \\
\hline$a(t)$ & Time dependent radius of the contact area & {$[\mathrm{m}]$} \\
\hline [C] & Stiffness matrix & [Pa] \\
\hline$C_{i j k l}$ & Stiffness tensor & [Pa] \\
\hline$C$ & Constant & {$[-]$} \\
\hline$D$ & Deborah number & {$[-]$} \\
\hline$E$ & Elastic modulus & [Pa] \\
\hline$E_{*}$ & Equivalent elastic modulus & {$[\mathrm{Pa}]$} \\
\hline$E_{*_{Z}}$ & $\begin{array}{l}\text { Equivalent elastic modulus in the indentation } \\
\text { direction }\end{array}$ & {$[\mathrm{Pa}]$} \\
\hline$E_{x}, E_{y}, E_{z}$ & Elastic modulus in $x, y$ and $z$ direction respectively & {$[\mathrm{Pa}]$} \\
\hline$F$ & Total friction force & {$[\mathrm{N}]$} \\
\hline$F_{p}$ & Friction force due to deformation (ploughing) & {$[\mathrm{N}]$} \\
\hline$F_{a}$ & Friction force due to adhesion & {$[\mathrm{N}]$} \\
\hline$F_{N}$ & Applied normal force & {$[\mathrm{N}]$} \\
\hline$F_{N}(t)$ & Time dependent applied normal force & {$[\mathrm{N}]$} \\
\hline $\bar{F}(t)$ & Dimensionless time dependent applied normal force & {$[-]$} \\
\hline$f(t)$ & Auxiliary function $\mathrm{f}(t)=\bar{p}(t) / \bar{a}(t)$ & {$[-]$} \\
\hline$f^{-1}(t)$ & Inverse of the auxiliary function $f(t)$ & {$[-]$} \\
\hline G & Shear modulus & {$[\mathrm{Pa}]$} \\
\hline $\begin{array}{l}G_{y z,} G_{z x} \\
G_{x y}\end{array}$ & $\begin{array}{l}\text { Shear modulus in the planes } y z, z x \text { and } x y \text {, } \\
\text { respectively }\end{array}$ & {$[\mathrm{Pa}]$} \\
\hline$G_{L}$ & Shear modulus of the surface layer & {$[\mathrm{Pa}]$} \\
\hline$G^{\prime}$ & Storage modulus in shear & {$[\mathrm{Pa}]$} \\
\hline G” & Loss modulus in shear & {$[\mathrm{Pa}]$} \\
\hline$G_{e}$ & Equilibrium modulus & {$[\mathrm{Pa}]$} \\
\hline$G_{i}$ & Relaxation strength coefficient i & [Pa] \\
\hline
\end{tabular}




\begin{tabular}{|c|c|c|}
\hline$H(t)$ & Heaviside function & {$[-]$} \\
\hline$h$ & Thickness of the surface layer & [m] \\
\hline$I_{1}, I_{2}$ & $\begin{array}{l}\text { Contour integrals that encapsulate the material } \\
\text { properties in an anisotropic material, defining the } \\
\text { contact behaviour }\end{array}$ & {$\left[\mathrm{Pa}^{-1}\right]$} \\
\hline$L$ & Length of the contact patch & [m] \\
\hline$n$ & $\begin{array}{l}\text { n parameter of Elsharkawy - Hamrock Shear model, } \\
\text { in the present thesis } n=1.05\end{array}$ & {$[-]$} \\
\hline $\bar{P}$ & Load parameter (adhesion map) & {$[-]$} \\
\hline $\bar{p}(t)$ & Dimensionless time dependent pressure distribution & {$[-]$} \\
\hline$p_{0}$ & Maximum contact pressure & {$[\mathrm{Pa}]$} \\
\hline$p_{m}$ & Mean contact pressure & {$[\mathrm{Pa}]$} \\
\hline$R$ & Radius of the indenter & [m] \\
\hline$R_{a}$ & Arithmetic mean value of the surface roughness & [m] \\
\hline [S] & Compliance matrix & {$\left[\mathrm{Pa}^{-1}\right]$} \\
\hline$S^{*}$ & Time in which $a(t)=a$ & {$[\mathrm{~s}]$} \\
\hline$s$ & Variable in the Laplace domain & \\
\hline$T_{g}$ & Glass transition temperature & {$\left[{ }^{\circ} \mathrm{C}\right]$} \\
\hline$T^{9}$ & Temperature & {$\left[{ }^{\circ} \mathrm{C}\right]$} \\
\hline$t$ & Time & [s] \\
\hline$t_{*}$ & Dummy variable of the convolution integral & [s] \\
\hline$U$ & Mean velocity, during sliding is $v / 2$ & {$\left[\mathrm{~m} \mathrm{~s}^{-1}\right]$} \\
\hline $\boldsymbol{u}$ & Displacement field vector & [m] \\
\hline$u_{i}\left(x_{i}\right)$ & Displacement field depending on spatial variables & {$[\mathrm{m}]$} \\
\hline $\bar{u}(t)$ & Normalized time function of the displacement field & {$[-]$} \\
\hline$\tilde{u}_{z}$ & $\begin{array}{l}\text { Fourier transform of the normal component of the } \\
\text { displacement field }\end{array}$ & \\
\hline$u_{z}$ & Normal component of the displacement field & {$[\mathrm{m}]$} \\
\hline$v$ & Sliding velocity & {$\left[\mathrm{m} \mathrm{s}^{-1}\right]$} \\
\hline$v_{c a r}$ & Velocity of the car & {$\left[\mathrm{m} \mathrm{s}^{-1}\right]$} \\
\hline$\Delta \mathrm{v}$ & Difference of velocity between the car and the road & {$\left[\mathrm{m} \mathrm{s}^{-1}\right]$} \\
\hline$W$ & $\begin{array}{l}\text { Work of adhesion between the surfaces (adhesion } \\
\text { map) }\end{array}$ & {$\left[\mathrm{J} \mathrm{m}^{-2}\right]$} \\
\hline$X_{\text {boundary }}$ & $\begin{array}{l}\text { Dimensionless maximum } x \text { coordinate for a given } y \text {, } \\
X_{\text {boundary }}=x_{\text {boundary }} / a_{x}\end{array}$ & {$[-]$} \\
\hline$x_{\text {boundary }}$ & $\begin{array}{l}\text { Maximum } x \text { coordinate which a point in the contact } \\
\text { area can have for a given } y\end{array}$ & [m] \\
\hline$x, y, z$ & $\begin{array}{l}\text { Spatial variables, } x \text { and } y \text { are in the indentation } \\
\text { plane and } z \text { is along the direction of indentation }\end{array}$ & \\
\hline$z_{0}$ & Equilibrium separation between atomic planes & [m] \\
\hline
\end{tabular}




\begin{tabular}{|c|c|c|}
\hline \multicolumn{3}{|c|}{ Greek Symbols } \\
\hline$\alpha$ & Roughness parameter, $\alpha=R_{a} R / a^{2}$ & {$[-]$} \\
\hline$\dot{\gamma}$ & Strain rate in shear, also called shear rate & {$\left[\mathrm{s}^{-1}\right]$} \\
\hline$\delta(t)$ & Time dependent indentation depth & [m] \\
\hline$\delta$ & Indentation depth & {$[\mathrm{m}]$} \\
\hline$\delta(x)$ & Dirac delta function & \\
\hline$\varepsilon(t)$ & Time dependent strain & {$[-]$} \\
\hline$\varepsilon_{i j}, \boldsymbol{\varepsilon}$ & Strain tensor & {$[-]$} \\
\hline$\eta_{i}$ & Viscosity i, $\tau_{i}=\eta_{i} / G_{i}$ & {$[\mathrm{~Pa} \mathrm{~s}]$} \\
\hline$\eta_{L}$ & Viscosity of the surface layer & {$[\mathrm{Pa} \mathrm{s}]$} \\
\hline$\lambda$ & Elasticity parameter (adhesion map) & {$[-]$} \\
\hline$\lambda_{i}$ & Retardation time of the creep compliance coefficient $i$ & [s] \\
\hline$\mu$ & Coefficient of friction & {$[-]$} \\
\hline$\mu_{a}$ & Coefficient of friction due to the adhesion mechanism & {$[-]$} \\
\hline$\mu_{\text {exp }}$ & Coefficient of friction obtained from experiments & {$[-]$} \\
\hline$\mu$ & Elastic Parameter $\lambda=1.16 \mu$ (adhesion map) & {$[-]$} \\
\hline$v$ & Poisson's ratio & {$[-]$} \\
\hline$\xi$ & Dimensionless viscosity parameter $\xi=\eta_{L} \dot{\gamma} / \tau_{L}$ & {$[-]$} \\
\hline$\sigma(t)$ & Time dependent stress & {$[\mathrm{Pa}]$} \\
\hline$\sigma_{i j}, \boldsymbol{\sigma}$ & Stress tensor & {$[\mathrm{Pa}]$} \\
\hline$\sigma_{t}$ & $\begin{array}{l}\text { Average stress in the tangential direction (along } \\
\text { sliding) }\end{array}$ & {$[\mathrm{Pa}]$} \\
\hline$\sigma_{n}$ & $\begin{array}{l}\text { Average stress in the normal direction (along the } \\
\text { indentation) }\end{array}$ & {$[\mathrm{Pa}]$} \\
\hline$\tau_{i}$ & Relaxation time of the stress relaxation coefficient & [s] \\
\hline$\tau$ & Shear stress between the contacting surfaces & {$[\mathrm{Pa}]$} \\
\hline$\dot{\tau}$ & Variation of shear stress with time & {$[\mathrm{Pa}]$} \\
\hline $\bar{\tau}$ & Dimensionless shear stress $\bar{\tau}=\tau / \tau_{L}$ & {$[-]$} \\
\hline$\tau_{L}$ & Limiting shear stress & {$[\mathrm{Pa}]$} \\
\hline$\phi(t)$ & Creep compliance function & {$\left[\mathrm{Pa}^{-1}\right]$} \\
\hline$\phi_{z}(t)$ & $\begin{array}{l}\text { Creep compliance function in the direction of } \\
\text { indentation }\end{array}$ & {$\left[\mathrm{Pa}^{-1}\right]$} \\
\hline
\end{tabular}




\begin{tabular}{clc}
\hline $\bar{\phi}(t)$ & Normalized creep compliance & {$[-]$} \\
$\phi_{r}$ & Creep compliance at fully relaxed state & {$\left[\mathrm{Pa}^{-1}\right]$} \\
$\phi_{i}$ & Creep compliance coefficient i & {$\left[\mathrm{Pa}^{-1}\right]$} \\
$\psi(t)$ & Stress relaxation function & {$[\mathrm{Pa}]$} \\
$\psi_{z}(t)$ & Stress relaxation function in the direction of & {$[\mathrm{Pa}]$} \\
$\bar{\psi}(t)$ & indentation & {$[-]$} \\
$\psi_{i j k l}(t)$ & Normalized stress relaxation & Stress relaxation of the material in 3-D \\
$\omega$ & Frequency & {$[\mathrm{Pa}]$} \\
$\omega$ & Rotational velocity & {$[\mathrm{Hz}]$} \\
\hline & & {$\left[\mathrm{rad} \mathrm{s}{ }^{-1}\right]$} \\
\hline & & \\
\hline EPDM & Ethylene propylene diene rubber \\
DMA & Dynamic mechanical analysis & \\
SEM & Scanning electron microscope \\
PU & Polyurethane & \\
PMMA & Poly(methyl methacrylate) & \\
PC & Polycarbonate & \\
L & Longitudinally oriented fibres \\
R & Randomly oriented fibres \\
OVI & Orthotropic Viscoelastic Isochronous contact model & \\
\hline & &
\end{tabular}





\section{Chapter 1 Introduction}

Elastomeric materials are used in daily life in a variety of applications, such as tyres, v-belts and hoses. An important reason for their use is their capability to withstand large deformations without permanent loss of shape, structure and mechanical properties. In contrast to this flexibility, their tribological behaviour is often poor, characterised by high friction in dynamic contact situations and a rather low abrasion wear resistance. One way to solve this is to add fibres to the elastomeric material: the fibres improve the stiffness and strength of the elastomer while maintaining a level of flexibility. A typical example of this is a transmission belt, but possible applications also include seals, engine mounts and energyefficient tyres with reduced rolling resistance.

In this work, the contact and friction behaviour of such fibre reinforced elastomers is discussed; a contact model that incorporates both viscoelastic and anisotropic behaviour is developed. With this, the friction is modelled by describing the shear behaviour of the sliding contact.

In the present chapter, fibre reinforced elastomers and composite materials are introduced, followed by a definition of the tribological system and the type of friction in study and the objectives of this research.

\subsection{Fibre reinforced elastomers}

The advantage of fibre reinforced elastomers composite is its ability to tailor physical characteristics, e.g. stiffness and deformation, obtaining a more flexible material when compared with other types of polymeric matrices.

As fibre reinforced elastomers present flexibility and strength they are suitable to use in flexible and compliant structures. The applications include adaptive and inflatable structures, biomechanical devices and rubber muscle actuators.

The specific characteristics of a fibre reinforced elastomer composite will depend on the elastomer and fibre selection as well as on the length of the fibres, the concentration of the fibres and their orientation.

As the material properties of the composite are modified, the tribological behaviour of a tribo-system with this kind of composite will also be affected by the concentration and direction of the fibres. 
In this thesis, a short-cut fibre reinforced elastomer is studied. The reinforcing fibres are high-performance fibres such as aramids, they are short fibres and they will be oriented unidirectionally and randomly in the elastomer matrix. To study the frictional behaviour of this composite material, a tribological system must be defined. But first an introduction on composite materials and their classifications will be given.

\subsection{Composite materials}

A composite is a structural material that combines, at macroscopic level, two or more materials not soluble in each other. One of the constituents is called matrix. The mechanical properties of the matrix will improve by embedding the other constituent, called the reinforcing phase [1].

The use of composite materials started because of the lack of a single material that has all the properties required for a particular task. A historical example is the adobe brick used in constructions. These bricks were made of clay, water and an organic material such as straw or bamboo shoots. The latter allowed the clay to bind and dry evenly. Many constructions were made with this reinforced material (for example Egyptian constructions from 1500 B.C.). Examples of modern composites are glass fibre reinforced resins used in aeronautical constructions.

Nowadays composites are chosen for their beneficial costs, weight and properties (thermal, electrical, surface topography, strength, stiffness).

Composite materials can be classified by the geometry of their fillers (flakes, fibres and particles) and by their matrix (metals, ceramics and polymers).

\subsubsection{Composites classified by filler}

Fillers can have three different types of geometry. Fig. 1.1 shows, from top to bottom, flakes, fibres and particles. A detailed explanation of each type of filler will also be presented. 
Introduction

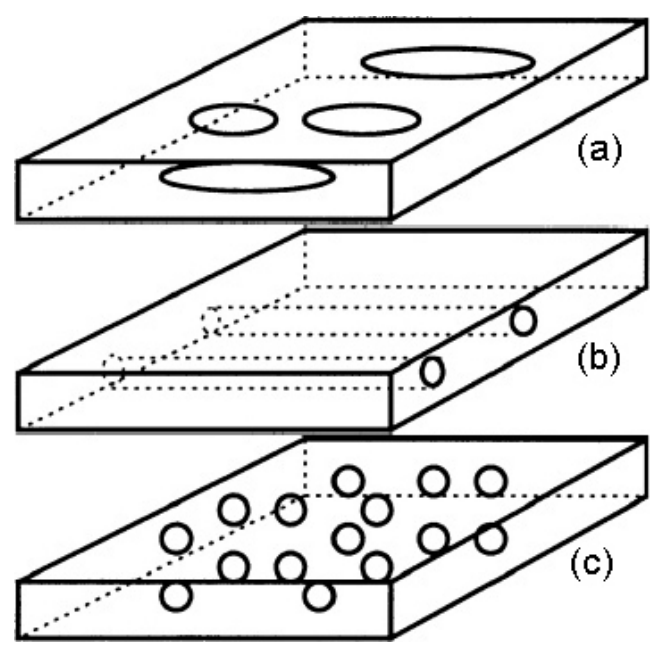

Fig. 1.1: Composites with different shapes of filler: (a) flakes, (b) long fibres and (c) particles, see [1].

\section{Flake composites}

Flake composites, Fig. 1.1 (a), consist of matrices reinforced by flat fillers such as glass, mica, aluminium and silver. The advantages of flake fillers are that they provide higher resistance to deflect under out-of-plane bending and have greater strength while still being cost-effective. The disadvantage is the difficulty of orienting the flakes, which is why composite materials with this kind of filler are not very common.

\section{Fibre composites}

These composites are formed by short or long fibres embedded in a matrix, Fig. 1.1 (b). Long and continuous fibres are easy to orient and process and offer high impact resistance, low shrinkage, improved surface finish, etc. Short fibres are more difficult to orient, but they offer a cost effective solution and are easy to produce and to work with. Fibres are made with different cross-sectional shapes. Circular fibres are the most common due to easy handling and manufacturing. The material of the fibres is very relevant for the final properties of the composite. To improve the mechanical properties of the composite, fibres are expected to have high strength and elastic properties. Another factor that influences the composite performance is the orientation of the fibres. If the fibres have one preferred orientation in the composite, the strength and stiffness of the composite will be very high in that direction. Orientation configurations of fibre composites range from randomly oriented and unidirectionally oriented to more 
complex configuration such as stacked woven fibre laminas. Fibres used in composite materials include polyester fibres, glass, aramid and carbon. The last three fibres are "high performance" fibres, which have been especially engineered for uses that require very high strength, and high resistance to extreme temperature and/or chemical resistance.

\section{Particulate composites}

Particulate composites, Fig. 1.1 (c), are composed of small particles embedded in a matrix. Because the particles are added randomly, the final composite behaves isotropically. The advantages of this kind of filler are typically improved strength, increased operating temperature, oxidation resistance etc. Disc brake pads are one excellent example of this kind of composite. They consist of ceramic particles embedded in a soft metallic matrix.

\subsubsection{Composites classified by matrix}

\section{Metallic matrix}

These are composites in which the matrix is made of a metal such as aluminium, magnesium or titanium. The main advantage of metal composites is that they increase the specific strength and modulus of low density metals like aluminium and titanium (the matrix). Also by adding fibres with a low coefficient of thermal expansion it is possible to reduce the thermal expansion of the matrix itself. Metal composites are commonly used when high elastic properties, service temperature and conductivity are needed.

\section{Ceramic matrix}

The advantages of using a ceramic matrix are its high strength, very high service temperature, low density and chemical inertness. By reinforcing a ceramic matrix it is possible to obtain a material with the properties mentioned before but with higher fracture toughness. The typical applications of these composites are at high-temperature working conditions and where high elastic properties are needed, e.g. cutting tool inserts for high-temperature environments.

\section{Polymer matrix}

Polymer matrices are the most common matrices used in composite material due to their high strength and simple manufacturability at low costs. Their common disadvantages are low operating temperature and low 
Introduction

elastic properties, which can be improved by adding a second phase. Examples of polymer matrices are, epoxy, polyester and urethane.

\subsection{Friction}

A tribological system includes two surfaces in contact, moving relative to each other. The environment can influence the moving contact with its pressure, temperature and humidity and by the presence of an intermediate material between the surfaces in contact, such as oil, water, air and particles, resulting in changes to the tribological behaviour of the moving contacting surfaces. Tribological behaviour refers to the behaviour of the surfaces in contact and relative motion regarding friction, wear and lubrication. In this thesis, friction of fibre reinforced elastomers is studied.

Friction is the resistance to motion during sliding and/or rolling, experienced by one solid moving tangentially over another solid with which it is in contact. The tangential load that resists the movement, and thus works in the opposite direction of the motion, is referred to as the friction force. The friction is a system response and cannot be considered a material property, so it will always depend on both contacting surfaces and their environment. There are two types of friction:

\section{Static friction}

Static friction refers to the friction produced when the bodies or surfaces in contact are not in relative motion. The static friction force is equal to the force applied to produce motion. Its maximum value is reached just before the start of movement.

\section{Dynamic friction}

Dynamic friction applies to the contacting bodies or surfaces that are already in relative motion. The tangential force required to maintain the contacting surfaces in motion is called dynamic or kinetic friction force. Depending on the contact situation, i.e. rolling and/or sliding, the friction between the contacting surfaces is called rolling, sliding or rolling to sliding friction. In this thesis, friction refers to sliding friction unless stated otherwise.

\subsubsection{Sliding friction of tribo-systems including fibre reinforced elastomers}

Sliding friction in tribological systems involving an elastomer is a complex phenomenon influenced by sliding velocity, applied load and the ambient temperature of the tribological system. Furthermore, the addition of fibres to 
the elastomers adds anisotropy to the influencing factors of the tribological behaviour of the system. Studies considering the influence of the fibre orientation on the tribo-systems including polymer composites are Mens [2], Blanchet [3], Shim [4], Lancaster [5] and Sung [6]. Studies regarding the influence of fibre orientation on the behaviour of a tribo-system that includes elastomer composites are more limited, for example the work of Wada and Uchiyama [7] that studies the wear and the work of Uchiyama et al. [8] that studies friction. These two studies, concerning polymers and elastomers, agree on the large influence of the fibre orientation in relation to the sliding direction on the friction and/or the wear of the tribo-system.

Shim [4] found that a lower friction coefficient is observed when the fibres are aligned along the sliding direction. To explain the low friction, it was shown that the shear resistance along the fibres is lower than the shear resistance in the cross section of the fibres, and therefore the tangential force needed to maintain the motion is less.

Mens [2] and Lancaster [5] studied the modification of the tribological behaviour for different polymers reinforced with different fibres. In all cases the addition of the fibres changed the tribological properties of the system, but in different ways for each composite.

Sung [6] studied three different fibre reinforced polymer composites. With a certain combination of fibre-matrix he found that the fibres oriented normal to the sliding direction offered the lowest friction and wear. For the other fibre-matrix combination, the longitudinal direction offered the lowest friction and wear behaviour. It was considered that in composites containing a ductile matrix incorporating short fibres, oriented normal to the sliding direction, the sliding process will deform the matrix causing an alignment of the fibres near the surface. As the alignment increases, the shear resistance decreases and the reduction of friction and wear is explained. The experimental observations of Wada and Uchiyama [7] agree with the previous results. They showed that for a short fibre reinforced elastomer the lowest wear occurs when the fibres are aligned perpendicular to the sliding direction, and in the friction study of Uchiyama et al. [8] they show that friction coefficient decreases in the case of reinforced rubber in which the orientation of the fibres is normal to the contact. This corresponds to a reduced contact area (in relation to other orientations) and - due to alignment of the fibres in the surface - lower shear resistance. In this thesis, the influence of certain factors on the tribological behaviour of fibre reinforced elastomers will be studied. However, it is necessary first to define a tribological system. 
Introduction

\subsubsection{Definition of the tribo-system in this work}

In this thesis, the tribological behaviour of a system in which one of the materials is a fibre reinforced elastomer will be studied. The elastomer is modelled as a homogeneous material, meaning that the fibres are homogeneously distributed.

An example of such tribo-system is tyre-road contact. This type of contact is characterized by a relatively large contact area. This allows the effects of the fibre size in comparison with the contact area to be ignored. Therefore the material response of the composite can be considered as the mechanical response of a continuum.

\subsection{Objectives of this research}

In this thesis, the tribological behaviour, i.e. contact and friction of short fibre reinforced elastomers in contact with a rigid surface, is studied. The short fibres are assumed to be unidirectionally oriented or randomly oriented, to be homogeneously distributed and to have negligible dimensions when compared to the size of the contact area. Therefore the composite is considered to behave as a continuous viscoelastic anisotropic material. The objectives of this research are twofold:

- Development of an experimentally validated physical model by which the single asperity contact between the studied elastomeric composite against a rigid indenter is described;

- Development of an experimentally validated physical model by which the friction between the elastomeric composite sliding against a rigid counter surface is described.

With these models one is able to optimize the tribological behaviour of the system in which the elastomeric composite operates.

\subsection{Outline of this thesis}

This thesis is composed of seven chapters. Chapter 2 presents a literature review on the parameters involved in sliding friction of fibre reinforced elastomers in contact with a smooth surface. The tribological system studied in this thesis is discussed in Chapter 3. Chapter 4 is devoted to the development of a new contact model that considers viscoelastic and anisotropic behaviour of fibre reinforced elastomers as well as the effect of the sliding on the size of the contact area. Chapter 5 presents an analysis of the modification of the surface properties due to sliding friction. A shear 
Chapter 1

stress model for the layer is proposed. In Chapter 6, the models developed in Chapter 4 and 5 are coupled in order to describe the frictional behaviour of tribo-systems containing fibre reinforced elastomers. Guidelines are given regarding the optimal orientation of the fibres. Finally, in Chapter 7, the conclusions of this thesis are presented, and recommendations for future research are proposed. 


\section{Chapter 2 Sliding friction and fibre reinforced elastomers}

Sliding friction is related to the tangential forces that oppose the sliding motion of the moving surfaces in contact. An important aspect of friction is the contact between the two surfaces, i.e. the contact area and pressure as a function of external load, material properties, macro/micro geometry and surface energy. This chapter will present an overview of the factors influencing the contact area between two surfaces, the mechanisms involved in sliding friction and the factors influencing the sliding friction in systems in which (fibre reinforced) elastomers are involved.

\subsection{Material behaviour}

\subsubsection{Viscoelastic material behaviour}

Viscoelastic materials have intermediate behaviour between the response of an elastic solid and a viscous liquid. An elastic material will strain instantly when a stress is applied. The resistance to strain of the viscous material will follow a certain time function while being stressed. Many viscous materials strain linearly with time when a stress is applied, so a linear time function is common in modelling the viscosity. Therefore viscoelastic materials are characterized by having an instantaneous elastic response under an applied stress combined with a linearly time-dependent viscous response. This response depends on the temperature of the viscoelastic material. The material properties of a viscoelastic material can vary as a function of temperature. At low temperatures they approach elastic behaviour and at these temperatures the viscoelastic material is in the "glassy" state. At high temperatures the viscoelastic material becomes softer and its properties decrease rapidly with increasing temperature; this state is called the flow region. At temperatures between the high and low extremes, corresponding to the above-mentioned regions, the material behaves as a mixture. In this state the elastomer is commonly in use; this is called the elastomeric region [9], see Fig. 2.1. 


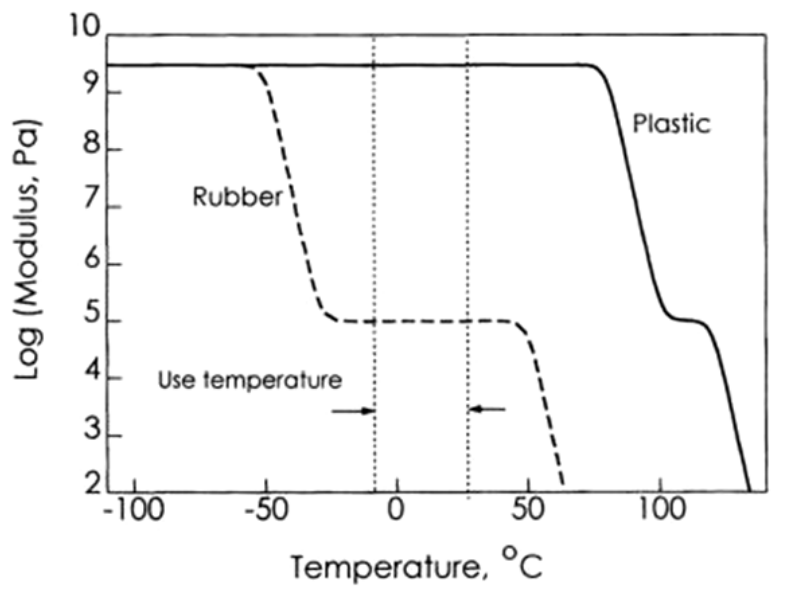

Fig. 2.1: Comparison between rubbers and plastics and the dependence of their modulus on temperature [9].

Due to the viscous character of this material, there are time-dependent phenomena that characterize this material: hysteresis, stress relaxation and creep compliance.

\section{Hysteresis}

Due to the viscous characteristic of these materials, the energy used to deform the body is not fully recuperated as is the case for ideally elastic materials. This phenomenon is called hysteresis. The amount of energy stored in the deformation (during loading) is not completely used to restore the original shape (unloading). This dissipated energy is called hysteresis loss and corresponds to the area between the loading and unloading curve in a stress-strain curve (see Fig. 2.2). 


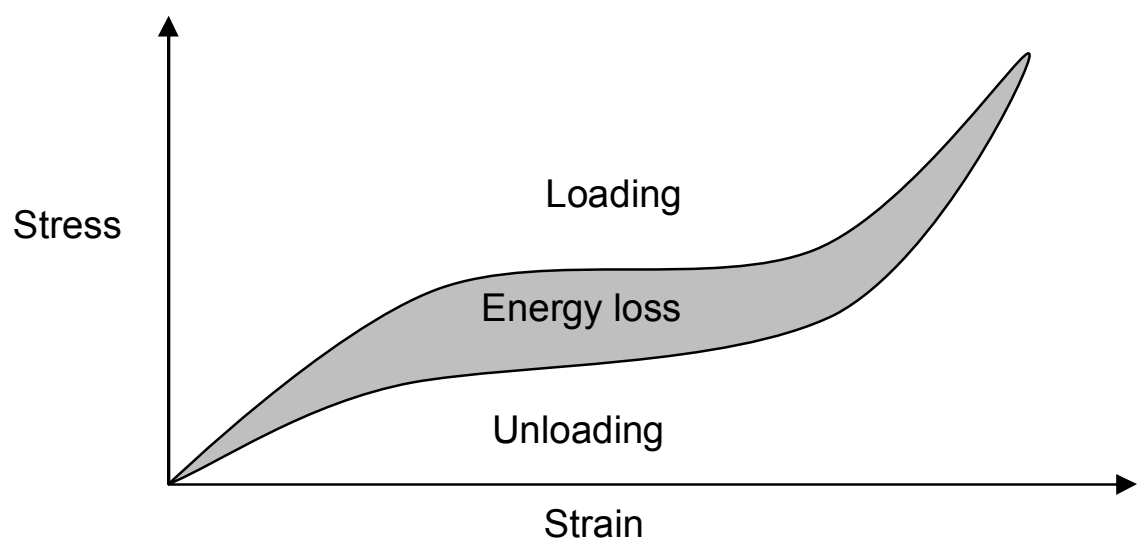

Fig. 2.2: Stress-strain curve $(\sigma-\varepsilon)$ schematic for elastomers, the grey area between the lines of loading and unloading is a measure of the energy loss of a loading cycle.

\section{Stress relaxation}

An elastic body under a constant strain, $\varepsilon_{0}$, shows a constant stress, $\sigma_{0}$. This is not the case for viscoelastic bodies. Under a constant instantaneous applied strain $\varepsilon_{0}$, the stress reaches a maximum, $\sigma_{0}$, and then diminishes with time, $\sigma(t)$. This phenomenon is called stress relaxation. Considering linear viscoelasticity the relaxation modulus $\psi(t)$ is defined as:

$$
\psi(t)=\frac{\sigma(t)}{\varepsilon_{0}}
$$

\section{Creep}

If a stress, $\sigma_{0}$, is applied instantaneously to a viscoelastic body and is held constant, the strain will increase with time, $\varepsilon(t)$. This phenomenon is called creep. The ratio between strain and stress is called compliance. When the strain varies with time and the stress is held constant the ratio is referred to as the creep compliance $\phi(t)$, and is defined as:

$$
\phi(t)=\frac{\varepsilon(t)}{\sigma_{0}}
$$

An example of creep compliance is given in Fig. 4.7. 


\subsubsection{Anisotropic material behaviour}

As mentioned before, adding fibres in the material influences the mechanical properties of the composite. Within the different fibre-related factors (length, orientation, shape and material) which contribute to the mechanical properties of the composite, the orientation factor is also very relevant, not only because it changes the material properties but also because it affects the type of medium. Due to their amorphous configuration, elastomeric materials can be modelled as an isotropic medium while the addition of fibres with a preferred orientation changes the material response in different orientations. Therefore the composite material must be considered as an anisotropic media.

Anisotropic materials are defined by 21 independent elastic constants. Knowing the 21 independent constants will allow the relation between stress and strain to be determined. Full anisotropy is rare because many natural and synthetic materials have material symmetry, i.e. elastic properties are identical in the directions of symmetry. This symmetry reduces the number of independent elastic constants by establishing relations within the stiffness $(C)$ and the compliance $(S)$ matrices [11]. Stiffness and compliance matrices are defined by Hooke's law in three dimensions in Eq. 2.3 and Eq. 2.4 respectevely. In one dimension, the stiffness matrix $\mathrm{C}$ becomes the elastic modulus.

$$
\begin{gathered}
\sigma=[C] \varepsilon \\
\varepsilon=[S] \sigma
\end{gathered}
$$

When a material contains three mutually perpendicular planes of symmetry the material is called an orthotropic material and can be defined by only nine independent elastic constants. This kind of anisotropic material is one of the more common anisotropic materials and examples of it are a wooden bar and rolled steel. The stiffness matrix of this kind of material is shown in Eq. 2.5, where $E$ indicates the elastic modulus, $G$ indicates the shear modulus and $v$ indicates the Poisson's ratio. The stiffness matrices are symmetrical $v_{x y} / E_{x}=v_{y x} / E_{y}$ and so on. The nine elastic constants are $\mathrm{E}_{\mathrm{x}}$, $E_{y}, E_{z}, G_{y z}, G_{z x}, G_{x y}, v_{y z}, v_{z x}, v_{x y}$. 


$$
[S]=\left[\begin{array}{cccccc}
\frac{1}{E_{x}} & -\frac{v_{x y}}{E_{x}} & -\frac{v_{x z}}{E_{x}} & 0 & 0 & 0 \\
-\frac{v_{y x}}{E_{y}} & \frac{1}{E_{y}} & -\frac{v_{y z}}{E_{y}} & 0 & 0 & 0 \\
-\frac{v_{z x}}{E_{z}} & -\frac{v_{z y}}{E_{z}} & \frac{1}{E_{z}} & 0 & 0 & 0 \\
0 & 0 & 0 & \frac{1}{G_{y z}} & 0 & 0 \\
0 & 0 & 0 & 0 & \frac{1}{G_{z x}} & 0 \\
0 & 0 & 0 & 0 & 0 & \frac{1}{G_{x y}}
\end{array}\right]
$$

If one plane of an orthotropic material behaves isotropically, the material is called transversally isotropic and is defined by only five independent elastic constants.

If all the planes of the orthotropic material are isotropic, the material is called isotropic; it is then defined by only two independent elastic constants and has an infinite number of principal directions. This is the most common symmetry (steel, iron and rubber). The stiffness matrix in the isotropic case has the following form:

$$
[S]=\left[\begin{array}{cccccc}
\frac{1}{E} & -\frac{v}{E} & -\frac{v}{E} & 0 & 0 & 0 \\
-\frac{v}{E} & \frac{1}{E} & -\frac{v}{E} & 0 & 0 & 0 \\
-\frac{v}{E} & -\frac{v}{E} & \frac{1}{E} & 0 & 0 & 0 \\
0 & 0 & 0 & \frac{2(1+v)}{E} & 0 & 0 \\
0 & 0 & 0 & 0 & \frac{2(1+v)}{E} & 0 \\
0 & 0 & 0 & 0 & 0 & \frac{2(1+v)}{E}
\end{array}\right]
$$




\subsection{Contact modelling}

The contact between the surfaces is important in determining the friction between the surfaces when they are in relative motion. To model the contact between the surfaces there are various models available in literature that include part of the material behaviour of a fibre reinforced elastomer. These models are explained in terms of the material behaviour that they consider.

\subsubsection{Isotropic elastic material behaviour}

The contact area between two elastic ellipsoids in contact (Hertzian contact) is dependent on the elastic modulus and the Poisson's ratio of both materials in contact, the radius of curvature of the ellipsoids at the point in contact and the applied load [10]. The Hertz model for two ellipsoids in contact is summarized in Appendix B.

The Hertz contact model describes the contact behaviour considering:

- the surfaces in contact are smooth, continuous and non-conforming, and therefore the stresses are finite in the whole space;

- the surfaces are isotropic and therefore the deformations are the same in all directions;

- the contact between the surfaces is frictionless;

- the surfaces are represented as elastic half spaces. Hence it is possible to calculate local deformations. This consideration means that the size of contact area is much smaller than the radii of curvature of the bodies. This ensures that outside the contact area the surface behaves like a half space and that the size of the contact area is smaller than the dimensions of the bodies in contact which avoids influence of boundaries of the bodies in the stress field.

Where these assumptions do not apply, for instance for material couples that have high adhesion, the Hertz model does not give an accurate description of the contact behaviour and an alternative model has to be considered.

\subsubsection{Isotropic viscoelastic material behaviour}

Because in a viscoelastic material the material properties are no longer constant, as assumed in the Hertz model, it is necessary to consider 
alternative contact models which consider the viscoelastic behaviour. Lee and Radok [11] derived a viscoelastic contact model based on the model of Hertz. They calculated the pressure distribution inside the contact area for the case of a rigid sphere pressed against a viscoelastic half space with a monotonic increasing contact area. While giving a first indication about the contact parameters, a more general solution was needed since the contact area may change differently with time. The contact between a viscoelastic asperity and a rigid one was modelled by Johnson [10]. The solution considering viscoelasticity was presented in the cases of controlled load and controlled indentation. If the deformation history is known, the viscoelastic solution is found by replacing the elastic parameters with the viscoelastic parameters.

Graham [12] formulated the mixed boundary problem in the quasi-static linear theory of viscoelasticity. He determined the expressions for the displacement and stress field produced in an isotropic half space by the action of an arbitrarily and time-dependent applied pressure. Later [13] Graham considers that instead of having an arbitrary time-dependent distribution of pressure, the known parameter is the contact area, also following an arbitrarily chosen function. A solution can be found as long as the contact area corresponds to a function with any number of maxima and minima, where the minima occurs in zero and the maxima follows an monotonic increasing function. Using these contact models it is possible to describe not only the contact area and depth of one indentation, but also the time-dependent contact area after repeated indentations.

A contact model that includes all previous solutions was developed by Ting [14], by subsequently solving the contact area and pressure field and combining these to a general solution. In case of loading a viscoelastic isotropic material with an spherical rigid indenter, the pressure distribution and the contact area are defined by Eq. 2.7 and Eq. 2.8 respectively, where $\psi(t)$ denotes the stress relaxation function, $\phi(t)$ the creep compliance function, $\mathrm{R}$ corresponds to the radius of the spherical indenter, a corresponds to the radius of the contact area and $\mathrm{F}$ corresponds to the total applied force.

$$
\begin{gathered}
p(r, t)=\frac{4}{\pi R} \int_{0}^{t} \psi\left(t-t^{\prime}\right) \frac{d}{d t^{\prime}}\left\{a^{2}\left(t^{\prime}\right)-r^{2}\right\}^{1 / 2} d t^{\prime} \\
a^{3}(t)=\frac{3}{8} R \int_{0}^{t} \phi\left(t-t^{\prime}\right) \frac{d}{d t^{\prime}} F\left(t^{\prime}\right) d t^{\prime}
\end{gathered}
$$




\subsubsection{Anisotropic elastic material behaviour}

As an anisotropic material has different material properties in different directions, the contact area between a hard indenter and an anisotropic material will be different from the contact area with an isotropic material.

The contact model developed by Hertz assumes isotropic materials. This means that the strain-stress relations of the materials in contact were defined by only two elastic constants. When the materials are not isotropic, properties in more than one direction need to be known. For orthotropic materials, the strain-stress relations can be determined by knowing nine elastic constants. For transversally isotropic bodies, the strain-stress relation is determined by five elastic constants. Studies considering the contact behaviour of transversally isotropic bodies were done for the two dimensional case by Green and Zerna [15] where the indenter is a rigid punch. Dahan and Zarka [16] studied the elastic contact of a sphere with a transversely isotropic half space. They obtained an analytical expression for the stress along the axis of the indenter and on the surface. Yang and Sun [17] and Tan and Sun [18] assumed that the contact area and pressure in the case of an orthotropic material could be derived from the isotropic case, replacing the isotropic modulus by the orthotropic one in the loading direction. Willis [19] developed a method to calculate the contact area of a completely anisotropic material by numerically solving a contour integral. Swanson [20] used the method of Willis to solve the Hertzian contact for orthotropic bodies.

Using these contact models, significant differences between the contact areas in the isotropic and anisotropic cases are found. The contact model of Willis [19] for fully anisotropic materials and the model of Swanson [20] for orthotropic materials show that in cases where the reinforcement occurs in one direction of the plane of indentation, the contact area has an elliptical shape. Let's say that the indentation occurs in the direction $z$. If the reinforcement occurs in direction $x$ or $y$, the contact area is no longer a circle but an ellipse with its principal axes along the directions $x$ and $y$. The model of Sun et al. [17,18], Willis [19] and Swanson [20] agree in the case of an increase of reinforcement in the direction $z$; they find that the contact area decreases with an increase of the reinforcement but remains circular.

\subsubsection{Other factors influencing the contact area}

Besides the material behaviour of the contacting surfaces as discussed before, there are other factors that influence the contact area. They can be related to the material behaviour of one of the contacting bodies or to the tribological system and are therefore related to both materials and the 
contact conditions between them. These factors will be explained in more detail later.

\subsubsection{Sliding velocity}

For elastic materials, the contact area and shape during sliding remain constant and are independent of the sliding speed. This is not the case for materials that do not behave elastically. For example, the contact area between a rigid sphere and a plastic deforming material is circular in the static case, while it is semi-circular when sliding occurs. The actual contact area of the contact remains the same in both situations, therefore it is possible to calculate the contact area during sliding derived from the static case.

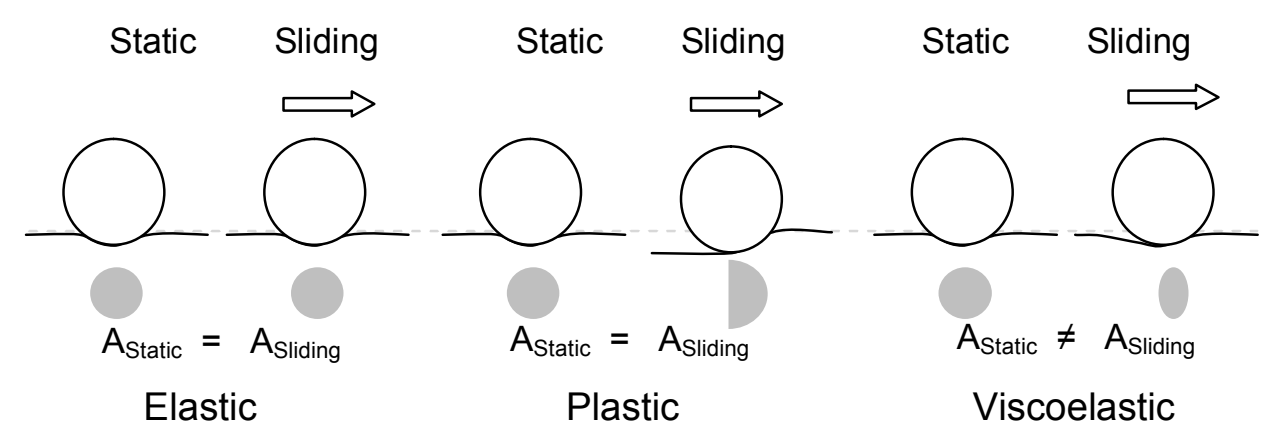

Fig. 2.3 Variation of the shape and contact area in static and sliding contact for materials behaving (from left to right) elastically, plastically and viscoelastically.

In the case of viscoelastic materials, both the shape and the contact area during sliding differ from the static case. Studies [21, 22-25] have shown a variation in the shape of the contact areas during different sliding conditions. Fig. 2.4 shows, for example, the contact area between a smooth elastomer and a microscope glass sliding at increasing speed. A change in the shape and the size can be seen. The value of S.F. under the pictures refers to the ratio of the axes of the ellipse. 


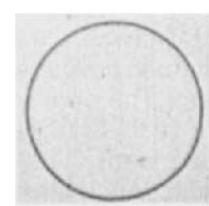

S.F. $=1$

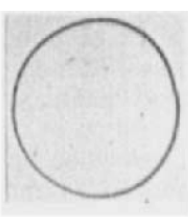

S.F. $=1.07$

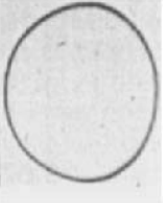

S.F. $=1.15$

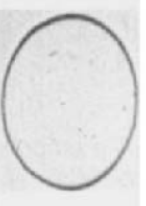

S.F. $=1.29$

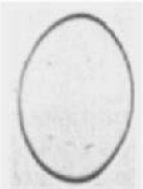

S.F. $=1.45$

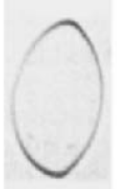

S.F. $=1.7$

Fig. 2.4: Contact area from static to sliding. It can be seen that the shape of the contact area changes from circular contact in the static case to elliptical contact in the sliding case. S.F. $=1$ to 1.7 [25].

At low sliding velocities the contact is semi-static, meaning that the contact area is equal to the static contact area and remains constant until a certain velocity is reached. At a certain velocity the contact area starts to decrease as shown in Ludema and Tabor [21], Fig. 2.5, and Vorvolakos and Chaudhury [26], Fig. 2.7. According to Ludema and Tabor [21], as the sliding speed increases, the deformation rate increases resulting in a decrease of the contact area and an increase of the stiffness of the rubber.

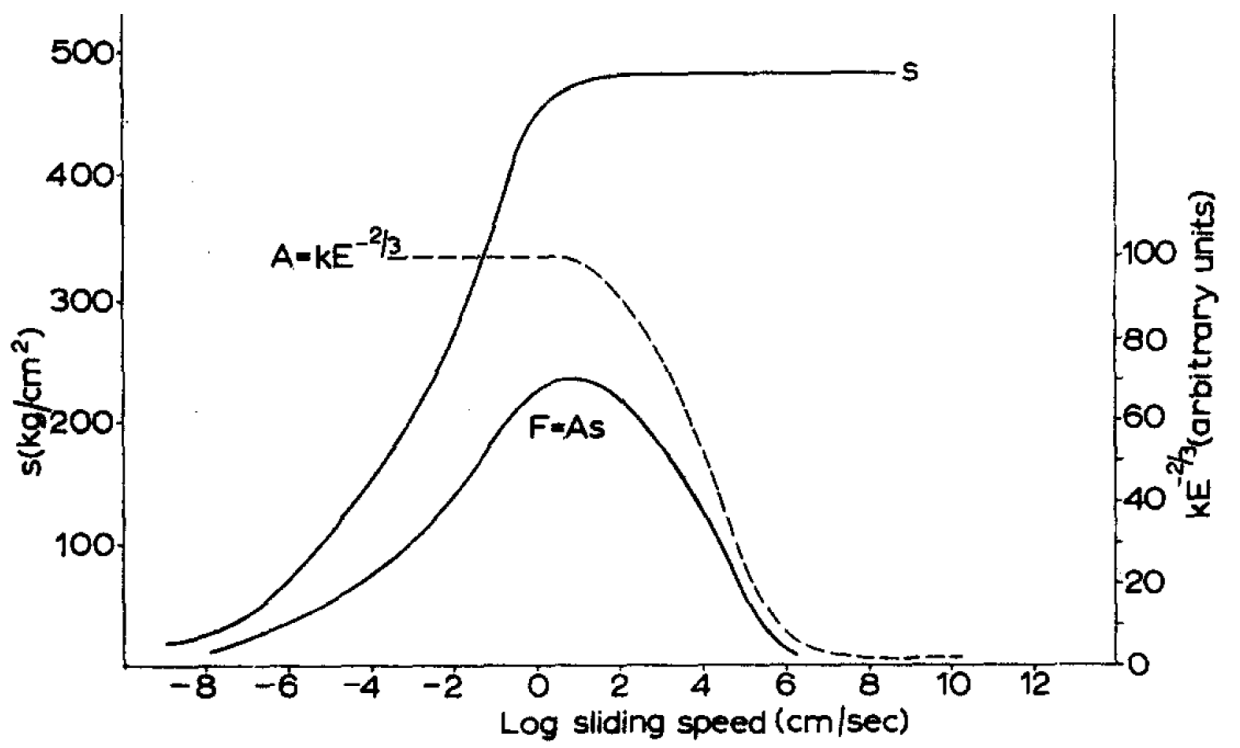

Fig. 2.5: Variation of shear stresses $(\mathrm{s})$ and contact area $(\mathrm{A})$ as a function of sliding speed [21]. The product of both $s$ and $A$ is a measure of the friction force. 
Sliding friction and fibre reinforced elastomers

\subsubsection{Adhesion}

In some cases, the contact area between elastic surfaces is larger than the one predicted by Hertz model. The mechanism contributing to this effect is the adhesion between the contacting surfaces: the greater the adhesion, the greater the contact area. This increase in the contact area, will also affect the frictional behaviour.

There are several models available in literarture that can be used to describe the contact behaviour including the effect of adhesion. The model of Johnson, Kendall and Roberts [27] considers that attractive forces act in the contact area, increasing its size. In contrast, Derjaguin, Muller and Toporov [28] model the adhesive contact by taking the Hertzian contact area, with the attractive forces acting in the vicinity of the contact area, changing the pressure distribution. The model developed by Maugis [29], based on Dugdale's approximation of adhesive stresses, is an intermediate adhesion model in which the JKR and DMT models are considered as extreme cases.

Johnson and Greenwood [30] made a comparison between these models, summarized in the adhesion map as shown in Fig. 2.6 . This map provides a quick method to select the applicable model for certain contact conditions by calculating an elasticity parameter, $\lambda$ (Eq. 2.9) and a normal load parameter, $\bar{P}$ (Eq. 2.10).

$$
\begin{gathered}
\lambda=1.16 \mu \\
\mu=\left(\frac{R W^{2}}{E_{*}^{2} z_{0}^{3}}\right)^{1 / 3} \\
\bar{P}=\frac{F_{N}}{\pi W R}
\end{gathered}
$$

Where $F_{N}$ denotes the applied normal load, $W$ is the work of adhesion between the surfaces, $R$ is the radius of the indenter, $z_{0}$ is the equilibrium separation between atomic planes and $E *$ denotes the equivalent elastic modulus. The elastic parameter, $\mu$, denotes the ratio of the elastic displacement of the surfaces at the point of separation (pull off) to the effective range of surface forces characterized by $z_{0}$, and the load parameter is a measure of the ratio of the load to the adhesive pull-off force. 


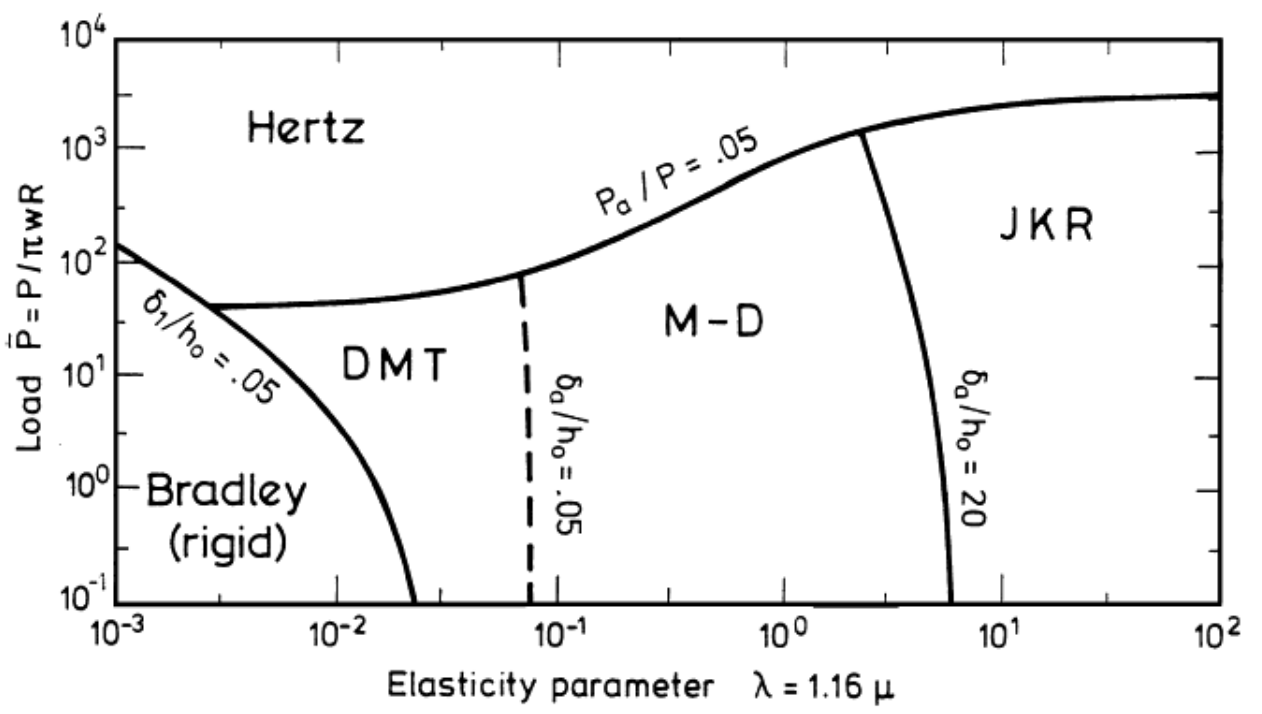

Fig. 2.6: Adhesion map, made by Greenwood and Johnson [30]. The $x$ axis shows the elasticity parameter and the $y$ axis shows the load parameter.

In the case of soft materials such as elastomers, according to Greenwood and Johnson [30] the elasticity parameter exceeds $10^{2}$. Therefore the contact model often used for elastomers is the JKR contact model. Vorvolakos and Chaudhury [26] measured the contact area in sliding between a silicone rubber and a hard lens, and compared it with the contact area calculated using the JKR contact model, see Fig. 2.7. The elasticity parameter they calculated for their contact was $\lambda \approx 1.9 \cdot 10^{3}$ and the load parameter $\bar{P} \approx 130$, indicating that the suitable contact model, according to the adhesion map (Fig. 2.6), is JKR. The results from the adhesion map, in the study of Vorvolakos and Chaudhury [26], agree with their experimental observation in the static case. During sliding, no changes were observed at very low velocities but after increasing the velocity above a certain value, the measured contact area decreased to values closer to the Hertz model, see Fig. 2.7. This indicates that at higher velocities, and depending on the position of the load parameter in the adhesion map, the Hertzian contact model approximates the size of the contact area between the surfaces better. 
Sliding friction and fibre reinforced elastomers

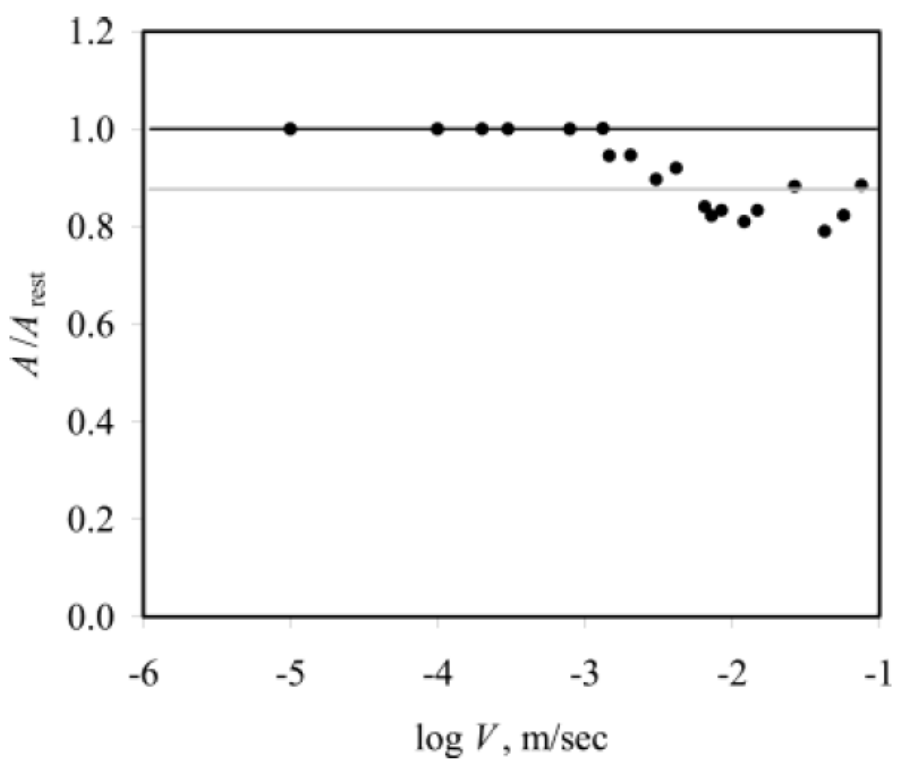

Fig. 2.7: Ratio between the sliding and the static contact areas, as a function of the sliding velocity. The black line indicates the ratio of contact area in static and dynamic case, calculated using the JKR contact model $\left(A_{\text {measured }} / A_{J K R}\right)$. The grey line indicates the ratio of contact area calculated by Hertz and JKR $\left(A_{\text {Hertz }} / A_{J K R}\right)$,

[26].

\subsection{Mechanisms involved in sliding friction}

In early investigations of friction, it was believed that the dynamic friction has its source in the mechanical interaction between the asperities of the materials in contact. Later, it was found that as friction is a dissipative force, some other mechanisms ensure energy loss. These mechanisms can be divided in two main categories: adhesion and deformation.

\subsubsection{Adhesion}

When two rough surfaces are in contact under a certain load, the real contact takes place at the summits of asperities. The contacting asperities might interact, creating bonds between each other. As a result, the tangential forces generated during sliding must be high enough to be able to shear these bonds and to maintain the relative motion between the surfaces. After the bonds of two contacting asperities are broken, new bonds are created in new contacting asperities. The attractive forces generated by the formed bonds have their origin in chemical and physical 
interaction. Chemical interactions generally refer to covalent, ionic or metallic bonds as well as hydrogen bonds. Physical interaction involves van der Waals bonds. The last ones are generally weaker than the chemical ones but they are always present between two asperities that are in close proximity to each other. Van der Waals bonds and hydrogen bonds are found to be the most important causes of adhesion in polymers [31].

As the created bonds between surfaces are the effect of molecular forces, they can be of the same character as the bonds in the material itself. Therefore the strength of the adhesion in the interface of the two bodies in contact may be stronger than the bonds in the bulk of the materials. Shear will cause the weakest bonds to break. If the weakest bonds are those in the interface, the adhesive friction will be determined by the shear strength of the interface and when the weakest bonds are those in the bulk of the material, the adhesive friction is determined by the shear strength of the bulk, meaning that the surface will wear [31].

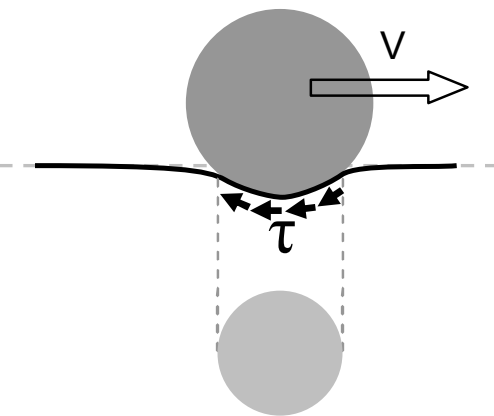

A

Fig. 2.8 Adhesion originates by attractive forces between the surfaces in contact, and is defined by the shear stresses within the contact area.

The friction force produced by adhesion, for dry contact, is described by Bowden and Tabor, 1950 [31]:

$$
F_{a}=\tau A
$$

where $\tau$ is the shear stress and $A$ is the real contact area between the contacting surfaces, as seen in Fig. 2.8. The adhesive strength can be decreased by reducing the surface interaction in the interface, by applying contaminants or a very thin lubricant layer.

In the case of materials with a high Young's Modulus, such as metals and ceramics, the real contact area is only a small fraction of the apparent 
nominal contact area. Under certain conditions, these materials can have low adhesion strength. Polymers and especially elastomers, on the other hand, have higher ratios of real/apparent contact area, which generally results in stronger adhesion.

\subsubsection{Deformation}

The friction present during sliding of two bodies in contact is caused by adhesion and also by deformation. The deformation can occur at different scales, due to microscopic and macroscopic interactions. The first type of interaction refers mainly to plastic deformation of the interlocking surface asperities. The second type of interaction refers to deformation of the softer material as a result of harder asperities ploughing through it [31]. The ploughing asperities can belong to the surface of the contacting body, or can be wear particles formed during the sliding process.

In the case of ideally elastic deformation, meaning when the hard asperities only cause a small indentation, the ploughing is not permanent and the surface goes back to its original shape when the pressure is released. Therefore there is no energy loss. In the case of plastic deformation, the ploughing is permanent and a groove is created, generating energy losses. The friction force caused by deformation or ploughing, for plastic contact, is given by:

$$
F_{p}=\sigma_{t} A_{f}
$$

where $\sigma_{t}$ refers to the average stress in the tangential direction and $A_{f}$ refers to the frontal projection of the contact area.

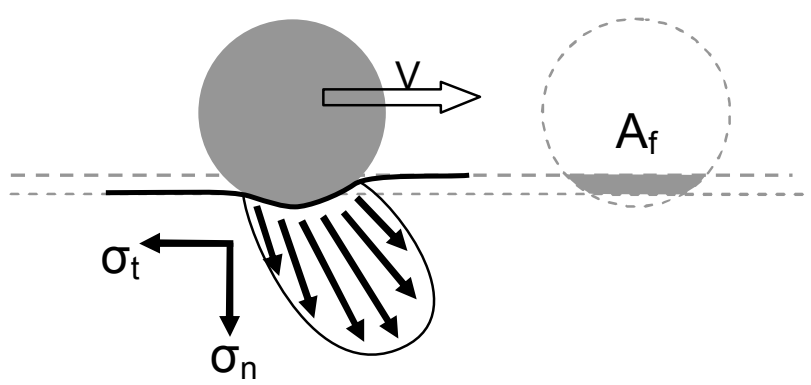

Fig. 2.9 Deformation is produced by ploughing of the harder material through the softer material. The asymmetry of the stress field is characteristic of viscoelastic materials, due to their time-dependent mechanical properties. 
The deformation produced by a rigid asperity ploughing through an elastomer differs from the plastic ploughing due to the viscoelastic behaviour of the elastomers. When a hard asperity ploughs the surface of an elastomer, the deformation of the surface is not completely permanent. After the pressure is released and some time has elapsed, almost all the surface goes back to its original shape. The energy loss in the deformedreformed cycle is called hysteresis, which is characteristic of viscoelastic behaviour [31].

\subsubsection{Factors influencing the sliding friction on elastomers}

The mechanisms of friction are influenced by certain factors involved in the sliding contact between two surfaces and they depend strongly on the material characteristics of the surfaces in contact. This means that the important factors influencing the friction mechanisms are not the same for metals, ceramics or elastomers. A review of the most relevant factors influencing the sliding friction of elastomers is presented below.

\subsubsection{Influence of sliding velocity}

The viscoelastic nature of elastomers, including such phenomena as hysteresis, stress relaxation and creep compliance, explains the influence of velocity under a loading-unloading cycle on the mechanical response of the material. Early observations made by Grosch [33] involved sliding experiments on rubbers using a "flat on flat" geometry, varying the rubber composition and the sliding velocity. The maximum velocity of the experiments was set to $0.88 \mathrm{~cm} / \mathrm{s}$ to limit the amount of frictional heating. Fig. 2.10 shows that the sliding velocity has a large influence on the coefficient of friction. This influence is also dependent on the temperature of the samples, which range from $-15^{\circ} \mathrm{C}$ to $+15^{\circ} \mathrm{C}$. 


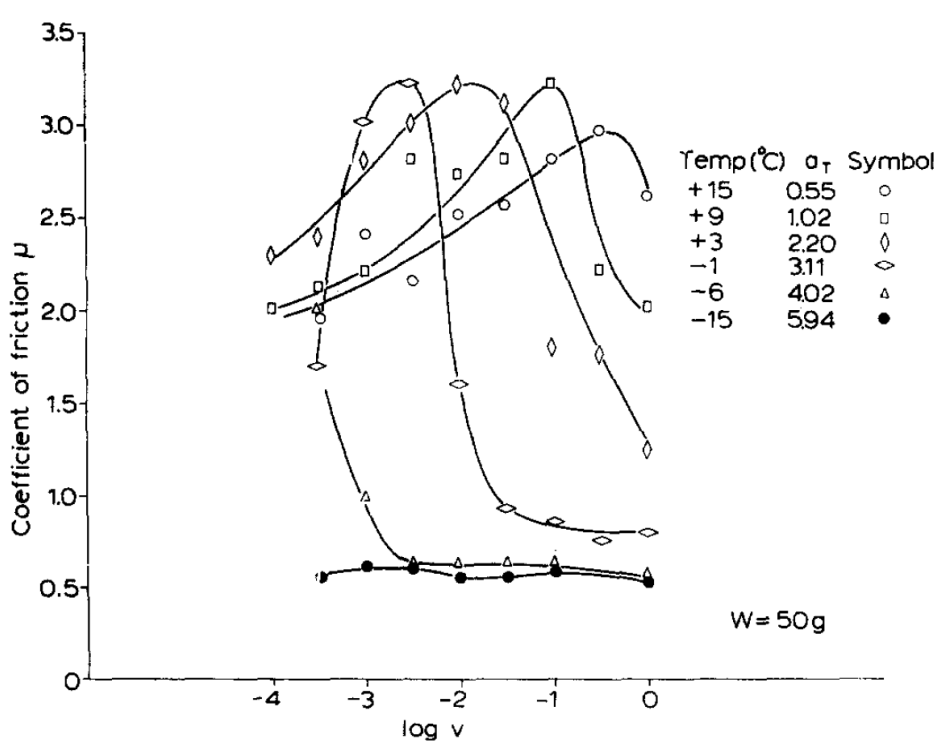

Fig. 2.10: Friction measurements of a glass sphere sliding over a surface of Acrylonitrile-butadiene rubber (NBR) [33]. Results are presented for 6 different temperatures from $-15^{\circ} \mathrm{C}$ to $+15^{\circ} \mathrm{C}$ and the velocity is increased from $0.9 \mu \mathrm{m} / \mathrm{s}$ to $8800 \mu \mathrm{m} / \mathrm{s}$.

Similar experiments were performed by Barquins and Roberts [32], using the same NBR compound and glass counter-surface as Grosch [33]. Their results, in Fig. 2.11, show that the value of the coefficient of friction is independent of the sliding velocity, but depends greatly on the radius of the glass counter surface. The friction coefficient is not affected by the sliding velocity until a certain sliding velocity is reached. It is important to note that these friction experiments were performed at an ambient temperature of $22^{\circ} \mathrm{C}$, which is higher than the friction experiments performed by Grosch [33]. 


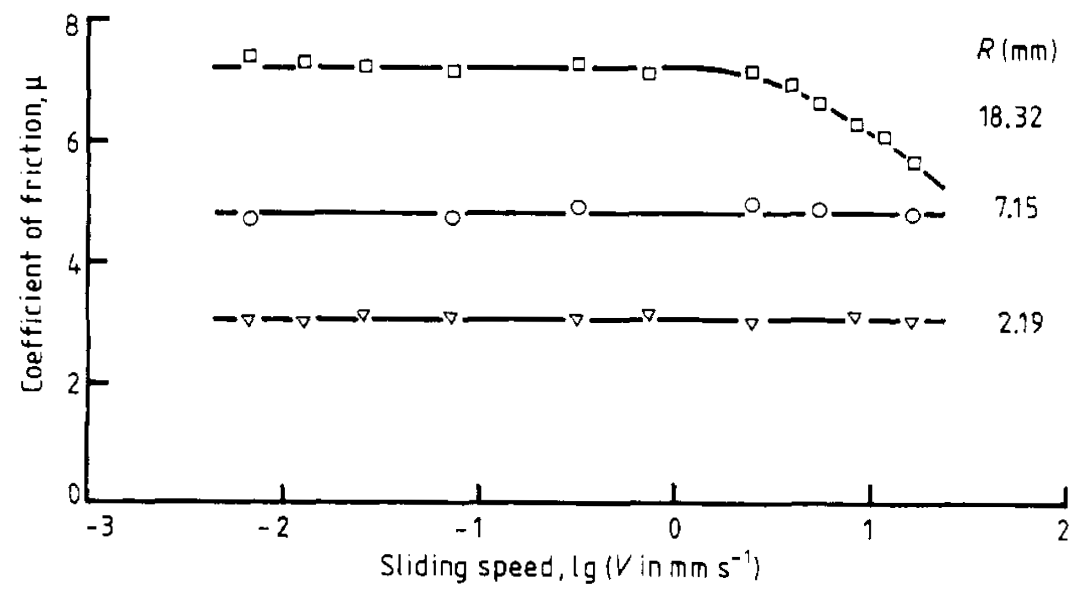

Fig. 2.11 Coefficient of friction depending on sliding speed for NBR sliding against glass lenses of different radius $(R)$. The applied load is $0.05 \mathrm{~N}$ and the temperature is $22^{\circ} \mathrm{C}$.

From the study of Grosch [33] and the study of Barquins and Roberts [32] it can be concluded that the dependence of the coefficient of friction on the sliding velocity is closely related to the ambient temperature during the experiments and the radius of the indenter or the contact pressure.

\subsubsection{Influence of temperature}

As mentioned previously, the dependence of the material properties on the temperature is significant, see Fig. 2.1. This dependence can be summarized as follows: at low temperatures the elastomer reacts as a "hard" material, in its "glassy" region. At higher temperatures, the material passes a transition region in which the static modulus decreases rapidly, until it reaches the elastomeric region. In this state, the static modulus is approximately constant with changing temperatures. By increasing the temperature even further, the elastomer reaches the flow region, where it becomes much softer and is unsuitable for practical use [34].

Considering the dependence of the material properties on the temperature, the experimental results of Grosch could be explained by the fact that at $15^{\circ} \mathrm{C}$ the NBR was in its glassy region and thus showing a very low friction coefficient. As the temperature increases, the NBR sample passes to the transition zone showing lower mechanical properties. This causes an increase of the contact area and as a result the friction coefficient 
increases. It can be seen in Fig. 2.10 that the coefficient of friction in the measurements between $-1{ }^{\circ} \mathrm{C}$ and $+15^{\circ} \mathrm{C}$ were fitted with a bell-shaped curve. At higher temperatures the elastomeric region is reached. Therefore the mechanical properties of the NBR sample are rather constant, showing that the influence of the sliding velocity on the coefficient of friction decreases. The experiments of Barquins and Roberts [32] show that the dependence of the coefficient of friction on the sliding velocity, at $22^{\circ} \mathrm{C}$, is almost non-existent. This can be related to the almost unchanging mechanical properties of the NBR at this temperature, because the material is in its elastomeric region. To know if this hypothesis is valid, it is necessary to know how the mechanical properties of the NBR used by Grosch and by Barquins and Roberts change with the temperature.

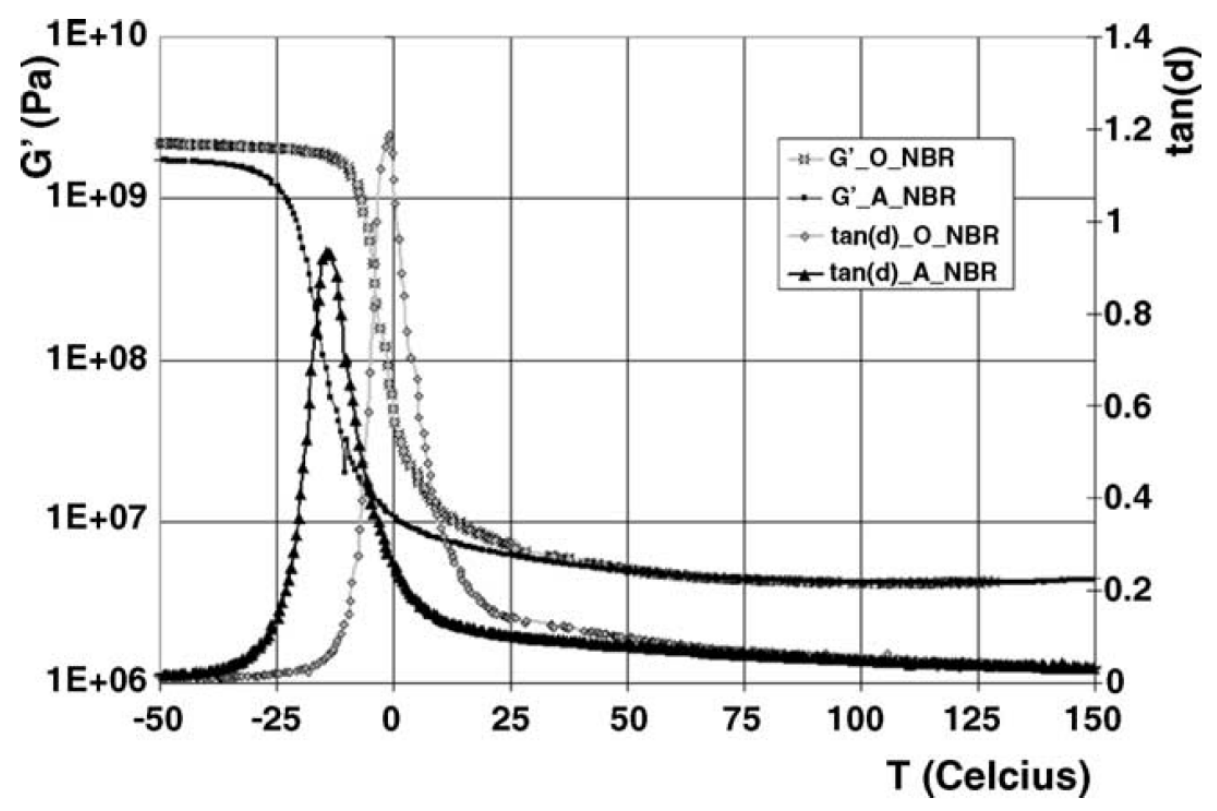

Fig. 2.12 Dynamical shear modulus of two types of NBR, at different temperatures, taken from [45].

The dynamical shear moduli for two different types of NBR are shown in Fig. 2.12. The difference between the two types of NBR is the glass transition temperature $\left(T_{g}\right), T_{g(\text { NBR_A })}=-5^{\circ} \mathrm{C}$ and $T_{g(\text { NBR_O })}=10^{\circ} \mathrm{C}$. If the NBR compound used by Grosch [33] and by Barquins and Roberts [32] follows a similar behaviour as those shown in Fig. 2.12, the dependence of the friction coefficient on the sliding velocity is based on its temperature 
dependency. Therefore friction experiments should be performed at temperatures at which the properties of the material are fairly constant and representative of the problem to be solved.

\subsubsection{Influence of applied load}

Friction experiments on soft elastomers sliding against hard counter surfaces were performed by Roth et al. $[35,36]$ at a sliding velocity of 0.1 $\mathrm{cm} / \mathrm{s}$. These experiments showed that the coefficient of friction decreases with increasing applied load as is found for polymers, see Fig. 2.13.

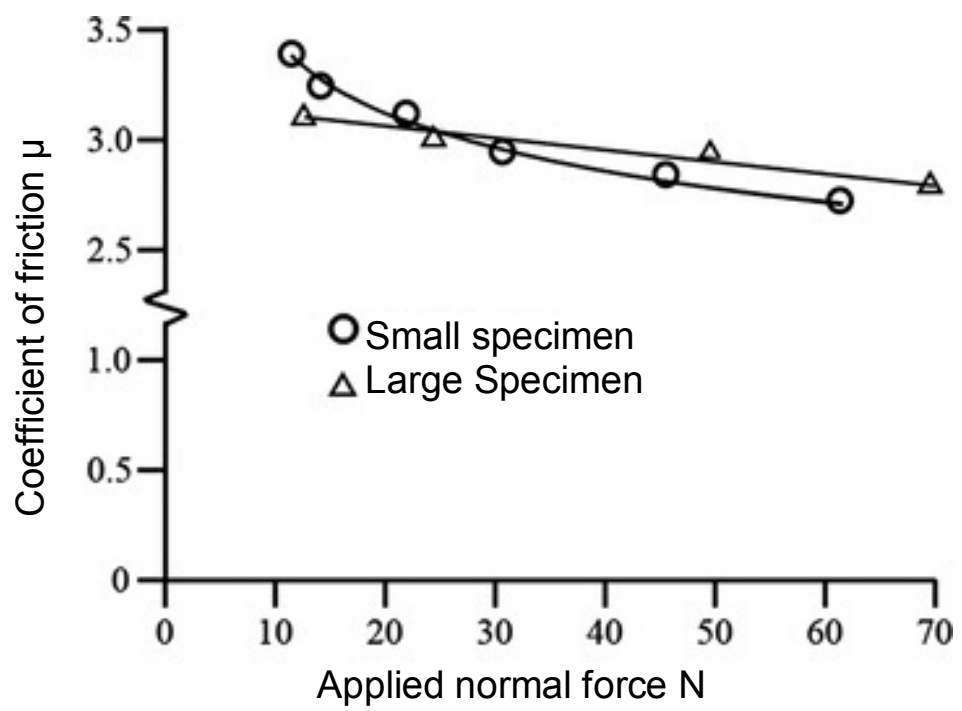

Fig. 2.13: Coefficient of friction for different normal loads [35, 36]. The sliding velocity is $0.1[\mathrm{~cm} / \mathrm{s}]$.

Another observation from the experiments of Roth et al. [35, 36] is that different sizes of indenter lead to different values for the coefficient of friction, concluding that the latter depends on the applied load and the contact area.

\subsubsection{Surface properties and interface}

The adhesive friction mechanism depends greatly on the surface properties of the contacting bodies, as it reflects the energy of interfacial bonding between the surfaces. This energy is related to the nature and density of the interfacial bonds. 
Therefore it is important to characterize the properties of the surfaces in contact. The properties of the surface of a material are not necessarily related to the bulk properties of the material. Previous studies on polymers [38-41] suggested differences, e.g. chemical, between the surface and the bulk. These differences could be caused by the different environments encountered at the surface.

As well as the surface properties affecting the friction, friction can also affect the surface properties of the contacting bodies and its interface, in the same way as mechanical deformation changes the mechanical behaviour of a work hardening material. An example of how mechanical deformation of an elastomer affects its mechanical properties is the Mullins effect, observed in repeated stress-strain measurements [9].

Friction also influences the surface properties of polymers. Toney et al. [42] found that rubbing a polyamide film causes alignment of the polymer molecules near the surface. This alignment causes significant differences between the properties of the surface and the bulk. PTFE (Polytetrafluoroethylene or Teflon) and PE (Polyethylene) are also known to show molecular orientation at the surfaces as a response to friction.

Karger-Kocsis et al. [43] studied the tribological behaviour of EPDM with different concentrations of carbon black. Differences found in the coefficient of friction and wear were attributed to tribo-chemical reactions and thermooxidative degradation of the EPDM. Another tribological study of EPDM with different concentrations of carbon black was performed by Martinez et al. [44]. The concentrations of carbon black used in this study are similar to the concentrations used by Karger-Kocsis et al. [43]. This study revealed that different concentrations of carbon black have little effect on the chemical properties of the EPDM in the unworn state, but have a high influence on the chemical properties of the EPDM after sliding by a steel indenter. The differences in the chemical composition of the worn areas were found to be related to the mechanical rupture of the bonds. This indicates that the difference between the tribological behaviour of the EPDM samples originates in the modification of the sliding interface for different carbon black contents.

Furthermore, friction studies identify the creation of an interfacial layer as the cause of the variation in the tribological behaviour. Thomine et al. [45] compared two different elastomers by making sliding friction experiments. They found that one of the rubbers underwent thermo-oxidation in the surface, which explained the reduction of the sliding friction in comparison with the other elastomer. Deladi [46] found an interfacial layer formed in the contact of an elastomer with a metal sheet. This layer also influences the 
tribological behaviour. Analysis performed using an SEM indicated the thickness of this layer to be between 0.1 and $0.8 \mu \mathrm{m}$.

In conclusion, it is important to characterize the surface properties. In the case where sliding friction causes modifications in the surface properties, it is also necessary to estimate the properties of this interfacial layer, as it plays an important role in the friction due to adhesion.

\subsection{Summary}

To describe the sliding friction of a tribological system it is first necessary to describe the contact between the contacting surfaces. There are contact models available in literature that allow the radius of the contact and the indentation depth to be calculated under certain restrictions. The Hertz contact model assumes elastic and isotropic materials without adhesive forces between the surfaces. A variety of elastic models considering adhesion at different levels of elasticity and loads are also available. Elastic contact models considering different levels of anisotropy [15-20] and viscoelastic isotropic contact models [11-14] are available as well. However, a contact model that is able to integrate anisotropic behaviour, viscoelastic behaviour and adhesional forces between the surfaces in contact is not available in literature.

The addition of fibres to the elastomeric matrix indicates that the composite material will behave anisotropically. The viscoelastic character of the elastomer indicates that the composite material will also have viscoelastic behaviour. The presence or absence of adhesional forces between the surfaces in contact depends on the elasticity parameter and the load parameter. Therefore the influence of adhesional forces can be determined only after characterizing the tribological system. Hence after characterizing the tribological system and determining the importance of adhesional forces in the contact, the corresponding contact model must be developed.

The friction between two surfaces in dry contact is described by two mechanisms: adhesion and deformation. The importance of each friction mechanism in systems involving elastomers depends greatly on the specific tribological system in study. Consequently, after characterizing the tribological system it will be possible to estimate the most relevant mechanism of friction. For this, it is necessary to quantify parameters such as sliding velocity of the contacting bodies, normal load applied in the contact and ambient temperature at which the contact occurs. 


\section{Chapter 3 The tribological system in this thesis}

One of the potential practical applications of this research is to develop automotive tyres with improved traction and minimised rolling resistance. These properties may be obtained by reinforcing the elastomer with fibres. However, the potential tribological benefits of fibre reinforced elastomers are not restricted to automotive tyres. Therefore the research presented in this thesis aims to provide a more general description of the contact and friction behaviour of fibre reinforced elastomeric materials. Among the elastomer-filler material systems studied is EPDM filled with Twaron $®$ aramid fibres supplied by Teijin Aramid B.V.

This chapter focuses on the main considerations involving the presented tribological system. In Appendix A the tribological system in study is introduced, the contact and friction behaviour of a fibre reinforced elastomer against a rigid surface in a tyre road contact configuration. In this configuration the rubber from the tyre is in sliding motion with the rigid pavement. The rubber is modelled as an anisotropic viscoelastic surface and the pavement is modelled as a series of spherical rigid indenters. The sliding friction of tribo-systems in which one of the contacting bodies is an elastomer was discussed in Chapter 2. The behaviour of the contact area and the influencing parameters were presented, as well as the friction mechanisms involved in sliding friction and the factors that influence the friction behaviour of elastomers. It was concluded that in order to characterize the friction behaviour, it was necessary to study the behaviour of the contact area.

To characterize the contact area, details related to the materials in contact, such as viscoelastic and anisotropic material behaviour, must be determined, as well as details about the interaction between the surfaces in contact, such as contact pressure and adhesion forces between the surfaces.

To characterize the friction behaviour it is necessary to determine the contact and friction conditions that represent the system in study, such as sliding velocity, applied load and environmental conditions. Based on this, the friction mechanism that has the stronger influence on the sliding friction of the present tribo-system can be determined. 


\subsection{Material behaviour of the contacting bodies}

In the present tribological system the tyre road contact is studied as a single asperity contact situation, in which the roughness of the pavement is represented by a sphere, with an elastic modulus much higher than the elastic modulus of the elastomer. The fibre reinforced elastomer exhibits two types of "special" material behaviour: anisotropy and viscoelasticity. Considerations regarding both kinds of behaviour follow.

\subsubsection{Anisotropic behaviour}

Adding fibres to the elastomer will generate some anisotropy in the material. In this thesis, a few assumptions are made: the fibres are homogeneously distributed in the elastomeric matrix; they have a preferred orientation within the matrix; the composite has three perpendicular symmetry planes which means that the material will behave orthotropically; and the contact between the two surfaces will occur in one of the principal planes of the orthotropic material, see the examples in Fig. 3.1. The "shape" of the compliance and stiffness matrix is therefore known.
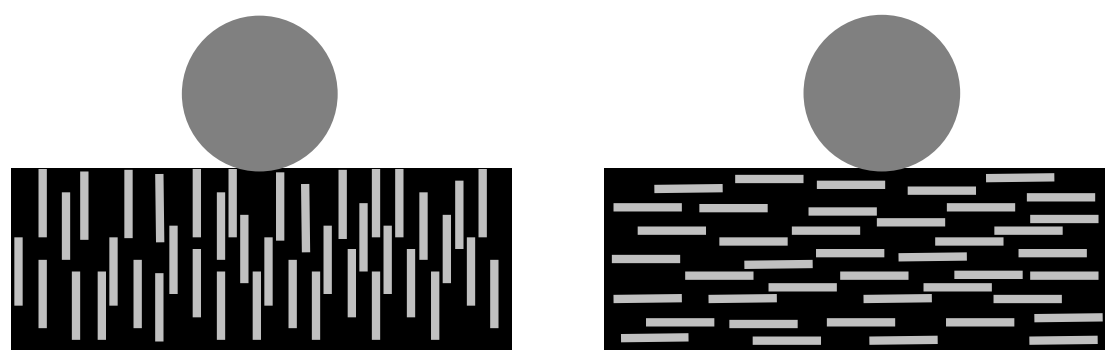

Fig. 3.1 Schematic representation of the orientation of fibres in the elastomer. On the left the fibres are oriented along the indentation direction; on the right the fibres are oriented in the plane of indentation.

To be able to model the material as a continuum, the thickness of the fibres must be smaller than the contact area between the surfaces in contact. The length of the aramid fibres is approximately $3 \mathrm{~mm}$ after mixing and their diameter is $10-12 \mu \mathrm{m}$. As the diameter is of the order of micrometers and the contact area is of the order of millimetres, the presence of the fibres is almost negligible in the contact area and the elastomer composite can be modelled as a continuum. 
The tribological system in this thesis

\subsubsection{Viscoelastic behaviour}

Polymers are usually modelled as linear viscoelastic materials. Elastomers are hyperelastic materials meaning that they can be modelled as linear viscoelastic materials only at strains lower than 100\% [47]. The word "linear", regarding viscoelastic theory, refers to a linear time dependency between the stress and strain. This results in a correlation between the dynamic and the transient properties of the material [47-48]. The dynamic properties include the storage and the loss modulus, and the transient properties include the stress relaxation and the creep compliance. These properties and their correlations can be described by mechanical models composed of springs and dashpots [47-48]. The springs represent the elastic behaviour and the dashpots represent the viscous behaviour of the material (see the example in Fig. 3.4).

In this thesis, the model tribo-system in study is the tyre-road contact with strains lower than 10\% [49]. Therefore the viscoelastic behaviour can be characterized by linear viscoelasticity. It is shown below that it is possible to use the linear theory of viscoelasticity to model the studied elastomers and fibre reinforced elastomers. Dynamical measurements were performed and the results show a good fit with functions from the linear theory of viscoelasticity.

The shear storage modulus and shear loss modulus of Ethylene Propylene Diene Rubber, EPDM, were measured in a Dynamic Mechanical Analysis (DMA) in shear using a Metravib Viscoanalyser DMA+150, in frequency sweep mode (from 0 to $200 \mathrm{~Hz}$ ), at $0.5 \%$ strain without pre-strain and at ambient temperature. This device and the specimen configuration are depicted in Fig. 3.2. 

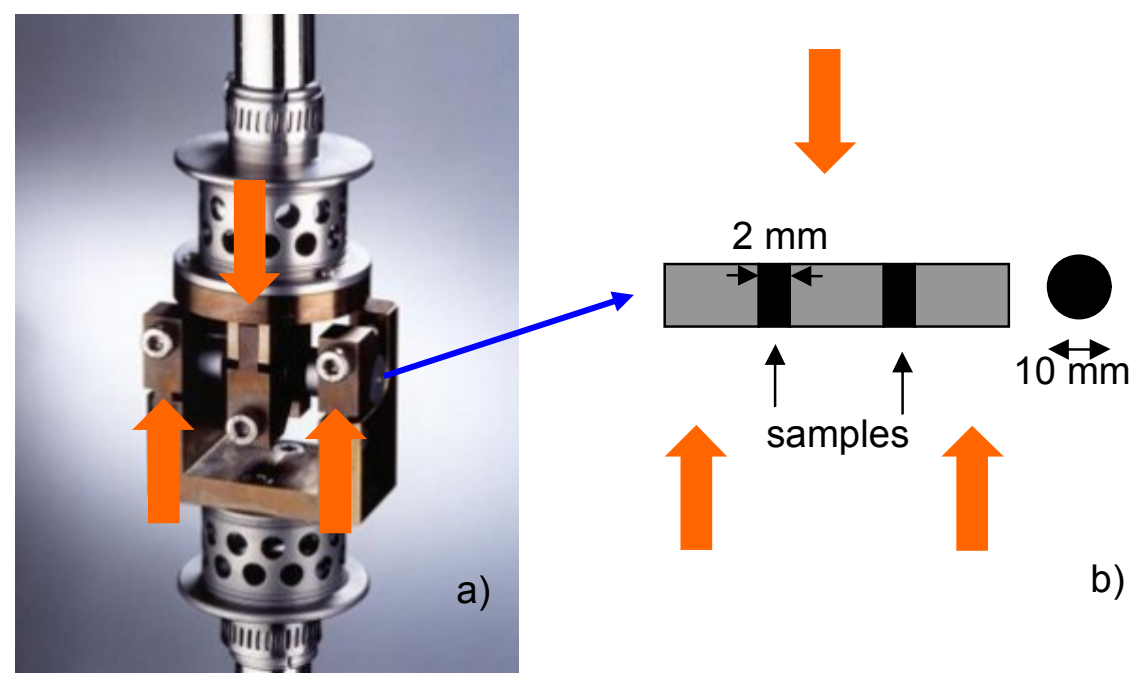

Fig. 3.2: Metravib Viscoanalyser DMA+150 with specimen holder to study shear storage modulus and shear loss modulus (a) and the specimen configuration (b).

The specimens shown in black in Fig. 3.2, b) were cut from elastomer sheets of $2 \mathrm{~mm}$ thickness, in a circular shape of $10 \mathrm{~mm}$ diameter and glued to the holder (in grey). By shearing the specimen with $0.5 \%$ strain at increasing frequency, the shear storage and shear loss modulus were determined.

Two types of elastomers were used, EPDM without fibres and fibre reinforced EPDM with a concentration of 5 parts per hundred parts of rubber (phr). The fibres used are Twaron $®$ aramid fibres. The results from the DMA measurements, for the unreinforced EPDM are shown in Fig. 3.3. 
a)

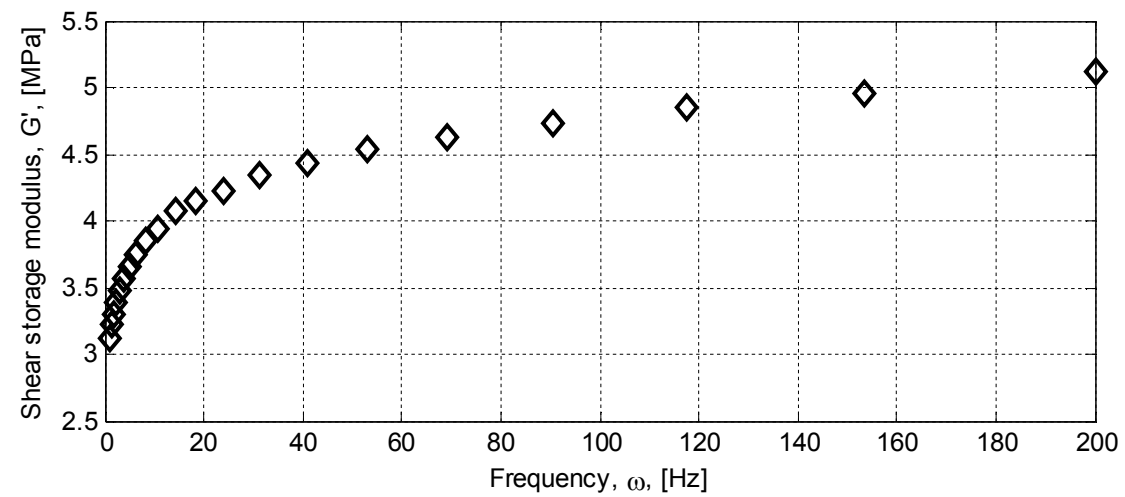

b)

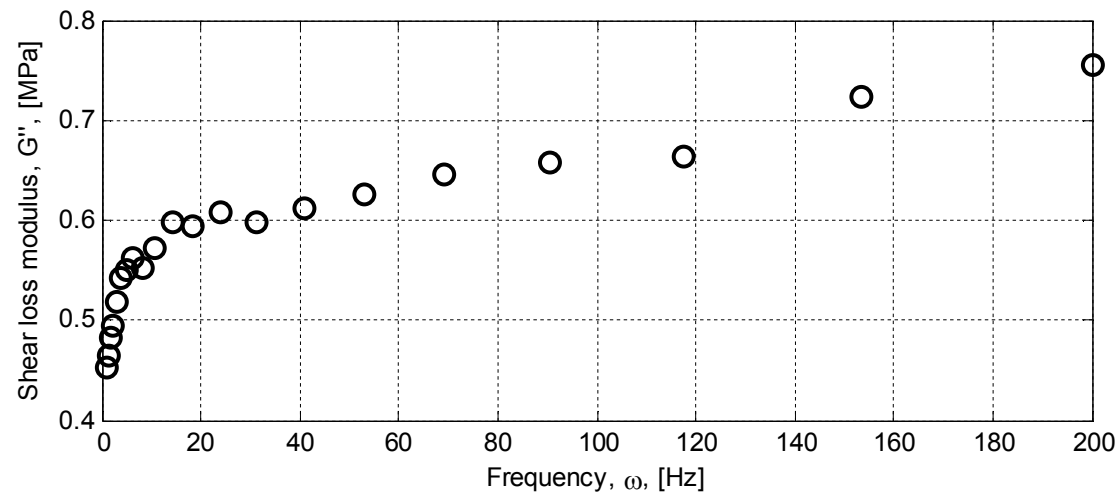

Fig. 3.3: a) shows the shear storage, G', b) shows the shear loss, G', modulus of EPDM, measured in frequency sweep mode.

To model this viscoelastic material behaviour according to the linear theory of viscoelasticity, the shear storage modulus and shear loss modulus of the EPDM can be fitted with a Prony series representation of springs and dashpots from Maxwell's model (Fig. 3.4) in the form of Eq. 3.1 and Eq. 3.2 [48]. 


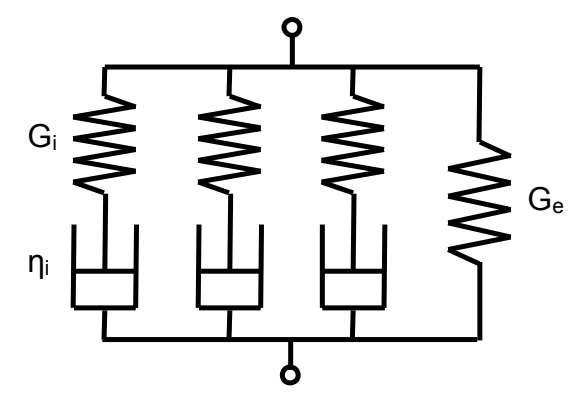

Fig. 3.4: Schematic representation of the Prony series [48].

The storage modulus is given by:

$$
G^{\prime}(\omega)=G_{e}+\sum_{i=1}^{m} G_{i} \frac{\omega^{2} \tau_{i}^{2}}{\omega^{2} \tau_{i}^{2}+1}
$$

and the loss modulus by:

$$
G^{\prime \prime}(\omega)=\sum_{i=1}^{m} G_{i} \cdot \frac{\left(\omega \tau_{i}\right)}{1+\left(\omega \tau_{i}\right)^{2}}
$$

where $G_{e}$ represents the equilibrium modulus, $G_{i}$ the relaxation strength and $\tau_{i}$ the relaxation times. The relaxation times $\tau_{i}$, are related to $\eta_{i}$ as $\eta_{i} / G_{i}$. Fig. 3.5 shows for both EPDM and fibre reinforced EPDM the shear storage and shear loss modulus fitted to a Prony series in which $m=3$. 
The tribological system in this thesis

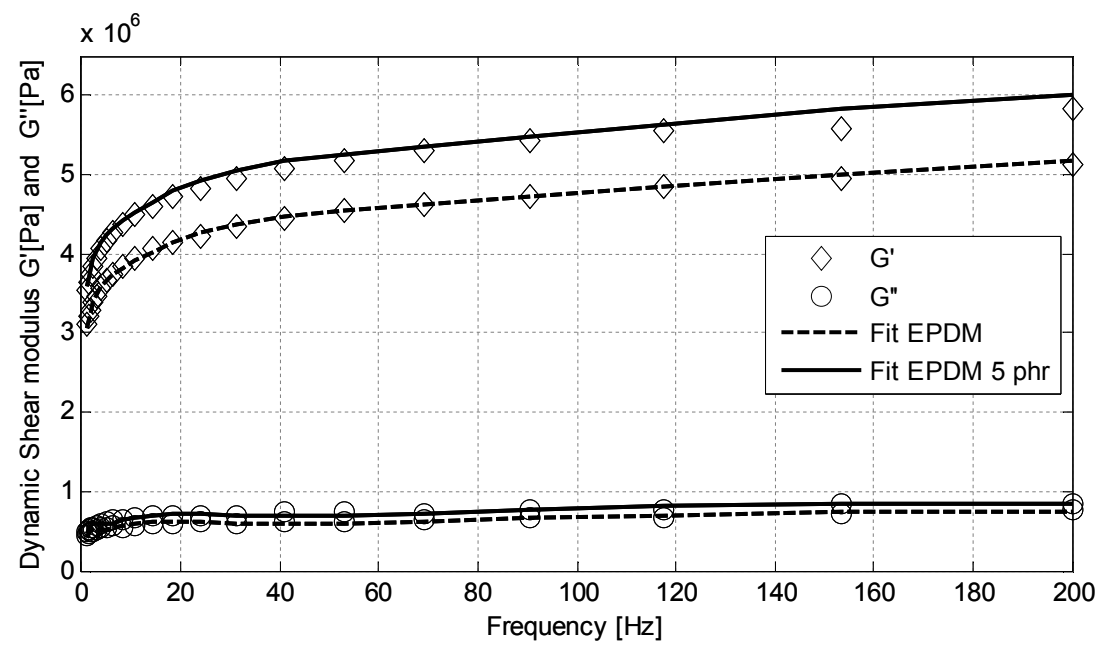

Fig. 3.5: The shear storage and shear loss modulus of the EPDM and fibre reinforced EPDM, both fitted according to the Prony series $(m=3)$.

The parameters of the EPDM and fibre reinforced EPDM with $5 \mathrm{phr}$ of aramid fibres are summarized in Table 3.1.

Table 3.1: Prony series parameters.

\begin{tabular}{ccc}
\hline Parameter & EPDM & EPDM 5 phr \\
\hline $\mathrm{G}_{\mathrm{e}}[\mathrm{MPa}]$ & 2.86 & 3.35 \\
$\mathrm{G}_{1}[\mathrm{MPa}]$ & 0.83 & 0.91 \\
$\mathrm{G}_{2}[\mathrm{MPa}]$ & 0.84 & 0.97 \\
$\mathrm{G}_{3}[\mathrm{MPa}]$ & 1.31 & 1.48 \\
$\tau_{1}[\mathrm{~s}]$ & 0.560 & 0.574 \\
$\tau_{2}[\mathrm{~s}]$ & 0.0581 & 0.0582 \\
$\tau_{3}[\mathrm{~s}]$ & 0.00499 & 0.00524 \\
\hline
\end{tabular}

As can be seen in Fig. 3.5, the viscoelastic behaviour of the elastomer and the fibre reinforced elastomer are very well described by this kind of time function. Therefore the viscoelastic behaviour of the material will be modelled using the linear viscoelastic theory, which allows using the elastic - viscoelastic correspondence principle and the correlations of dynamic and transient properties. 


\subsection{Interaction between the contacting surfaces}

In a tribological system, the interaction between the contacting bodies is closely related to the contact and friction behaviour of the system. Therefore it is important to determine these conditions that represent the tribo-system. This representation allows the contact and friction behaviour of the studied system to be emulated in a similar small-scale tribological system under controlled laboratory conditions. Among the factors that determine the interaction between the contacting surfaces in the present tribo-system are the sliding velocity, the applied load (contact pressure) between the surfaces, the existence of adhesional forces between the surfaces and the environmental conditions such as temperature.

\subsubsection{Sliding velocity of the contacting surfaces}

The sliding conditions in the model-tribosystem will be chosen so that they are similar to those between a tyre and the road. A typical tyre of a passenger car has a radius of $R \approx 30 \mathrm{~cm}$ and the contact patch between the road and the tyre has a length of approximately $L \approx 10 \mathrm{~cm}$. From this, the deformation of the tyre and, thus, the sliding conditions within the contact can be calculated. 


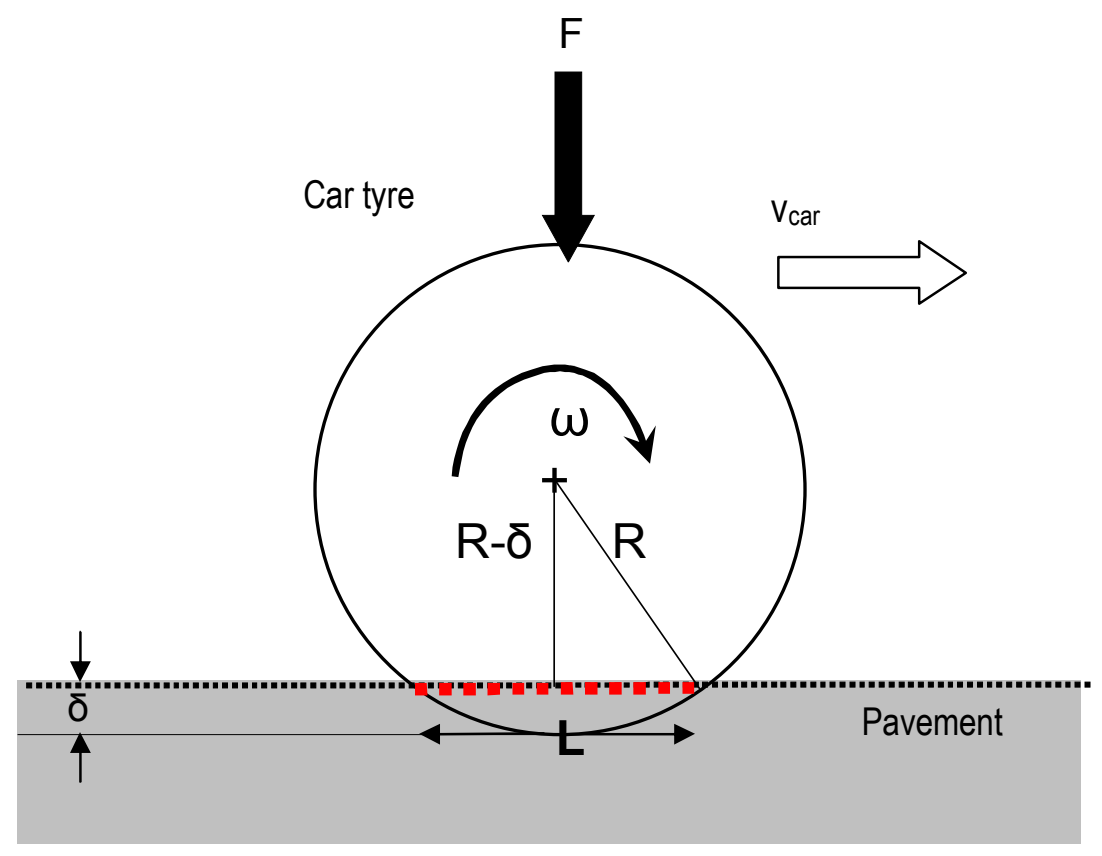

Fig. 3.6 Schematic of a car tyre in contact with the pavement, and the contact patch of length $L$.

The normal deformation of the car tyre can be calculated from trigonometry relations between the radius of the car tyre and the length of the contact patch.

$$
\delta=R-\sqrt{R^{2}-\left(\frac{L}{2}\right)^{2}}=0.004 \mathrm{~m}
$$

At steady state driving conditions with a constant speed of $\mathrm{v}_{\text {car }}=20 \mathrm{~m} / \mathrm{s}$ the rotational velocity of the tyre is given by Eq. 3.4. This value is in agreement with other studies of the tyre road contact [50].

$$
\omega=\frac{\mathrm{v}_{\text {car }}}{R} \approx 70 \frac{\mathrm{rad}}{\mathrm{s}} \Rightarrow 11 \mathrm{~Hz}
$$

The difference in velocity between the tyre and the road is given by:

$$
\Delta \mathrm{v}=\omega \delta \approx 0.25 \frac{\mathrm{m}}{\mathrm{s}}
$$


Therefore the sliding velocity of the tribological system is of the order of $\mathrm{v}=0.2 \mathrm{~m} / \mathrm{s}$. To study the influence of the sliding velocity on the present tribological system, sliding friction experiments were performed using a pinon-disk setup, for more details see Appendix D, with a constant normal load of $F_{N}=2 \mathrm{~N}$, a radius of the ball $\mathrm{R}=12.5 \mathrm{~mm}$ at different sliding velocities. The results, see Fig. 3.7, show only a small influence of the sliding velocity on the friction coefficient. This means that slight differences of the sliding velocity in the tribo-system will not affect the frictional behaviour significantly.

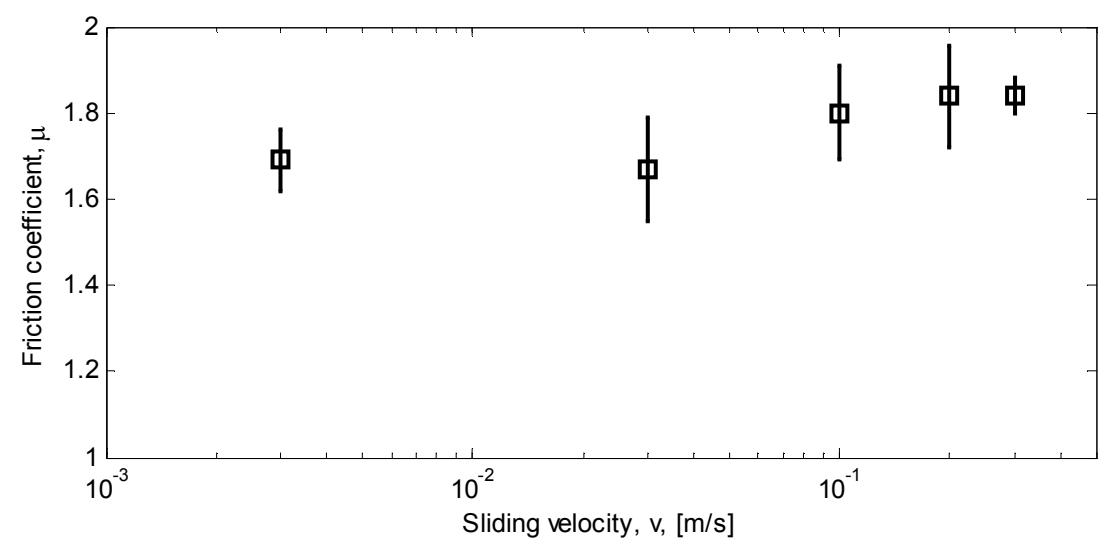

Fig. 3.7 Influence of sliding velocity on the coefficient of friction, steel spherical indenter sliding against EPDM.

\subsubsection{Applied load between the bodies in contact}

The typical contact pressures of the contact patch between a passenger car tyre and the road surface are given by the car tyre pressure and are approximately $p_{m}=0.3 \mathrm{MPa}$. To reproduce the contact conditions in a laboratory setup, the contact theory of Hertz is used. The equation of maximum contact pressure is used [10].

$$
p_{0}=\left(\frac{6 F_{N} E_{*}^{2}}{\pi^{3} R_{*}^{2}}\right)^{1 / 3}
$$

The maximum contact pressure is defined by the normal force $F_{N}$, the equivalent elastic modulus of the elastomer and the pavement $E_{\star}$ and the equivalent radius $R_{*}$. The relation between the maximum, $p_{0}$, and the mean contact pressure is given by $p_{0}=3 p_{m} / 2=0.45 \mathrm{MPa}$. 
The tribological system in this thesis

To calculate the normal load representative of the contact pressure of a tyre-road contact, the material properties of an Ethylene Propylene Diene Rubber (EPDM) are considered as an elastomer model system. The elastic modulus of EPDM, i.e. $E=4.39 \mathrm{MPa}$, and the Poisson's ratio is $v=0.49$ (valid for incompressible materials). The equivalent elastic modulus is $E_{*}=5.78 \mathrm{MPa}$. For the applied normal force $F_{N}=2 \mathrm{~N}$ and different values for the radius of the indenter, the maximum contact pressure is given in Table 3.2.

Table 3.2: Maximum contact pressure for different radii of the indenter at $F_{N}=2 \mathrm{~N}$.

\begin{tabular}{cccc}
\hline Radius & $12.5 \mathrm{~mm}$ & $5 \mathrm{~mm}$ & $2 \mathrm{~mm}$ \\
\hline$p_{0}[\mathrm{MPa}]$ & 0.44 & 0.81 & 1.44 \\
\hline
\end{tabular}

This means that under the given conditions, an indenter of $12.5 \mathrm{~mm}$ radius will cause a similar contact pressure as occurs in the contact patch of the tyre. Other indenters have been included in the study to account for pressure variations, e.g. as the result of roughness in the pavement.

\subsubsection{Adhesional forces in the contact}

To study the adhesional forces present in the contact, the load $(\bar{P})$ and elasticity $(\lambda)$ parameters [30] corresponding to the tyre road tribo-system were calculated from Eq. 2.9 and Eq. 2.10. This requires the contact parameters such as the normal load of the contact and the radius of the indenter, material parameters such as the equivalent elasticity modulus and surface parameters such as the surface tension between the materials in contact. As determined before, the contact parameters of the tribo-system are a normal load of $F_{N}=2 \mathrm{~N}$. Three different radii of the indenter are considered: $R=12.5, R=5$ and $R=2 \mathrm{~mm}$. The material parameter is an equivalent elastic modulus of $E^{*}=5.78 \mathrm{MPa}$. The work of adhesion between the contacting materials was measured by performing contact angle measurements; this is explained further in Appendix $E$. The surface tension between EPDM and steel is $W=70.22 \mathrm{~mJ} / \mathrm{m}^{2}$ and the equilibrium separation between atomic planes is given in literature [51], $\mathrm{z}_{0}=0.3 \mathrm{~nm}$. 
Table 3.3: Load and Elasticity parameters, depending on the radius of the indenter.

\begin{tabular}{cccc}
\hline Indenter radius & $\mathrm{R}_{1}=12.5 \mathrm{~mm}$ & $\mathrm{R}_{2}=5 \mathrm{~mm}$ & $\mathrm{R}_{3}=2 \mathrm{~mm}$ \\
\hline $\bar{P}$ & $0.7310^{3}$ & $1.8110^{3}$ & $4.5310^{3}$ \\
$\lambda$ & $4.7410^{3}$ & $3.4910^{3}$ & $2.5710^{3}$ \\
\hline
\end{tabular}

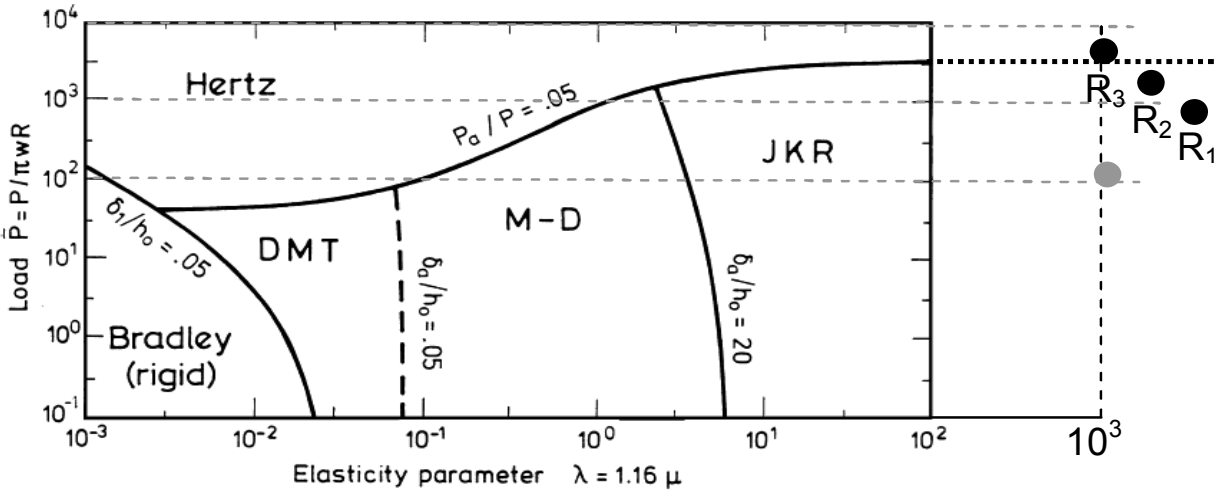

Fig. 3.8: Adhesion map, made by Greenwood and Johnson [30]. The black dots show the position of the studied contact in the map and the grey dot shows the position of Vorvolakos and Chaudhury [26] contact.

Placing the parameters from Table 3.3 into the adhesion map, Fig. 3.8, two suitable contact models were obtained. The contact suitable to be calculated using the Hertz model is the indenter of small radius while the JKR model is used for the indenter of bigger radius. These results are in accordance with results by Greenwood and Johnson [30], who established that in elastomeric materials the elasticity parameter is higher than 100.

The contact in study is compared with results from Vorvolakos [26], see Fig. 2.7, and the position of their contact in the adhesion map. In the present study the position of the contact in the adhesion map is much closer to the Hertz model, and the sliding velocities are much higher than the ones showed in Fig. 2.7. This indicates that there is only a limited influence of adhesion on the contact area and it will therefore be neglected.

\subsubsection{Operating temperature}

The tensile storage and loss modulus of EPDM were measured by Dynamic Mechanical Analysis using a Metravib Viscoanalyser DMA+150 in a temperature sweep mode, without pre strain, at a frequency of $10 \mathrm{~Hz}$. The temperature ranged from $-80^{\circ} \mathrm{C}$ to $+80^{\circ} \mathrm{C}$. Fig. 3.9 shows that at very 
low temperatures $\left(T<-20^{\circ} \mathrm{C}\right)$ the storage and loss modulus both vary greatly, but from $20^{\circ} \mathrm{C}$ and higher, both storage and loss modulus do not vary quite as much. Therefore in the tribo-system a representative temperature of $23^{\circ} \mathrm{C}$ was chosen. This temperature is within the elastomeric region of the material.

a)

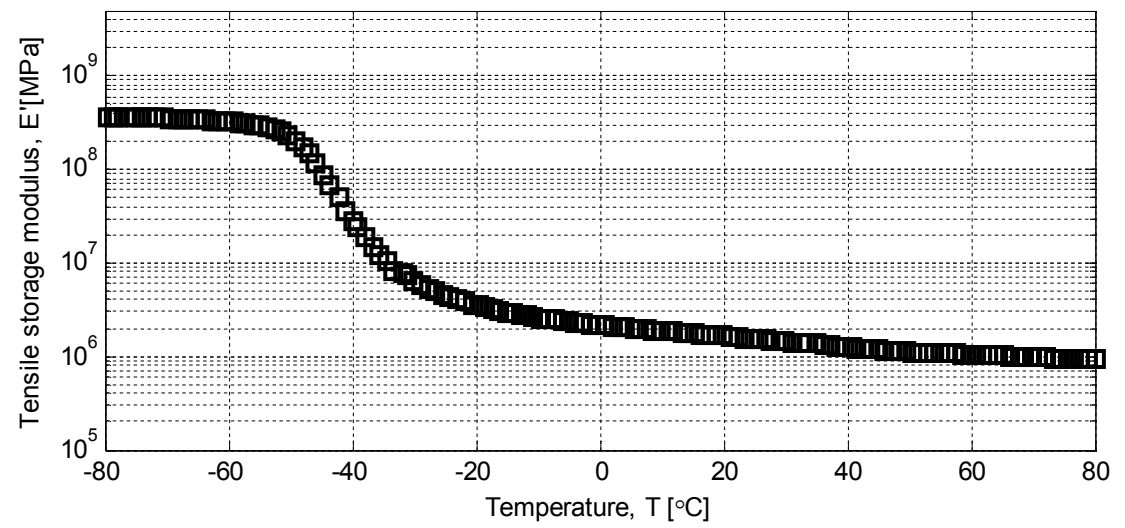

b)

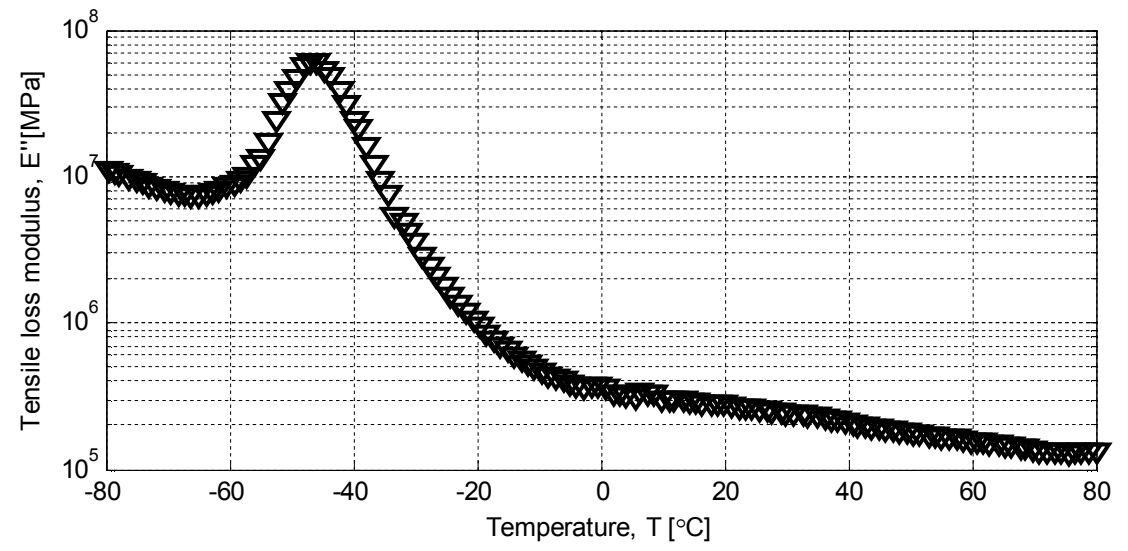

Fig. 3.9: In a) the tensile storage modulus and in b) the tensile loss modulus of EPDM, both measured in temperature sweep mode.

To estimate the amount of frictional heating, pin-on-disk experiments were performed at a sliding velocity of $0.2 \mathrm{~m} / \mathrm{s}$ and a controlled temperature of $23^{\circ} \mathrm{C}$. The temperature in the contact patch was measured by placing a thermocouple at the contact exit. Under these sliding conditions, an increase of temperature of less than $5^{\circ} \mathrm{C}$ was observed. As the contact occurs in the elastomeric region $\left(23^{\circ} \mathrm{C}\right)$, the material properties remain very similar. Therefore in this thesis the effect of frictional heating is ignored. 


\subsection{Mechanisms of sliding friction}

As mentioned previously, sliding friction has its origin in two macroscopic processes. One of them is deformation, which has its origin in harder asperities ploughing through the softer material. The other mechanism is adhesion and has a physical origin in shearing the surface layers/interfacial layer between the opposing surfaces.

In order to estimate the importance of these two mechanisms under the present tribological conditions, sliding experiments were performed on a pin-on-disk setup (details of the setup can be seen in Appendix D). The indenter, pin, is rigid and the disc is the elastomer. This configuration enables to observe both mechanisms of friction, adhesion and deformation. These results are followed by experiments isolating the deformation mechanism of friction.

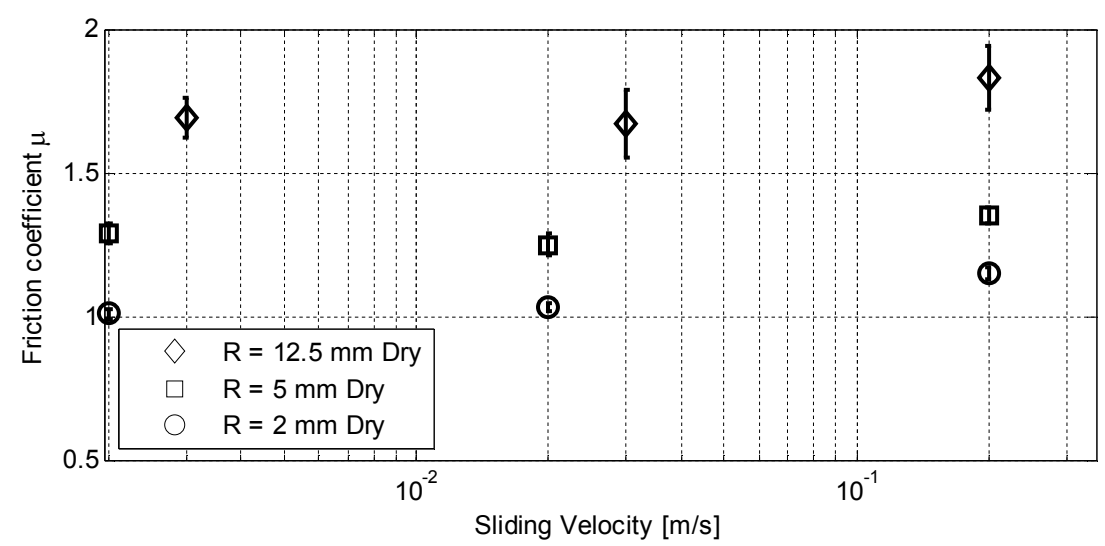

Fig. 3.10: The coefficient of friction between the EPDM and a spherical indenter in dry contact conditions (different indenter radii and sliding velocity).

The normal load was kept constant, at $F_{N}=2 \mathrm{~N}$, while varying the sliding velocity, between $v=0.002-0.2 \mathrm{~m} / \mathrm{s}$. Three indenters with different radii were used, $R=12.5 \mathrm{~mm}, R=5 \mathrm{~mm}$ and $R=2 \mathrm{~mm}$. Fig. 3.10 shows the results of sliding friction experiments using an EPDM sample with properties summarized in Appendix C. It can be seen that the coefficient of friction for an indenter with the bigger radius is higher than for the indenter with the small radius, and that the sliding speed has little effect on the coefficient of friction for the three different indenters. The coefficient of friction in a dry contact, at different radius of indenter and at different sliding velocities, is higher than one. 
The tribological system in this thesis

To isolate the deformation mechanism of friction, a similar experiment was conducted but adding a thin boundary lubricant layer between the specimens. By having the contact lubricated, the influence of adhesion is minimized and the effect of the ploughing would be dominant. The new contact, being lubricated, may function in one of the three lubrication regimes: boundary lubrication $\mathrm{BL}$ (at low sliding velocities, in which the load is carried by asperities), elasto-hydrodynamic lubrication $\mathrm{EHL}$ (where the load is carried by the lubricant due to pressure build up at high sliding velocities) and mixed lubrication ML (at intermediate velocities, where the normal load is carried by both the fluid and the asperities). To ensure that the ploughing effect is dominant (meaning that the entire load is carried by the contacting asperities) the contact must function under the boundary lubrication regime. The friction in the system is controlled by shearing both the boundary layer and surfaces of the bodies in contact. Hence the influence of the adhesion mechanism is excluded.

An oil of low viscosity, EZL $799 \eta=0.074 \mathrm{~Pa} \mathrm{~s}$, was used to cover the surface of the rubber disk. The thickness of the lubricant layer on the EPDM surface was measured by weighing the specimen before and after uniformly applying the oil. The thickness was approximately $1 \mu \mathrm{m}$, placing the contact in the boundary lubrication regime because the roughness of the surfaces in contact, at a cut-off length of $800 \mu \mathrm{m}$, is of the order of 2.9 $\mu \mathrm{m}$.

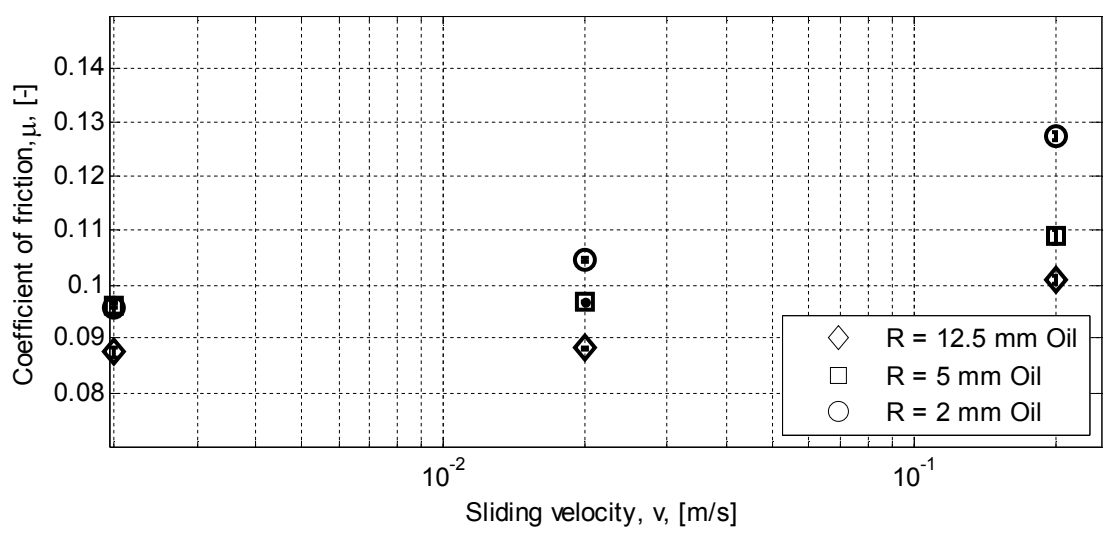

Fig. 3.11: The coefficient of friction between the EPDM and a spherical indenter in lubricated contact conditions.

The results of the experiments are shown in Fig. 3.11. In the presence of the lubricant layer, the coefficient of friction reduces significantly. The 
results are in agreement with other studies [25] (Fig. 3.12), where the coefficient of friction at different sliding velocities (lower than the ones in this thesis) shows a significant decrease after applying a lubricant in the contact interface. This effect is more pronounced at low sliding velocities.

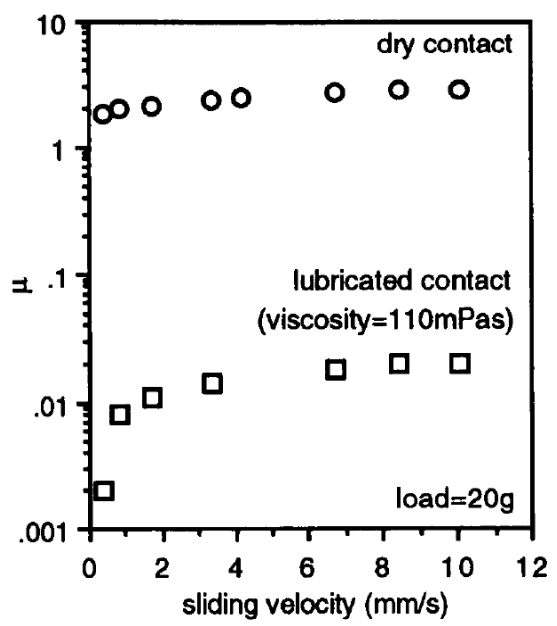

Fig. 3.12: The coefficient of friction obtained in dry and lubricated sliding contact between an elastomer and a concave lens [25].

From these experimental results, it is concluded that the most important friction mechanism in the present tribological system, i.e. under dry sliding conditions, is adhesion and that deformation (ploughing) is responsible for only a small percentage of the friction and therefore will be neglected.

To calculate the friction force due to adhesion, the expression, $F_{a}=\tau A$, of Bowden and Tabor will be used. It is therefore important to predict the contact area generated when the surfaces are in contact during sliding as well as the shear strength of the surface, to calculate the friction force.

\subsection{Summary}

To characterize the contact area, details regarding the material behaviour of the contacting surfaces, such as viscoelasticity and anisotropy, as well as the interaction between them, such as attractive forces between the contacting surfaces, must be determined.

Regarding the viscoelastic behaviour of the elastomers in study, it was concluded that due to the small strains in the contact patch it is possible to 
The tribological system in this thesis

model the elastomers using the linear theory of viscoelasticity. This was verified by performing DMA measurements; the results fit the equations from linear theory of viscoelasticity. The contact of the material without fibres can be described by the contact models for viscoelastic materials available in literature [11-14].

The fibres in the matrix will have diameters much smaller than the contact area, allowing the anisotropic material to be considered as a continuum. The fibres will be distributed homogeneously in the elastomer, with a preferred orientation forming an orthotropic material. The principal axes of the orthotropic material are in accordance with the main axis of the contact. The reference coordinate system has one axis in the direction of indentation and two perpendicular axes in the plane of indentation. In this way the stiffness matrix will have a known form and the elastic contact area can be calculated from the elastic anisotropic contact models [16-19].

The adhesion map was used to determine the importance of adhesion in the interaction between the contacting surfaces. To calculate the load parameter and elastic parameter, EPDM was considered as an elastomer model. With the elastic modulus of EPDM and contact pressure of the tribosystem it is possible to determine the load and radius of the indenter in a representative laboratory experiment. The surface tension between EPDM and steel was calculated from contact angle measurements. It was concluded that despite the attractive forces between the surfaces, the effect on the contact area is low, and therefore adhesion will not be considered in calculating the real contact area.

The contact models available in literature are valid for isotropic viscoelastic contact or for elastic anisotropic contact, but they do not consider both behaviours simultaneously. Therefore under the previously mentioned assumptions, a viscoelastic orthotropic contact model has to be developed.

To characterize the friction behaviour, the conditions that represent the present tribo-system were determined. The sliding velocity, the applied load, the various values of radius of the indenter and the environmental temperature allowed the tribological behaviour to be reproduced in a pinon-disk experiment. With the sliding friction experiments it was possible, by adding a thin layer of oil, to isolate the deformation (ploughing) mechanism of friction. The deformation mechanism of friction was found to be very low in comparison with the adhesion mechanism and will therefore be neglected in the present study. A friction model based on the adhesion mechanism will be developed, so an understanding of the behaviour of the contact area and of the shear stresses in the surface or interface of the surface is required. 
Chapter 3

In summary, considerations regarding the contact and friction behaviour were made in the present chapter. These considerations allow the tribological behaviour to be reproduced in an experimental setup, determining the most influencing mechanism of friction. To model the frictional behaviour the contact area and shear stress need to be known. Chapter 4 focuses on the contact model and Chapter 5 focuses on the shear stresses in the contact between the surfaces. 


\section{Chapter 4 Contact model}

Fibre reinforced elastomers behave like viscoelastic materials due to the polymer matrix and as anisotropic materials due the preferred orientation of the fibres. To determine the contact between a rigid spherical indenter and an anisotropic viscoelastic material, a contact model that considers the effect of both behaviours is needed. Two contact models that describe anisotropic material behaviour were extended to include viscoelastic behaviour. The difference of applicability between the two contact models is the degree of anisotropy. The first contact model is most suitable for reinforced materials with low degree of effective anisotropy and the second contact model is more suitable at high degrees of anisotropy. A low degree of anisotropy means that the material properties, such as elastic modulus, shear modulus and Poisson's ratio, have nearly the same values in each principal direction. A high degree of anisotropy means that the elastic modulus, shear modulus and Poisson's ratio in the three principal directions can be very different.

\section{Influence of surface properties}

The contact behaviour in this work depends on the material properties of the elastomer composite. Sliding friction can produce a modification of the mechanical properties of the surface. This was studied by Deladi [46], who showed an interfacial layer created on polyurethane during friction experiments. In the tribo-system studied, a modification of the material properties in the area near the surface was observed. Details of this layer and its behaviour can be seen in Chapter 5 . The material properties of the surface influence the contact behaviour when the contact area and the thickness of the layer are in the same order of magnitude. In the present study, the radius of the contact area, order $\mathrm{mm}$, is much larger than the thickness of the modified surface layer, order $\mu \mathrm{m}$, therefore the effect of the layer properties in the calculation of the contact area can be neglected [52].

Therefore a contact model that considers the anisotropic and viscoelastic behaviour, neglecting the surface layer, will be developed. In the present chapter, the basic procedure for the two anisotropic elastic contact models will be presented, followed by a short discussion and the extension to include viscoelastic behaviour. 
Chapter 4

\section{Material Model}

To compare the results obtained using the developed contact model with models available in literature -elastic anisotropic and isotropic viscoelasticthe elastic and viscoelastic properties of EPDM will be considered as reference. The elastic material properties were measured for EPDM on a tensile tester and the viscoelastic properties were measured using Dynamic Mechanical Analysis (DMA). The elastic modulus of the EPDM sample is $\mathrm{E}=4.39 \pm 0.14 \mathrm{MPa}$ and the mean creep compliance of the material is expressed as a series of discrete exponential terms [47] given by:

$$
\phi(t)=\phi_{r}-\sum_{i=1}^{3} \phi_{i} \exp \left(\frac{-t}{\lambda_{i}}\right)
$$

where $\phi_{r}$ indicates the creep compliance at a fully relaxed state and $\lambda_{i}$ indicates the retardation times. For the EPDM used in this study, these parameters are summarized in Table 4.1.

Table 4.1: Compliance coefficients and Retardation times for EPDM.

\begin{tabular}{ccc}
\hline $\mathrm{i}$ & $\phi_{i}(\mathrm{~Pa}-1)$ & $\lambda_{i}(\mathrm{~s})$ \\
\hline$\phi_{r}$ & $3.493 \cdot 10^{-7}$ & \\
1 & $4.692 \cdot 10^{-8}$ & 0.0064 \\
2 & $4.903 \cdot 10^{-8}$ & 0.0713 \\
3 & $8.232 \cdot 10^{-8}$ & 0.7284 \\
\hline
\end{tabular}

To facilitate the discussion regarding the anisotropy of the material, a system of coordinates is defined. The direction of indentation corresponds to the $z$-axis and the plane in which the contact occurs, where the contact area forms, corresponds to the $x-y$ plane, as seen in Fig. 4.1. 


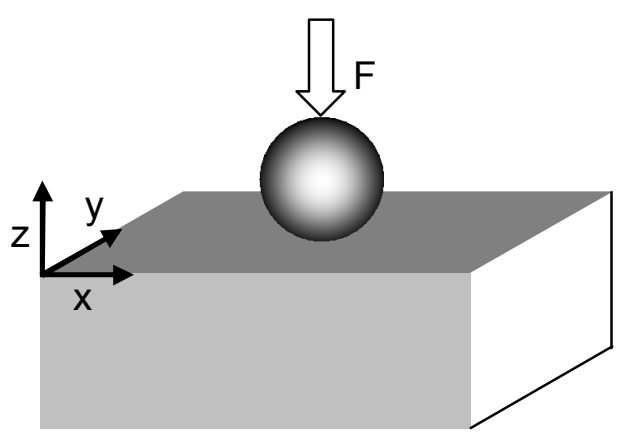

Fig. 4.1: Schematic of the coordinates system used in the present chapter.

The developed viscoelastic-anisotropic contact model considers a static contact. In this thesis, the sliding contact of a fibre reinforced elastomer is studied. Therefore a study of the size and shape of contact area due to the sliding speed was performed from which a correction factor to consider the sliding velocity dependency was found.

\subsection{The static contact area}

Sun et al. [17, 18], proposed an approximation for the deformation of an anisotropic elastic material that is indented by a rigid sphere. The contact area is given by a circle in which the radius is defined by the Hertz solution, with the equivalent elastic modulus defined as the equivalent elastic modulus of the anisotropic material in the direction of indentation, see Eq. 4.2 .

$$
a=\left(\frac{3 R F_{N}}{4 E_{*_{z}}}\right)^{1 / 3}
$$

Willis [19] and Swanson [20] developed a model describing anisotropic elastic contact for a point load, and extended this to consider a pressure distribution. The details of this procedure can be found in Appendix $G$. The contact area is given by an ellipse of principal axes $a_{x}$ and $a_{y}$, defined as:

$$
a_{x}=\left(\frac{3 R F_{N}}{4}\right)^{1 / 3} I_{1}^{1 / 3} \text { and } a_{y}=\left(\frac{3 R F_{N}}{4}\right)^{1 / 3} \frac{I_{2}^{1 / 2}}{I_{1}^{1 / 6}}
$$

where $I_{1}$ and $I_{2}$ are the numerical solutions of two contour integrals, dependent on nine material properties of the orthotropic material and a parameter $\varepsilon=a_{x} / a_{y}$. The integrals are defined in Eq. 4.4 and Eq. 4.5, 
where $\tilde{u}_{z}$ is the Fourier transform of the normal displacement due to a point load and depends on all the material properties. Details are given in Appendix G.

$$
\begin{aligned}
& I_{1}=\int_{0}^{2 \pi} \tilde{u}_{z}(\varepsilon \cos \theta, \sin \theta)(\cos \theta)^{2} d \theta \\
& I_{2}=\int_{0}^{2 \pi} \tilde{u}_{z}(\varepsilon \cos \theta, \sin \theta)(\sin \theta)^{2} d \theta
\end{aligned}
$$

It can be seen that the form of the solution of the radius of the contact area is similar to the solution by Hertz, and the only difference is in the term corresponding to the elastic material properties. In the contact model by Sun and co-workers [18] the elastic properties of the anisotropic material are defined by the properties in only one direction, while in the contact model by Willis [19] and Swanson [20] the material properties of the anisotropic material are defined by the material properties in each principal direction. In the case of an orthotropic material there are nine: three elastic moduli, three shear moduli and three Poisson's ratios.

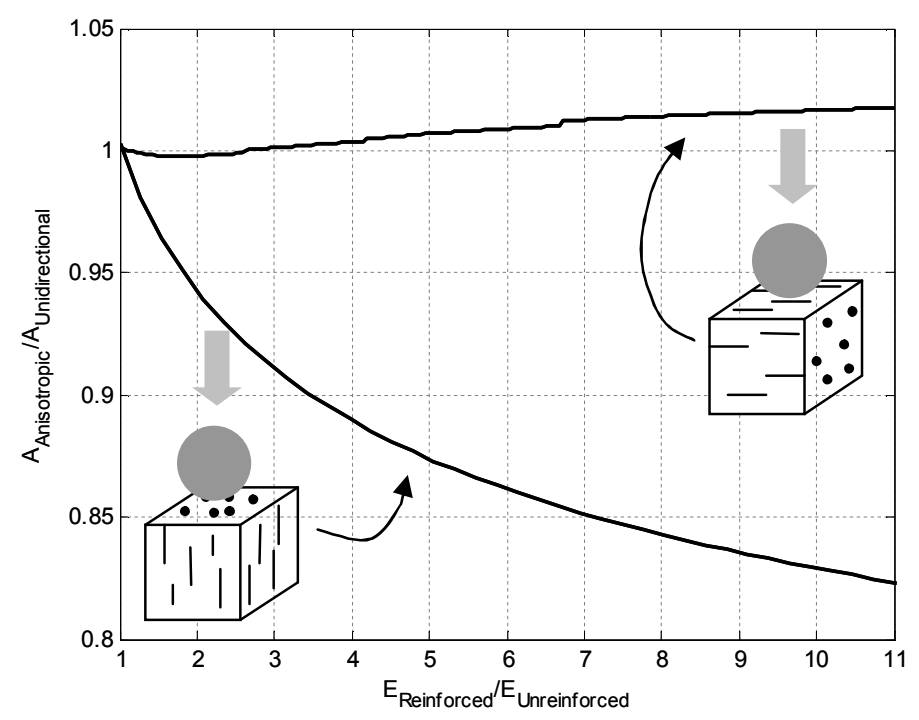

Fig. 4.2: Ratio of the contact areas as calculated using both models, for different amounts of reinforcement. 
The two contact models are compared by calculating the ratio of the contact areas using each elastic contact model, see Fig. 4.2, $A_{\text {unidirectional }}$ over $A_{\text {anisotropic }}$ for a transversally isotropic material with an increased elastic modulus in the reinforced direction. The area $A_{\text {unidirectional }}$ is calculated using the contact model of Sun et al. [17, 18], whilst the area $A_{\text {anisotropic }}$ is calculated according to Willis [19], in which the shear modulus in the nonisotropic directions is obtained from the elastic modulus in the direction of indentation according to:

$$
G=\frac{E_{z}}{2(1+v)}
$$

The upper curve in Fig. 4.2 shows that for an increased elastic modulus (i.e. reinforcement) in the plane of indentation, the difference between the two contact models is less than $3 \%$. For a material with reinforcement in the direction of indentation the differences are larger and depend greatly on the amount of reinforcement. A comparison between the two contact models was made by Swanson [20], who concludes that the accuracy of the contact model by Sun and co-workers is good for low degrees of anisotropy of the material. The results in Fig. 4.2 are in agreement with the findings of Swanson, however, it is important to note that the applied value for the shear modulus of the non-isotropic planes has a large influence on the results. When it is defined differently, it is possible to obtain larger differences between the two contact models.

\subsubsection{Extension to viscoelasticity of Sun's contact model}

The advantage of the contact model by Sun and co-workers is the low number of needed parameters in comparison with the more accurate contact model by Willis and Swanson. Chen [53] showed that under pure normal loading, the shape of the normal displacement and the shape of the pressure distribution are the same for anisotropic and isotropic materials, so only the absolute value can differ. Therefore the assumption made by Sun et al. $[17,18]$ to model the anisotropic behaviour in a similar way to the isotropic behaviour in the surface of contact appears to be valid. Furthermore, Chen [53] shows that the stress distribution inside the anisotropic elastic body is symmetrical in the case of an orthotropic material in which one of its principal axes coincides with the direction of indentation. Hence, the anisotropic approximation by Sun et al. $[17,18]$ is well suited to approximating the occuring stress distribution for anisotropic materials with an isotropic indentation plane. 
A common approach in linear viscoelastic theory (see for example Lee \& Radok [11], Ting [14]) is to express the viscoelastic solution of the isotropic contact problem in terms of the elastic solution. Following this thought, the stress distribution inside an orthotropic visco-elastic material can be described based on the orthotropic elastic solution. This means that, for an anisotropic viscoelastic material with a low ratio of reinforcement (i.e. $E_{z} / E_{x}$ is nearly 1), the time dependent contact area can be calculated by combining the solution of the viscoelastic contact problem with the elastic model of Sun et al. [17, 18].

A viscoelastic anisotropic material is characterized by a stress relaxation function $\psi_{z}$ and a creep compliance function $\phi_{z}$, both measured in the direction of indentation. For the loading phase of the contact, i.e. an increasing contact area, the pressure distribution in the contact area, due to indentation with a rigid sphere with a radius of $R$, is given by Eq. 2.7. The total force is given by:

$$
F_{N}(t)=\frac{8}{3 R} \int_{0^{-}}^{\infty} \psi_{z}\left(t-t_{*}\right) \frac{\partial}{\partial t_{*}}\left(a^{3}\left(t_{*}\right)\right) d t_{*}
$$

Assuming that the force is defined by a Heaviside function as $F_{N}(t)=H(t) F_{N}$, Eq. 4.7 can be inverted and the contact area can be calculated, because the creep compliance $\phi_{z}$ and stress relaxation function $\psi_{z}$ are related according to:

$$
\psi_{z}(s) \phi_{z}(s)=\frac{1}{s^{2}}
$$

The radius of the contact area is:

$$
a(t)=\left(\frac{3 R F_{N}}{8} \phi_{z}(t)\right)^{1 / 3}
$$

Hence, the contact area between a smooth rigid indenter and a deforming viscoelastic anisotropic body is a circle with time dependent dimensions and is characterized by the viscoelastic properties of the anisotropic material in the indentation direction only.

\subsubsection{Elastic versus viscoelastic contact model}

The results from the viscoleastic contact model are compared with those obtained with the purely elastic case for a rigid ball with a radius 
$R=12.5 \mathrm{~mm}$ against a deforming flat surface at a normal load of $2 \mathrm{~N}$. Fig. 4.3 shows the calculated contact radius for EPDM, calculated for the purely elastic case and when taking into account the viscoelastic behaviour. The difference between the two models occurs immediately after loading and decreases with increasing time; in this case, after 1 second the difference between the viscoelastic and elastic contact area is less than $2 \%$. For contacts that occur at short time scales with respect to the relaxation time of the material, a viscoelastic contact model gives a more accurate description of the contact area. For long time scales, the contact areas calculated using a viscoelastic contact model or an elastic contact model are similar. Note that, as the EPDM material is isotropic, these results are equal to those obtained using isotropic contact models.

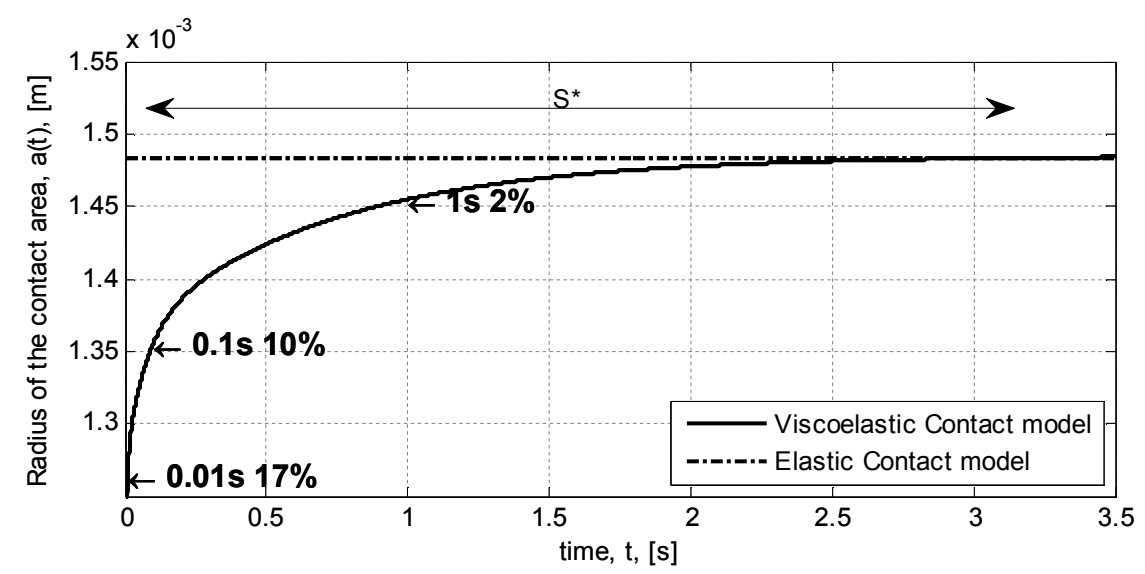

Fig. 4.3: Radius of the contact area, a(t), calculated using the elastic contact model of Hertz (dashed line), and the viscoelastic contact model (solid line). After a time $\mathrm{S}^{*}$, the viscoelastic contact area equals the elastic contact area.

\subsubsection{Influence of different parameters of the creep compliance function}

Different parameters of the creep compliance function have been modified to study their influence on the final creep compliance and on the contact behaviour.

\section{Creep compliance function}

To study the influence of the creep compliance function on the radius of the contact area, the creep parameters involved in the compliance function, given in Table 4.1, have been multiplied by a factor of 2, 3, 4, 5 and 6 respectively. The results are shown in Fig. 4.4. The solid black line shows 
the radius of the contact as a function of time, calculated with the creep compliance corresponding to the material model. The dashed black line shows the contact area, calculated assuming elasticity. The various dashed grey lines show the radius of the contact area calculated at various factors of the creep compliance. As the creep compliance increases, the radius of the contact area also increases, which can be translated to a loss of stiffness of the material and therefore a higher indentation under the same loading conditions.

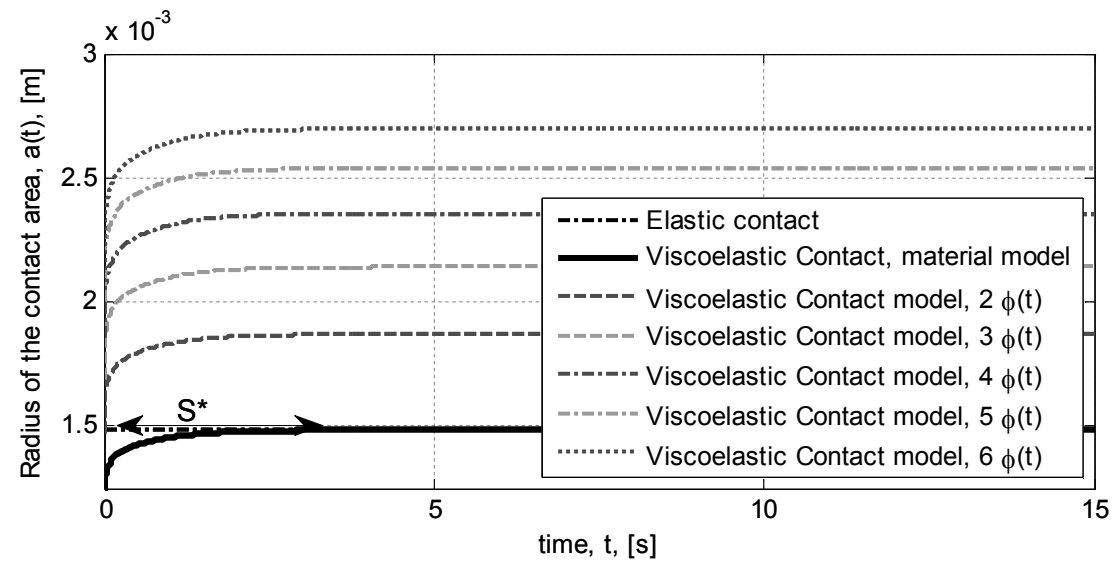

Fig. 4.4: Influence of the creep compliance function on the radius of the contact area.

\section{Fully relaxed creep compliance}

The influence of the creep compliance at a fully relaxed state, defined by $\phi_{r}$ in Table 4.1, can be analyzed by multiplying the relaxed creep compliance, $\phi_{r}$, by a factor of $2,3,4,5$ and 6 . At long time scales, the values obtained for the contact radius in each case will be equal to those shown in Fig. 4.4. At short time scales the results differ.

Fig. 4.5 shows the time it takes for the contact radius $a(t)$ to reach the stable state (time $S^{*}$, see Fig. 4.4), i.e. where the radius of the contact area calculated by the viscoelastic contact model has the same value as the radius of the contact area calculated by the elastic contact model, $a_{\text {viscoelastic }}=a_{\text {elastic }}$. It can be seen that a higher multiplying factor of the relaxed creep compliance reduces the time $S^{*}$.

Fig. 4.6 shows ratios of the radius of the contact area at different instants of time $(0.1 \mathrm{~s}, 0.7 \mathrm{~s}, 1.5 \mathrm{~s})$ with the radius of the contact area at equilibrium, for the different multiplying factors used for $\phi_{r}$. The multiplying factor 1 
indicates the original creep function, factor 2 indicates $2 \phi_{r}$ and so on. These figures clearly show that an increase of just the relaxed creep compliance, whilst keeping the other creep parameters constant, does affect the rate of increase of the contact area but not the final size of the contact in the relaxed state.

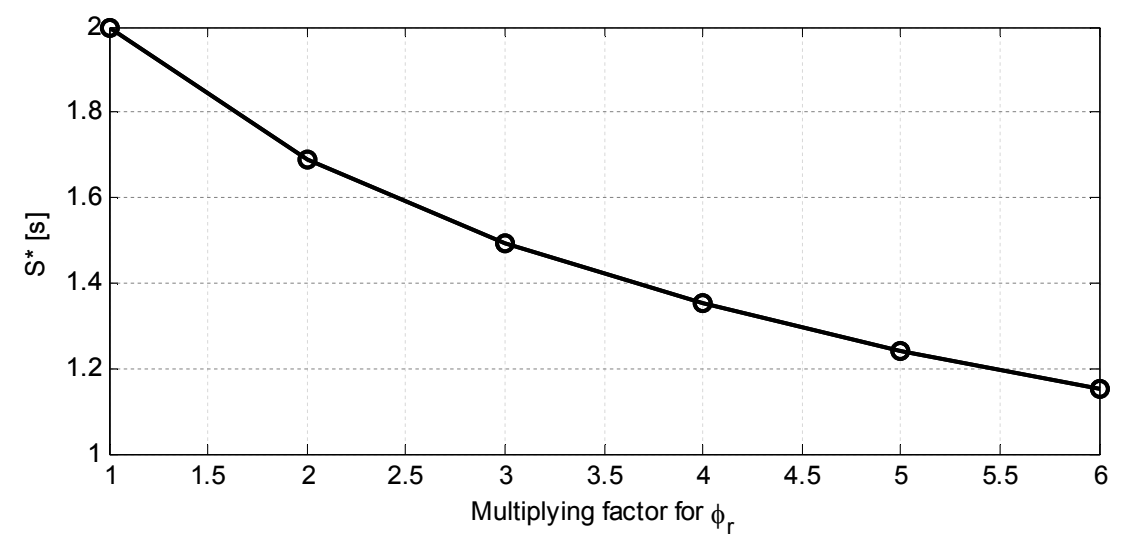

Fig. 4.5: Influence of the relaxed creep compliance $\phi_{r}$, on the time $S^{*}$ where

$a_{\text {viscoelastic }}=a_{\text {elastic }}$.

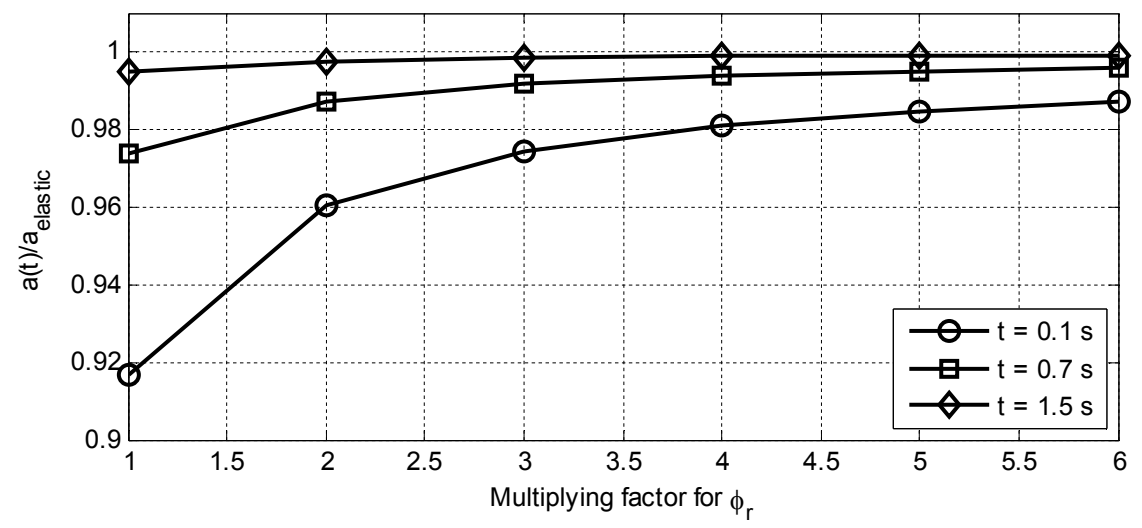

Fig. 4.6: Influence of the relaxed creep compliance $\phi_{r}$, on the radius of the contact area.

\section{Retardation times}

Fig. 4.7 shows the influence of the retardation times on the creep compliance function; the retardation times, $\lambda_{i}$ in Table 4.1, were multiplied 
by 10,100 and 1000 respectively. Fig. 4.8 shows the resulting effects on the radius of the contact patch. It can be seen that an increase on all the retardation times produces a decrease of the speed at which the creep compliance reaches the stable state, corresponding to the time $S^{*}$.

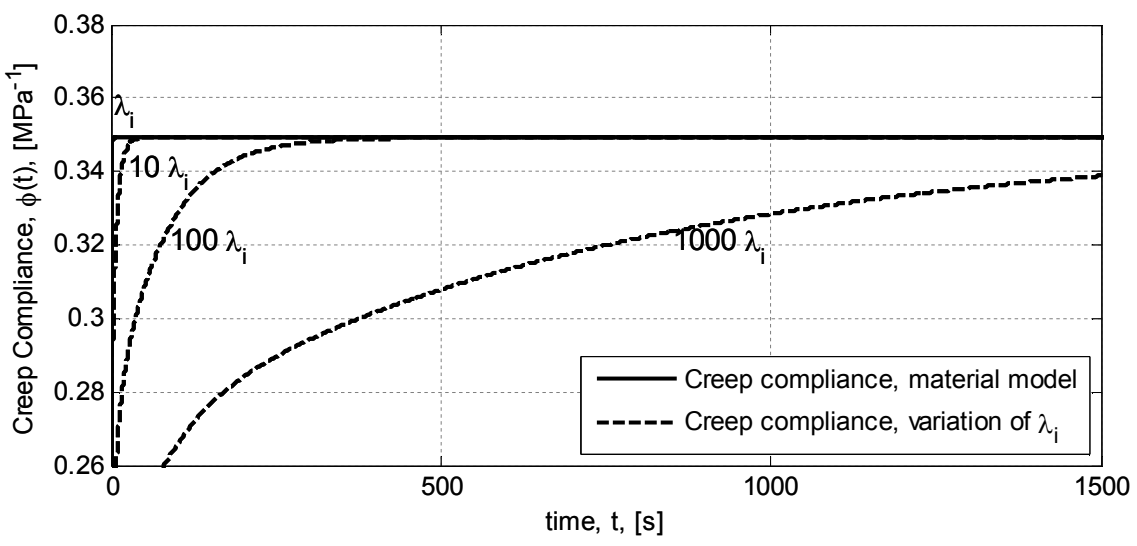

Fig. 4.7: Influence of retardation times in the creep compliance function. Where 10 $\lambda_{\mathrm{i}}$ means $10 \lambda_{1}, 10 \lambda_{2}$ and $10 \lambda_{3}$.

Fig. 4.8 shows that an increase in the retardation times slows down the increase of the contact area in time. Comparing the contact between a rigid sphere and a 'fast' viscoelastic material with that of a rigid sphere and a 'slow' viscoleastic material, i.e. the viscoelastic materials differ only in the retardation times, will result in the same contact area at the long time scale, but the sphere indents more slowly into viscoelastic materials that have higher retardation times. 


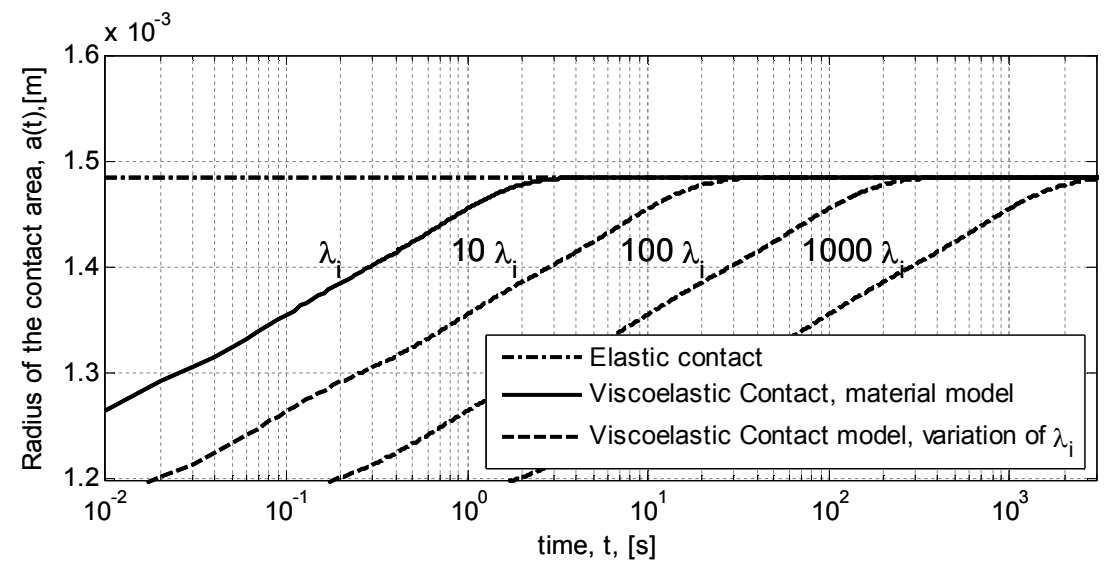

Fig. 4.8: Radius of the contact area, at different factors of the retardation times; 10 $\lambda_{i}$ means $10 \lambda_{1}, 10 \lambda_{2}$ and $10 \lambda_{3}$.

The influence of the separate retardation times $\lambda_{1}, \lambda_{2}$ and $\lambda_{3}$ is studied by independently multiplying each retardation time by a factor of 100 . As the creep compliance coefficients are of the same order, it is possible to compare the influence of each retardation time on the contact area.

The results on the creep compliance function can be seen in Fig. 4.9, where the black line shows the creep compliance function of the material model and the grey line show the creep compliance resulting from modifications of the retardation times. The effects of the variation in creep compliance function on the radius of the contact area are shown in Fig. 4.10. The black line indicates the contact radius calculated with the creep compliance of the material model, the grey lines show the radius of the contact area calculated with the creep compliance at different varitions of the retardation time. As seen before, the modifications of the retardation times affect neither the final value of the creep compliance function nor the final size of the contact area. However, at short time scales the results differ significantly.

At very short time scales $\left(10^{-1} \mathrm{~s}\right)$ the contact area with the smaller retardation time modified $\left(\lambda_{1}\right)$ approaches the contact area of the material model, but at long timescales $\left(10^{1} \mathrm{~s}\right)$ a modification of the higher retardation time, causes higher differences (contact area increases slowly with time). 


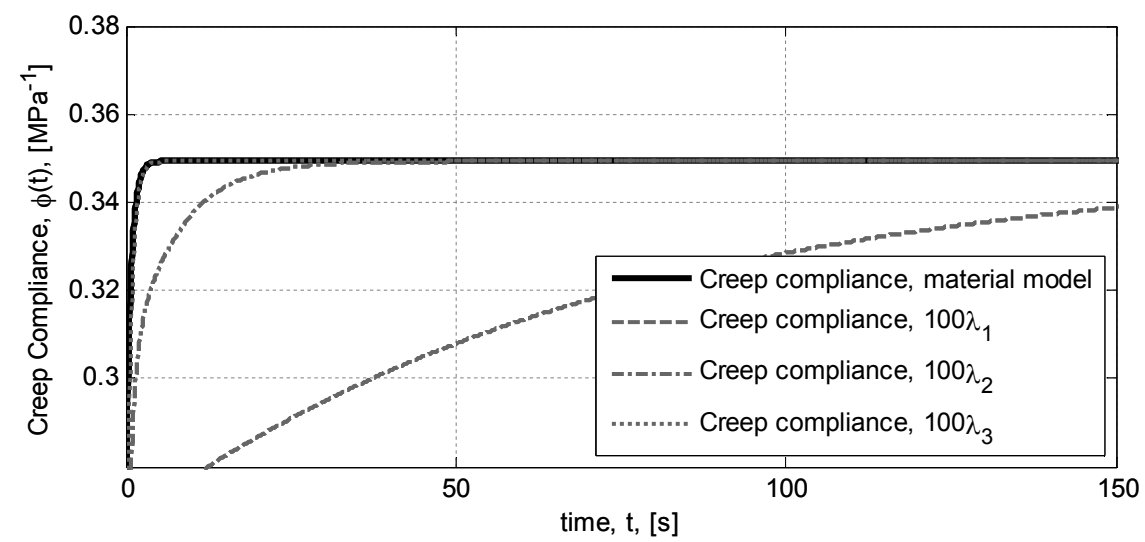

Fig. 4.9: Influence of individual retardation times on the creep compliance function.

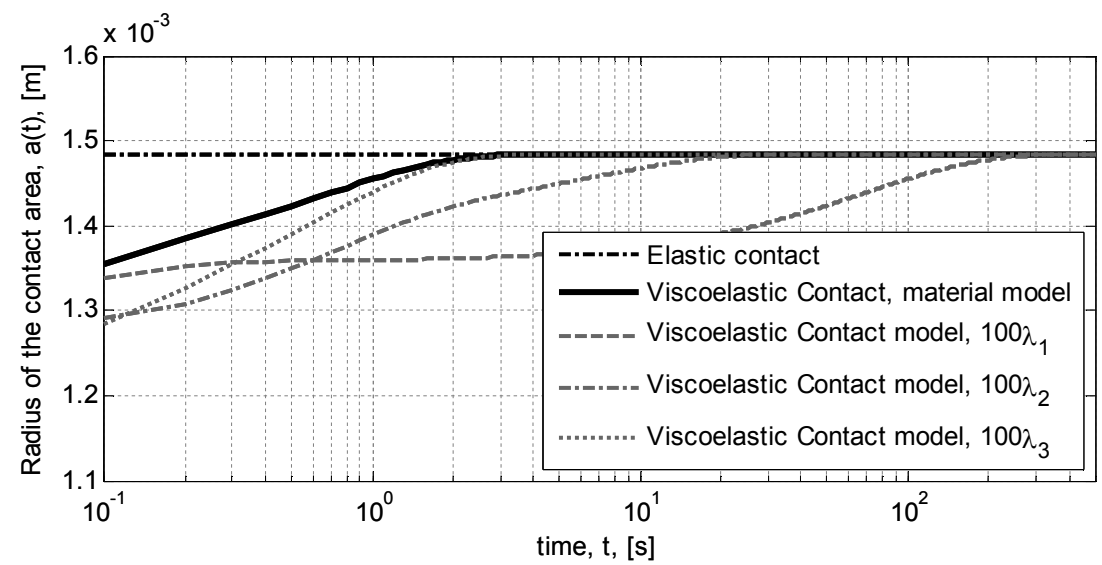

Fig. 4.10: Radius of the contact area for different retardation times.

\subsubsection{Conclusions}

A model that describes the contact behaviour of viscoelastic weakly anisotropic materials has been developed. The contact model is based on the fact that, under weak anisotropy, the contact behaviour can be described by taking the properties of the material in the normal or loading direction. Similar to common practice in viscoelasticity, where the solution to the contact problem is expressed in terms of the corresponding elastic contact problem, the solution in orthotropic viscoelasticity can be based on the orthotropic elastic case. A comparison between the contact areas calculated using an elastic contact model and a viscoelastic contact model was made. The results coincide for long time scales. From the parameter 
study on the creep behavior it can be concluded that an increase in the creep compliance function results in an increase of the size of the contact area and that an increase in the retardation times of the model results in a slower increase in the size of the contact area.

The results show that at short time scales the real area of contact as calculated using the viscoelastic material model can be significantly smaller than when assuming fully elastic behaviour. Such fast contacts occur in for instance rolling tyre-road contacts, where the contact time between rubber and stone is rather small, much less than one second.

\subsubsection{Extension to viscoelasticity of the Willis model}

Similarly to the procedure of Willis [19] and Swanson [20], to obtain a solution to the orthotropic viscoelastic contact problem, first the auxiliary problem of a concentrated normal load applied to a viscoelastic orthotropic half-space is solved. The solution involves separation of variables in time and space, the temporal solution is found and the spatial solution used corresponds to the solution of Willis [19] and Swanson [20]. To calculate the contact problem of a viscoelastic anisotropic material indented by a rigid spherical indenter, the contact area and pressure distribution used are the ones defined by Willis [19] and Swanson [20].

\section{Solution of a point load in an anisotropic viscoelastic material}

The equations to solve are the following [19, 20, 14]:

- The stress and displacement must satisfy the equation of equilibrium:

$$
\frac{\partial \sigma_{i j}\left(x_{i}, t\right)}{\partial x_{j}}=0
$$

- The strain-displacement relation:

$$
\varepsilon_{i j}=\frac{1}{2}\left(\frac{\partial u_{i}}{\partial x_{j}}+\frac{\partial u_{j}}{\partial x_{i}}\right)
$$

- The linear viscoelastic stress-strain relation, valid for anisotropic materials:

$$
\sigma_{i j}\left(x_{i}, t\right)=\int_{0}^{t} \psi_{i j k l}\left(t-t_{*}\right) \frac{\partial \varepsilon_{k l}\left(x_{i}, t_{*}\right)}{\partial t_{*}} d t_{*}
$$


Where $\psi_{i j k l}(t)$ is a fourth order time dependent tensor that corresponds to the stress relaxation functions of the material. The indices indicate the $x, y$ and $z$ directions, and $x_{i}$ denotes the spatial variable thus $x_{x}=x, x_{y}=y$ and $x_{z}=z$.

It is considered that there is full contact within the contact area between the half-space and the indenter, i.e. no separation occurs, and the problem is quasi-static. Assuming separation of variables between space and time enables the definition of the displacement field $\boldsymbol{u}$, the strain $\varepsilon$ and the stress $\boldsymbol{\sigma}$ as respectively:

$$
\begin{aligned}
u_{i}\left(x_{i}, t\right) & =u_{i}\left(x_{i}\right) \bar{u}(t) \\
\varepsilon_{i j}\left(x_{i}, t\right) & =\varepsilon_{i j}\left(x_{i}\right) \bar{u}(t) \\
\sigma_{i j}\left(x_{i}, t\right) & =\sigma_{i j}\left(x_{i}\right) \bar{F}(t)
\end{aligned}
$$

In the separation of variables defined in Eq. 4.13 to Eq. $4.15, F_{N}$ is a constant and indicates the applied normal load, $\bar{u}(t)$ and $\bar{F}(t)$ indicates the time functions of the displacement field and applied load respectively, they are dimensionless and the dimension is kept by the spatial variables.

The dimensionless applied normal load, $\bar{F}(t)$, is defined by the Heaviside step function as:

$$
\bar{F}(t)=H(t)= \begin{cases}0 & t<0 \\ 1 & t \geq 0\end{cases}
$$

For $t<0$, the viscoelastic half-space is stress-free and at $t \geq 0$ the spherical indenter is pressed into the half-space.

Using the assumption of separation of variables, it is possible to rewrite $\psi_{i j k l}(t)$ as:

$$
\psi_{i j k l}(t)=\bar{\psi}(t) \cdot \psi_{i j k l}
$$

where the constant tensor $\psi_{i j k l}$ will behave similar to the stiffness tensor, keeping the dimensions and $\bar{\psi}(t)$ gives information regarding the variation of material properties as a function of time, working as a normalized stress relaxation function. With this it is possible to use $\psi_{i j k l}$ as the common 
stiffness tensor found in elasticity $C_{i j k l}$, therefore the stress strain relationship in the spatial variables can be defined as $\sigma_{i j}\left(x_{i}\right)=\psi_{i j k l} \varepsilon_{k l}$.

To solve the time dependent component of the displacement field, $\bar{u}(t)$, one of the stress boundary conditions is used, which is defined in the spatial and time variables and where $\delta$ refers to the Dirac delta function with reciprocal dimensions to the dimension of $x$ or $y$. The boundary condition used is given by:

$$
\sigma_{z z}(x, y, z=0, t)=-\delta(x) \delta(y) F_{N} \bar{F}(t)
$$

The time dependent boundary condition is then:

$$
\int_{0}^{t} \bar{\psi}\left(t-t_{*}\right) \frac{\partial \bar{u}\left(t_{*}\right)}{\partial t_{*}} d t_{*}=\bar{F}(t)
$$

The normalized creep compliance function $\bar{\phi}(t)$ is related to the normalized stress relaxation function $\bar{\psi}(t)$ by:

$$
\int_{0}^{t} \bar{\psi}\left(t-t_{*}\right) \bar{\phi}\left(t_{*}\right) d t_{*}=t
$$

Inverting the time dependent boundary condition of Eq. 4.19 and with the relation between stress relaxation and creep compliance given in Eq. 4.20, the time dependency of the displacement field $\bar{u}(t)$ can be described by:

$$
\bar{u}(t)=\int_{0}^{t} \bar{\phi}\left(t-t_{*}\right) \frac{\partial}{\partial t_{*}} H\left(t_{*}\right) d t_{*}=\bar{\phi}(t)
$$

The solution of the spatial displacement in the Fourier domain is obtained identically to Willis [19], and Swanson [20], see Eq (G.9), Appendix G.

\section{Solution of the contact problem}

The time dependent contact pressure is given by Eq. 4.22, where the contact area is given by an ellipse of principal axes $a_{x}(t)$ and $a_{y}(t)$. As the spatial and temporal variables are independent, the principal axes of the contact area depend on time in the same way and can be written as $a_{x}(t)=a_{x} \bar{a}(t)$ and $a_{y}(t)=a_{y} \bar{a}(t)$. The time dependency of the pressure 
distribution is given by the dimensionless parameter $\bar{p}(t)$ and the peak contact pressure is given by $p_{0}$.

$$
p(x, y, t)=\bar{p}(t) p_{0}\left(1-\left(\frac{x^{2}}{a_{x} \bar{a}(t)}+\frac{y^{2}}{a_{y} \bar{a}(t)}\right)\right)^{1 / 2}
$$

The superposition of the spatial and temporal solution for the normal displacement is given by Eq. 4.23 , where $\bar{u}\left(t-t_{*}\right)$ is the dimensionless time dependent displacement field at time $t$, due to a contact pressure that occurred at an instant $t_{*}$, and $\eta_{1}, \eta_{2}$ are $\cos \theta$ and $\sin (\theta)$ respectively.

$u_{z}(x, y, 0, t)=$

$\int_{0}^{t} \frac{\bar{p}\left(t_{*}\right)}{\bar{a}\left(t_{*}\right)} \frac{\partial \bar{u}\left(t-t_{*}\right)}{\partial t_{*}} d t_{*} \cdot \frac{\pi p_{0} a_{y}}{4} \int_{0}^{2 \pi} \tilde{u}_{z}\left(\varepsilon \eta_{1}, \eta_{2}\right)\left[1-\left(\frac{\eta_{1} x}{a_{x}}+\frac{\eta_{2} y}{a_{y}}\right)^{2}\right] d \theta$

On the right of the product is the spatial solution for the orthotropic contact problem given in more detail in Appendix G.

The relative displacement of the indenter into the half space is given by $\delta(t)$ in:

$$
u_{z \text {-indenter }}+u_{z \text {-half-space }}=\delta(t)-\left(A x^{2}+B y^{2}+2 C x y\right) F_{N} \bar{F}(t)
$$

For the special case of a rigid spherical indenter, the terms $A, B$ and $C$ in Eq. 4.24 are both defined by Eq. 4.25 [10].

$$
A=B=\frac{1}{2 R} \quad \text { and } \quad C=0
$$

Recognizing terms between Eq. 4.23 and Eq. 4.24, is obtained:

$$
\frac{F_{N} \bar{F}(t)}{2 R}=\int_{0}^{t} \frac{\bar{p}\left(t_{*}\right)}{\bar{a}\left(t_{*}\right)} \frac{\partial \bar{u}\left(t-t_{*}\right)}{\partial t_{*}} d t_{*} \cdot \frac{\pi p_{0} a_{\mathrm{y}}}{4 a_{x}^{2}} \int_{0}^{2 \pi} \widetilde{u}_{z}(\theta) \cos ^{2}(\theta) d \theta
$$

An auxiliary function is defined, this is given in Eq. 4.27:

$$
\mathrm{f}(t)=\frac{\bar{p}(t)}{\bar{a}(t)}
$$


With Eq. 4.27 and using the commutative properties of convolution it is possible to invert the time dependent terms of Eq. 4.26, obtaining a solution for the time dependency of the contact area as:

$$
\int_{0}^{t} \mathrm{f}^{-1}\left(t-t_{*}\right) \frac{\partial H\left(t_{*}\right)}{\partial t_{*}} d t_{*}=\mathrm{f}^{-1}(t)=\bar{u}(t)=\bar{\phi}(t)
$$

Where $f^{-1}(t)$ indicates the inverse function of $f(t)$. The time dependent part of the peak contact pressure can be replaced by the time dependent part of the total contact force, as:

$$
\bar{F}(t)=\bar{p}(t) \bar{a}^{2}(t)
$$

The time dependency of the contact area is given by:

$$
\bar{a}(t)=[\bar{F}(t) \cdot \bar{\phi}(t)]^{1 / 3}
$$

As the total pressure is given by $F_{N}(t)=F_{N} \bar{F}(t)$, where $\bar{F}(t)$ follows a Heaviside function, the solution of the viscoelastic orthotropic contact can be expressed as the multiplication of the contact solutions in time and space [20]. The contact area is given by an elliptical contact area of axes $a_{x}(t)$ and $a_{y}(t)$ given by:

$$
\begin{gathered}
a_{x}(t)=\left(\frac{3 R F}{4}\right)^{1 / 3}\left[I_{1} \cdot \bar{\phi}(t)\right]^{1 / 3}, \\
a_{y}(t)=\left(\frac{3 R F}{4}\right)^{1 / 3}\left(\frac{I_{2}^{1 / 2}}{I_{1}^{1 / 6}}\right)[\bar{\phi}(t)]^{1 / 3},
\end{gathered}
$$

The normalized creep compliance function, $\bar{\phi}(t)$, has been normalized with respect to the fully relaxed compliance, $\phi_{r}$. Therefore in the elastic case, the normalized creep compliance function will have a constant, $\bar{\phi}(t)=1$, and from the definition of creep compliance in [14], the contact area in the viscoelastic isotropic case is given by:

$$
a(t)=\left(\frac{3 R F}{4}\right)^{1 / 3}\left[\frac{1-v^{2}}{E} \cdot \bar{\phi}(t)\right]^{1 / 3} \text { or } a(t)=\left(\frac{3 R F}{4}\right)^{1 / 3}\left[\frac{\phi(t)}{2}\right]^{1 / 3}
$$




\subsubsection{Influence of the normalized creep function}

In Eq. 4.33 the elastic behaviour is represented by $E$ and the viscous by $\bar{\phi}(t)$. To study the influence of the viscous or time dependent behaviour, the normalized creep functions of several materials (EPDM, PU [46], PMMA [54] and PC [54]) are presented in Fig. 4.11. These normalized creep functions will be multiplied with the elastic properties of EPDM to calculate the contact area; the results are shown in Fig. 4.12. In Fig. 4.11 it is shown that at long times scales, the creep compliance function approaches unity, the speed of this approach depends mainly on the retardation times of the creep function as seen in the parametric study shown in Fig. 4.4 to Fig. 4.10. The creep function of a purely elastic material is also shown, which is described by a constant value, one.

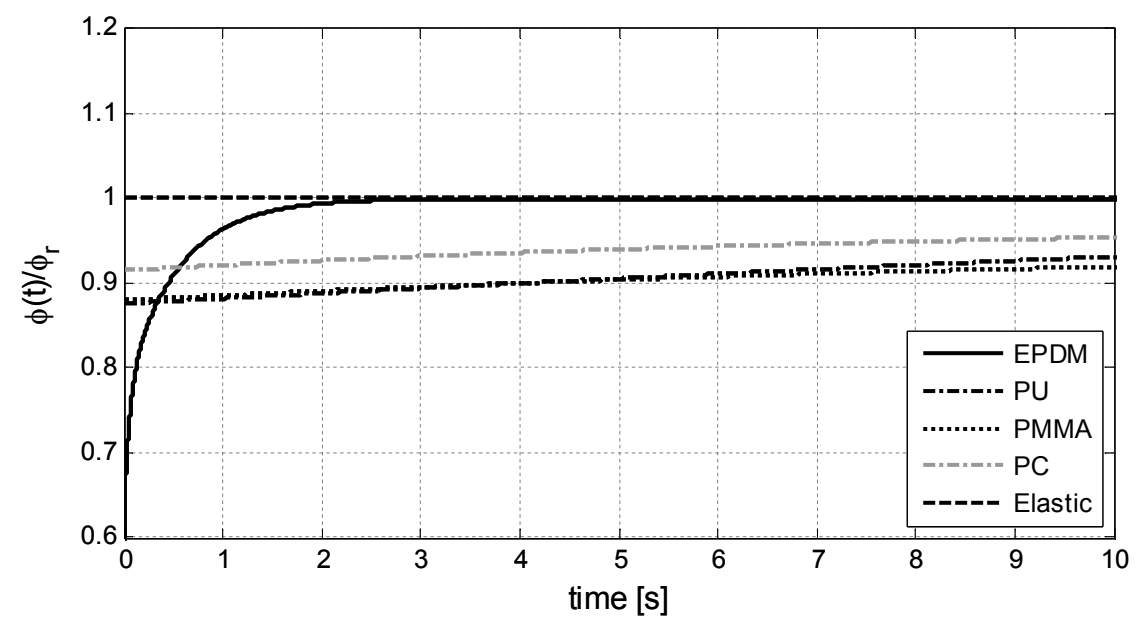

Fig. 4.11: Normalized creep function, for different materials.

The differences in the calculation of the contact area, due to different normalized creep functions, correspond to the different retardation times that each material has. The time dependency of the contact area is influenced by the normalized creep compliance, but the magnitude of the contact area is influenced by the elastic material parameters used in the elastic response of the contact area $[19,20]$. 


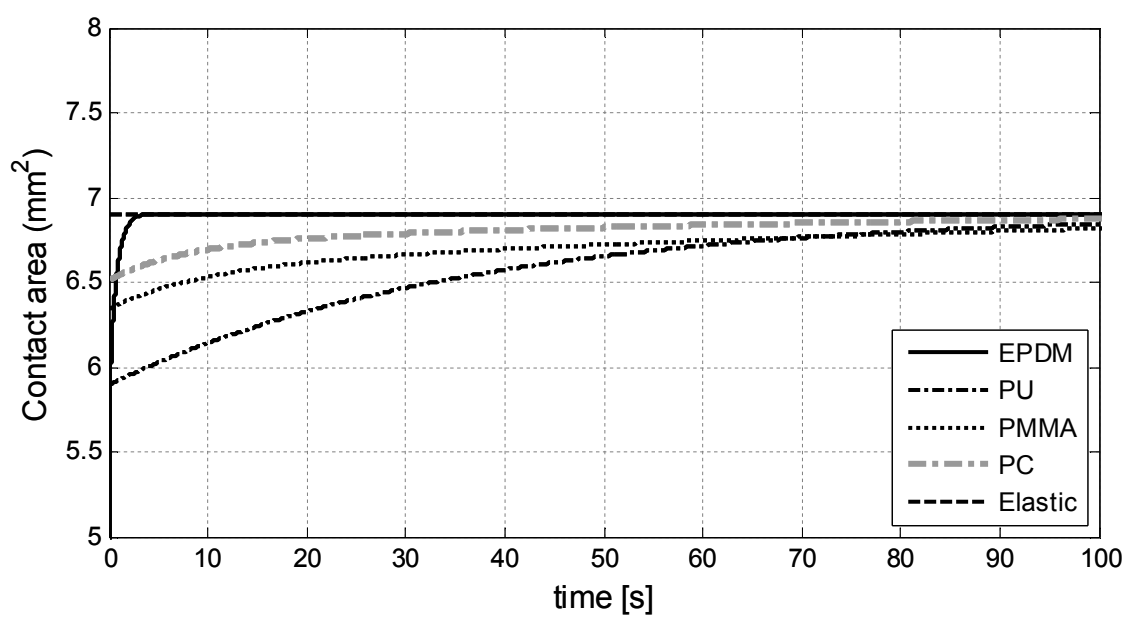

Fig. 4.12: Influence of normalized creep compliance on the contact area.

It can be concluded that the relevance of a viscoelastic contact model is greatly dependent on the time scale in which the contact occurs in relation with the characteristic retardation times of the viscoelastic material.

\subsubsection{Influence of elastic modulus in the plane of indentation $\left(E_{x}\right)$}

To study the influence of one of the elastic modulus on the contact area, the effect of reinforcement in the plane normal to the indentation direction is shown in Fig. 4.13. The reinforcement is assumed as an increase of the elastic modulus in one direction $(x)$ of the indentation plane $(x, y)$ without changing the rest of the elastic parameters, shear moduli or Poisson's ratios. In Fig. 4.13 a), the black solid line shows the contact area for the isotropic case and the dashed lines show the contact area at various reinforcements of $E_{x}$. It can be seen that due to the reinforcement the contact area decreases slightly with increasing $E_{x}$. Fig. $4.13 \mathrm{~b}$ ) shows the ratio between the axes of the contact area, in which unity means a circular contact area. It can be seen that due to reinforcement in $E_{x}$, the contact area has a different shape: from circular in the isotropic case, the contact area becomes increasingly elliptical with increasing $E_{x}$, the anisotropic case. 
a)

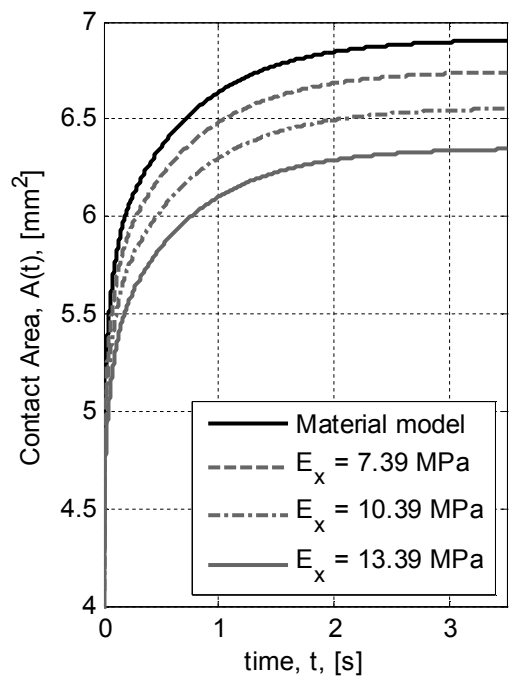

b)

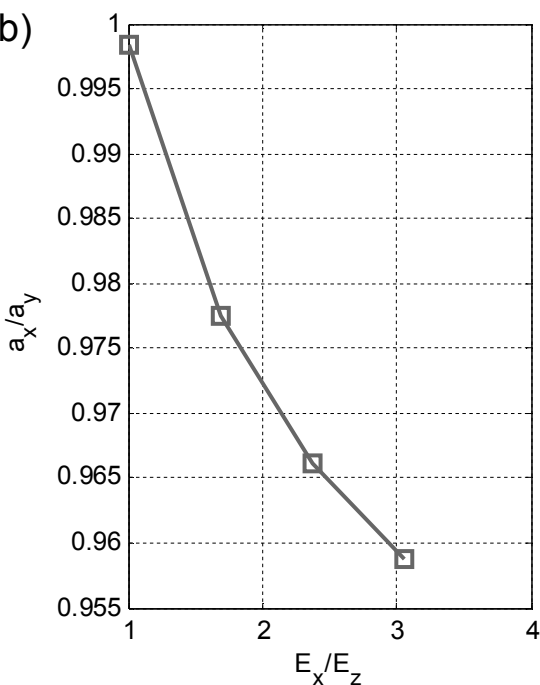

Fig. 4.13: a) shows the influence of increasing elastic modulus in one direction of the indentation plane on the contact area, b) shows the ratio between the radius of the contact at different degrees of reinforcement.

Fig. 4.14 shows the influence of each shear modulus on the contact area. The shear moduli in different directions are varied from being constant to be dependent of $E_{x}$, as $G=E_{x} /(2(1+v))$, therefore increasing with an increase of the reinforcement. 
a)

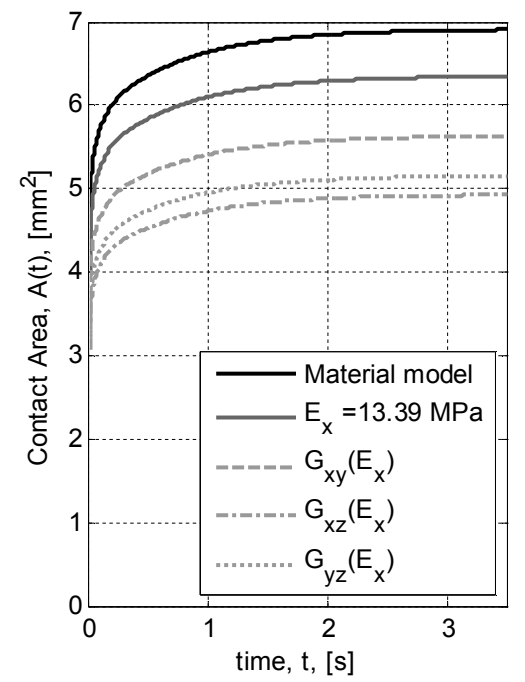

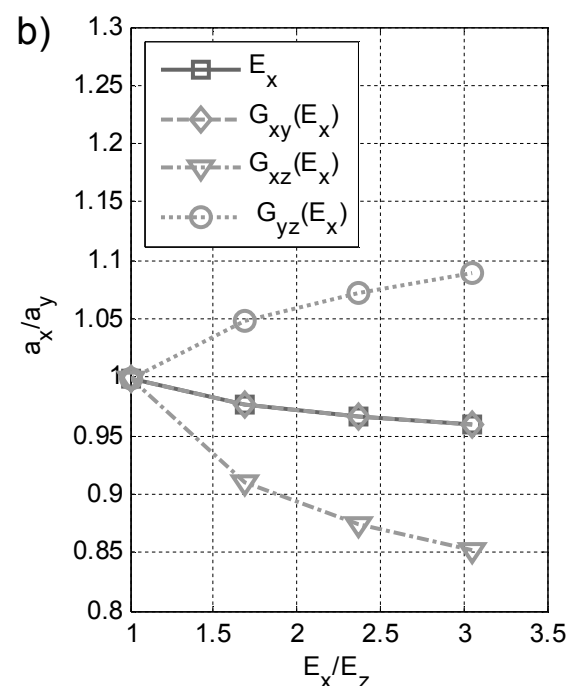

Fig. 4.14: a) shows the influence of increasing the shear modulus on the contact area, b) shows the influence of increasing the shear modulus on the ratio between the principal radii of the contact area.

In Fig. $4.14 \mathrm{a}$ ) it is shown that an increase of the shear moduli causes a significant decrease of the contact area. Fig. 4.14 b) shows that the modification of shear modulus affects the ratio of the principal axes of the contact area, but the shape of the contact area remains elliptical.

\subsubsection{Influence of elastic modulus in the direction of indentation $\left(E_{z}\right)$}

Fig. 4.15 shows the influence of the elastic modulus of the material in the direction of indentation on the contact area. The isotropic case is shown by a solid black line, and increasing $E_{z}$ is represented by dashed lines. In Fig. $4.15 \mathrm{a}$ ) it can be seen that the contact area decreases significantly with increasing the elastic modulus in the direction of indentation $\left(E_{z}\right)$. This effect is larger that of increasing $E_{x}$, as seen in Fig. 4.13. The increase of the elastic modulus in the direction of indentation, as shown in Fig. 4.15 b), does not have an effect on the shape of the contact area, which remains circular. 
a)

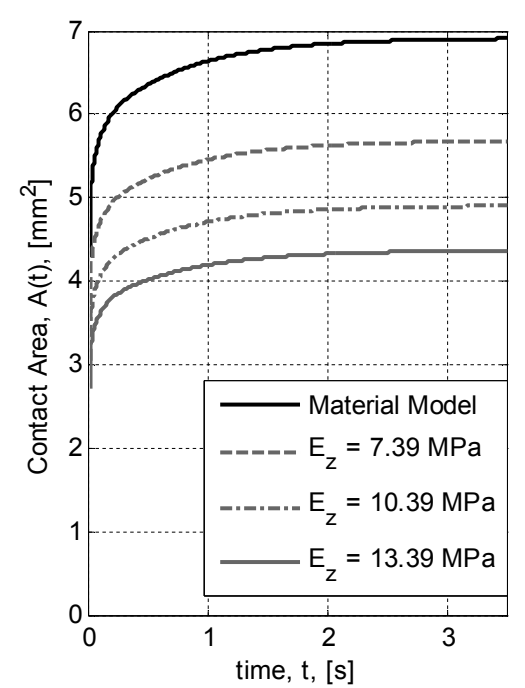

b)

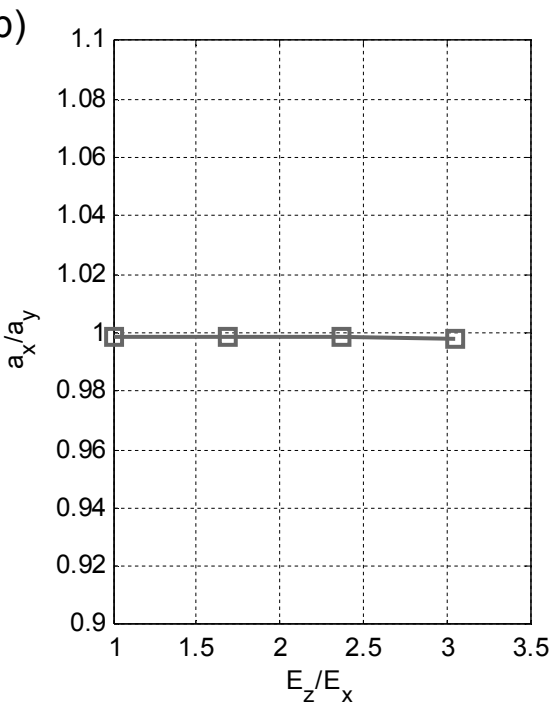

Fig. 4.15: a) shows the influence of increasing $E_{z}$ on the contact area, b) shows the influence of increasing $E_{z}$ on the ratio of the principal radii of the contact area.

a)

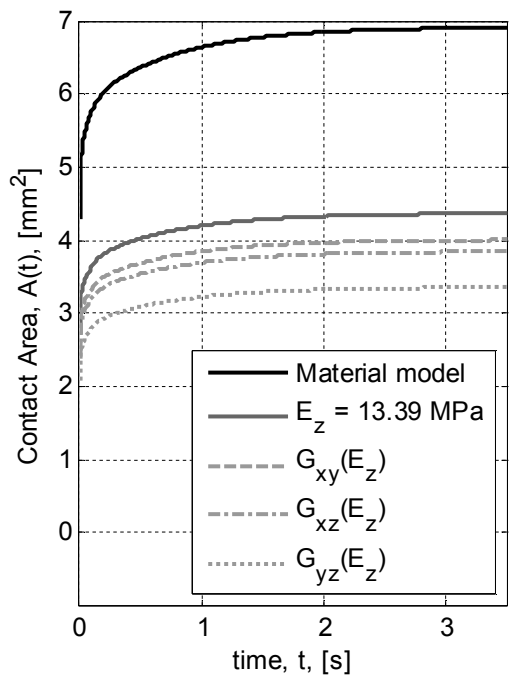

b)

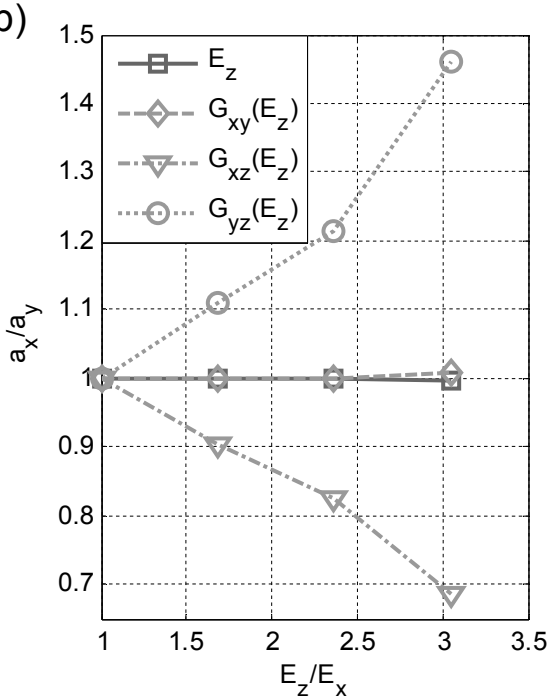

Fig. 4.16: a) shows the influence of different shear modulus in the contact area, b) shows the influence of shear modulus on the ratio of the principal axes of the contact area. 
In Fig. 4.16 a), it is shown that an increase of any of the shear modulus causes a decrease of the contact area. In the different dashed lines each shear modulus varies as $G=E_{z} /(2(1+v))$, whilst the other shear modulus remain constant. Each shear modulus affects the contact area with different magnitudes depending on the direction of the shear modulus, as seen in Fig. $4.16 \mathrm{~b}$ ). Different directions of shear modulus can affect the shape of the contact area, from circular to elliptical, as seen in Fig. 4.16 b).

\subsubsection{Comparison with viscoelastic contact models available in literature for the isotropic case}

The radius of the contact area calculated with the orthotropic viscoelastic isochronous contact model (OVI) is compared with the results obtained using the viscoelastic contact model of Ting [14]. For an isotropic material and a controlled load case, in which the normal load follows a Heaviside step function, Ting's radius of the contact area is defined as:

$$
a(t)=\left[\frac{3 R F_{N}}{8} \phi(t)\right]^{1 / 3}
$$

A comparison between the developed orthotropic viscoelastic isochronous (OVI) contact model with the isotropic viscoelastic contact model of Ting [14] was made using the elastic and viscoelastic material parameters measured for EPDM (presented in the section Material Model), the results are shown in Fig. 4.17.

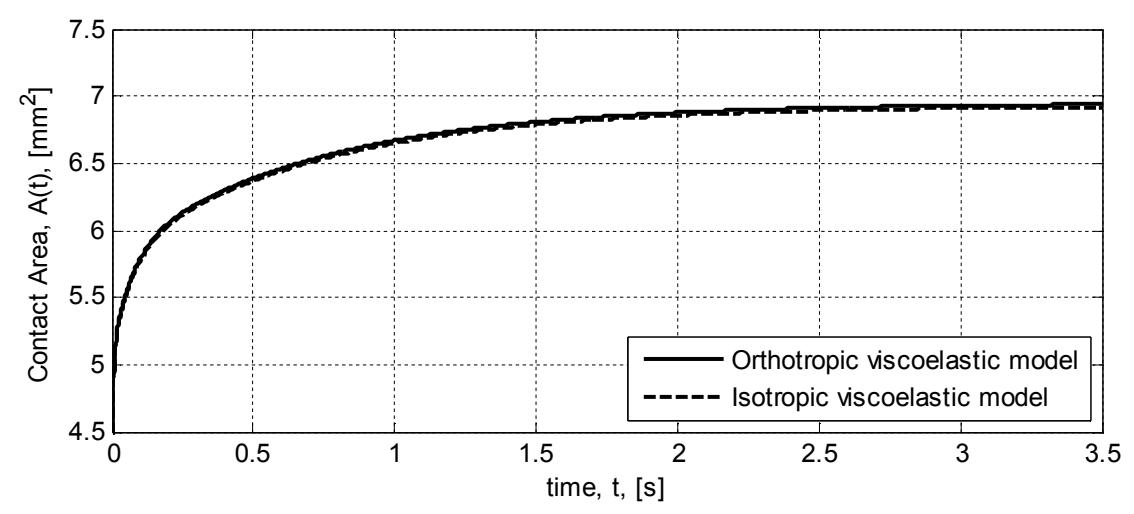

Fig. 4.17: Comparison of the orthotropic viscoelastic isochronous contact model with the isotropic viscoelastic contact model of Ting [14].

The contact area shows a small difference of $0.35 \%$ between the two models, which is a result of the input parameters of the orthotropic 
viscoelastic isochronous contact model which are more numerous and determined with different types of tests than the single input parameter of the Ting model, and therefore a slight difference of results is expected. It is concluded that, when used for isotropic materials, the results of the orthotropic viscoelastic anisotropic contact model agree with the results found in literature.

\subsubsection{Viscoelastic Anisotropy}

To study the viscoelastic anisotropic contact model an analysis of a composite made of FR-4 epoxy matrix reinforced with 7628 woven glass fabric is made. The anisotropic elastic properties of this composite have been described by Hutapea and Grenestedt [55], shown in Table 4.2, while Shrotriya et al. [56] characterized the viscoelastic properties, shown in Table 4.3.

The time dependent contact area was calculated using these material parameters, assuming that the direction of indentation is normal to the warp and woven direction, the results are shown in Fig. 4.18.

Table 4.2: Elastic properties of woven glass/epoxy composite [55].

\begin{tabular}{cccccc}
\hline$E_{x}$ (warp) GPa & $E_{y}$ (Fill) $(\mathrm{GPa})$ & $G_{x y}(\mathrm{GPa})$ & $G_{x z}(\mathrm{GPa})$ & $G_{y z}(\mathrm{GPa})$ & $v$ \\
\hline 21.95 & 21.8 & 3.52 & 1.84 & 1.72 & 0.08 \\
\hline
\end{tabular}

Table 4.3: Retardation times and compliant coefficients for woven glass/epoxy, in the warp direction [56].

\begin{tabular}{cccccc}
\hline $\mathrm{i}$ & $\phi_{i}(\mathrm{GPa})$ & $\lambda_{i}(\mathrm{~s})$ & $\mathrm{i}$ & $\phi_{i}(\mathrm{GPa})$ & $\lambda_{i}(\mathrm{~s})$ \\
\hline$\phi_{r}$ & $1.26 \cdot 10^{-1}$ & & 5 & $1.32 \cdot 10^{-2}$ & $6.89 \cdot 10^{5}$ \\
1 & $3.81 \cdot 10^{-3}$ & $1.61 \cdot 10^{1}$ & 6 & $1.33 \cdot 10^{-2}$ & $6.06 \cdot 10^{6}$ \\
2 & $5.48 \cdot 10^{-3}$ & $5.55 \cdot 10^{2}$ & 7 & $1.55 \cdot 10^{-2}$ & $1.00 \cdot 10^{8}$ \\
3 & $2.29 \cdot 10^{-3}$ & $9.09 \cdot 10^{3}$ & 8 & $1.05 \cdot 10^{-2}$ & $5.49 \cdot 10^{8}$ \\
4 & $5.62 \cdot 10^{-3}$ & $6.25 \cdot 10^{4}$ & 9 & $1.57 \cdot 10^{-2}$ & $5.00 \cdot 10^{9}$ \\
\hline
\end{tabular}




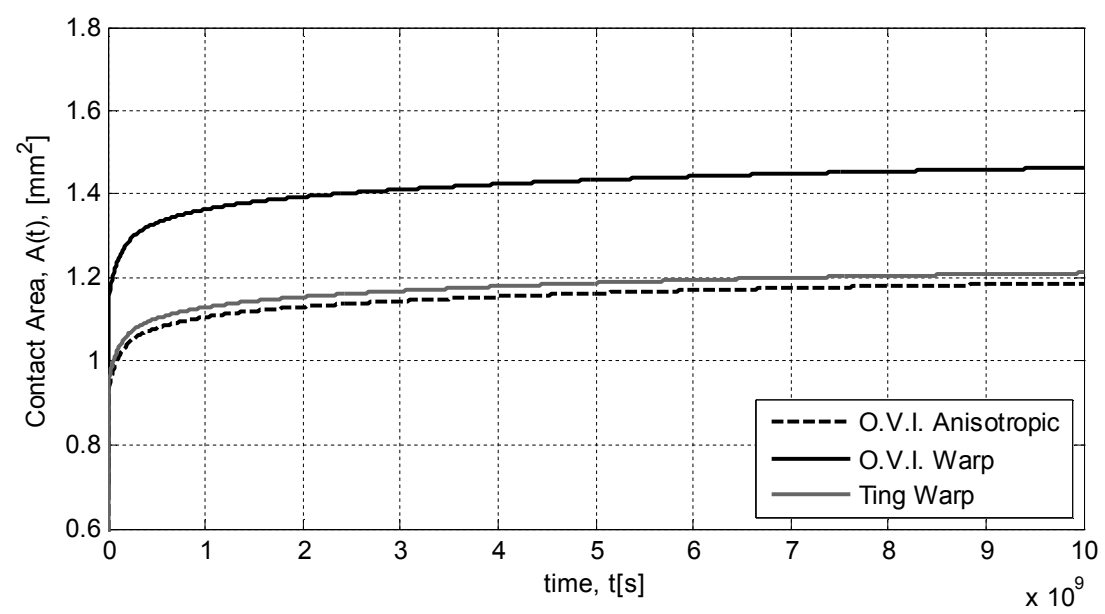

Fig. 4.18: Contact area in woven glass epoxy composite.

In Fig. 4.18 the contact area of a woven glass epoxy composite was calculated considering a spherical indenter with $5 \mathrm{~mm}$ radius and a normal load of $1 \mathrm{kN}$. The contact area was calculated under three configurations:

- The anisotropic case of the developed viscoelastic contact model, by using the elastic material parameters enlisted in Table 4.2 and the normalized creep compliance defined by the parameters in Table 4.1. Shown by the solid black line.

- The isotropic case of the developed viscoelastic contact model, by using the elastic material parameters corresponding to the warp direction, $E=21.95 \mathrm{GPa}$ and $G=3.52 \mathrm{GPa}$ and the normalized creep compliance. Shown by the solid grey line.

- The isotropic case by using a unidirectional viscoelastic contact model, like Ting's, with input parameters the (non-normalized) creep compliance function of the composite in the warp direction, defined by the parameters in Table 4.1. Shown by the dashed black line.

The difference between the anisotropic contact area, shown as a solid black line, and the contact area defined by the creep compliance in the warp direction, shown as a dashed black line, is $23 \%$. This is explained by the great influence that the shear modulus in each direction has on the calculation of the contact area. An isotropic viscoelastic contact model, with material properties of the reinforced material, will predict lower contact areas than an orthotropic viscoelastic contact model, because it will 
overestimate the shear modulus of the material, here corresponding to the properties of the warp direction. To show that the $23 \%$ higher contact area calculated in the anisotropic case (solid black line) is caused by an overestimation of the shear modulus, the contact area of an isotropic material defined with the properties of the warp direction (indentation direction) was calculated using the orthotropic viscoelastic isochronous contact model, i.e. the input parameters are elastic and viscoelastic.

The difference of the contact area between the isotropic case in the warp direction and the unidirectional viscoelastic model of Ting is of $2 \%$. It is concluded that the contact area calculated with a viscoelastic isotropic contact model, used with the properties of an anisotropic material, overestimates the material properties, obtaining a too small contact area.

\subsubsection{Conclusions}

A contact model for viscoelastic orthotropic materials was developed by separating the time and spatial variables. This contact model is able to consider the influence of different material properties in the principal directions $(x, y, z)$ and the influence of the viscoelastic behaviour of the material by using one normalized creep compliance function, therefore the viscoelastic behaviour is modelled isochronously.

The normalized creep function influences the contact area due to the retardation times, without modifying the magnitude of the contact area. The influence of varying the elastic modulus in one direction of the plane of indentation was studied. It was found that the contact area decreases only slightly but that an additional increase of the shear modulus causes it to decrease significantly. Varying the elastic modulus in the direction of indentation (increase of $E_{z}$ ) causes a significant decrease of the contact area, and by varying the shear modulus the shape of the contact area can vary from circular to elliptical, depending on the direction of the shear modulus. The viscoelastic orthotropic isochronous contact model was compared with results from literature, by using the data of an isotropic material and the parameters of an anisotropic one. Both comparisons were in line with those existing in literature and with the parameterization study of the previous section.

\subsubsection{Discussion}

The addition of fibres to an elastomer causes anisotropy, especially in the direction in which the fibres are aligned. However, due to the short length of the fibres is difficult to align them, therefore composites with short fibres often show low degrees of anisotropy. This means that the material 
properties are different in each direction, but the difference is rather low. On the other hand, the shear modulus of an elastomeric composite material is influenced mainly by the shear modulus of the matrix in each direction. As the elastomer is isotropic the shear modulus of the composite will behave very similarly to the elastomer and will not be greatly influenced by the direction of the fibres.

Dynamic mechanical analysis (DMA) was performed on EPDM without fibres and on EPDM reinforced with 5 phr of Twaron $®$ aramid fibres, oriented unidirectionally. The DMA analysis was performed in shear mode, with the fibres aligned longitudinally to the shear plane $(L)$. The procedure is explained in Section 3.1.2 and the device is shown in Fig. 3.2. The results of these measurements are the storage and loss modulus in shear of the EPDM and the fibre reinforced EPDM, as seen in Fig. 4.19.

The creep compliance was calculated using the method explained in Appendix F. With the creep compliance of the EPDM and fibre reinforced EPDM it is possible to calculate the contact area by using the unidirectional anisotropic viscoelastic contact model. The results are shown in Fig. 4.20.

a)

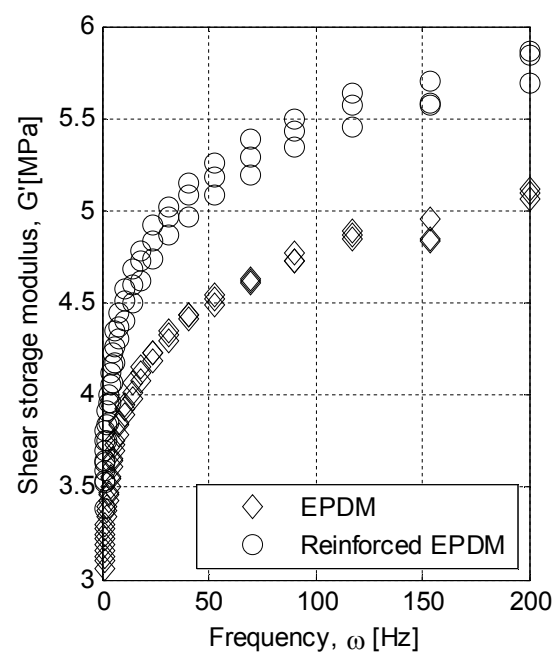

b)

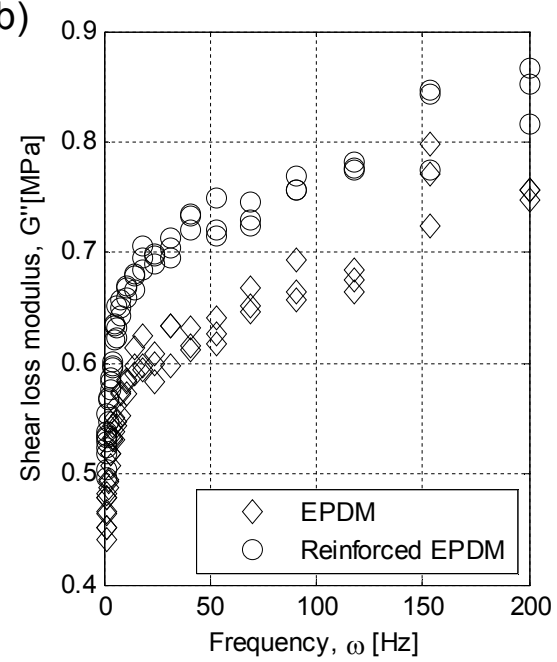

Fig. 4.19: a) shows the shear storage modulus of EPDM and $5 \mathrm{phr}$ fibre reinforced EPDM, b) shows the shear loss modulus of EPDM and 5phr fibre reinforced EPDM. 
a)

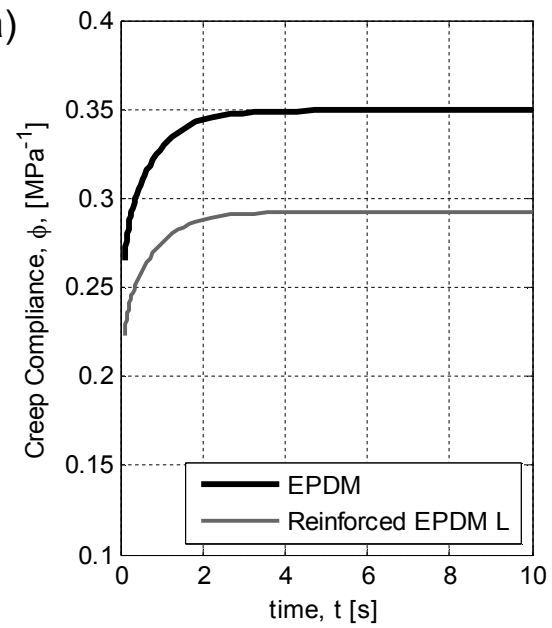

b)

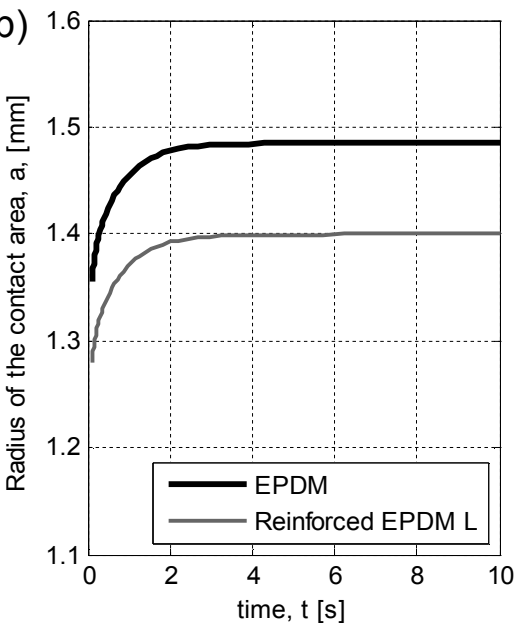

Fig. 4.20: a) shows the creep compliance of EPDM and reinforced EPDM. b) shows the radius of the contact area.

In Fig. 4.20 a), the contact area was calculated considering a spherical indenter with radius $R=12.5 \mathrm{~mm}$ and a normal applied load given by $F_{N}=2 \mathrm{~N}$. The creep compliance of EPDM is approximately $20 \%$ higher than the creep compliance of reinforced EPDM. The radius of the contact area considering EPDM is approximately 6\% higher than in the case of reinforced EPDM. It can be seen that the differences in material properties are rather small, and therefore the differences between the calculated contact areas are even smaller. With such small differences of material properties, the unidirectional contact model offers a good approximation of contact area. Greater differences of the creep compliance are expected in the case of fibres that are aligned normal to the shear plane.

As the orthotropic viscoelastic isochronous contact model requires nine elastic material parameters plus a creep compliance function, it is concluded that the unidirectional viscoelastic contact model, developed from an extension of Sun et al. [17,18], offers the best compromise of good results and ease of obtaining the material parameters.

The orthotropic isochronous contact model allows a qualitative analysis of the contact behaviour of the composite material, by assuming certain material properties. Considering that the shear modulus remains rather constant at different amounts of reinforcement, the contact area in a viscoelastic material with fibres oriented along a direction $(x$ or $y)$ in the 
plane of indentation will be larger than the contact area in a viscoelastic material with fibres oriented normal to the plane of indentation $(z)$.

\subsection{The dynamic contact area}

As mentioned in Section 2.2.4.1, for viscoelastic materials the contact area calculated in the static case differs in shape and in size to the contact during sliding. A recent study by Arvanitaki et al. [25] showed a reduction of the contact area and a change in the shape of the contact area with increasing velocity. They show, in Fig. 4.21, the mean diameter of the contact area as a function of the sliding velocity, for an indenter of $8 \mathrm{~mm}$ radius, an elastomer of elastic modulus $E=1.16 \mathrm{MPa}$ and under different applied loads.

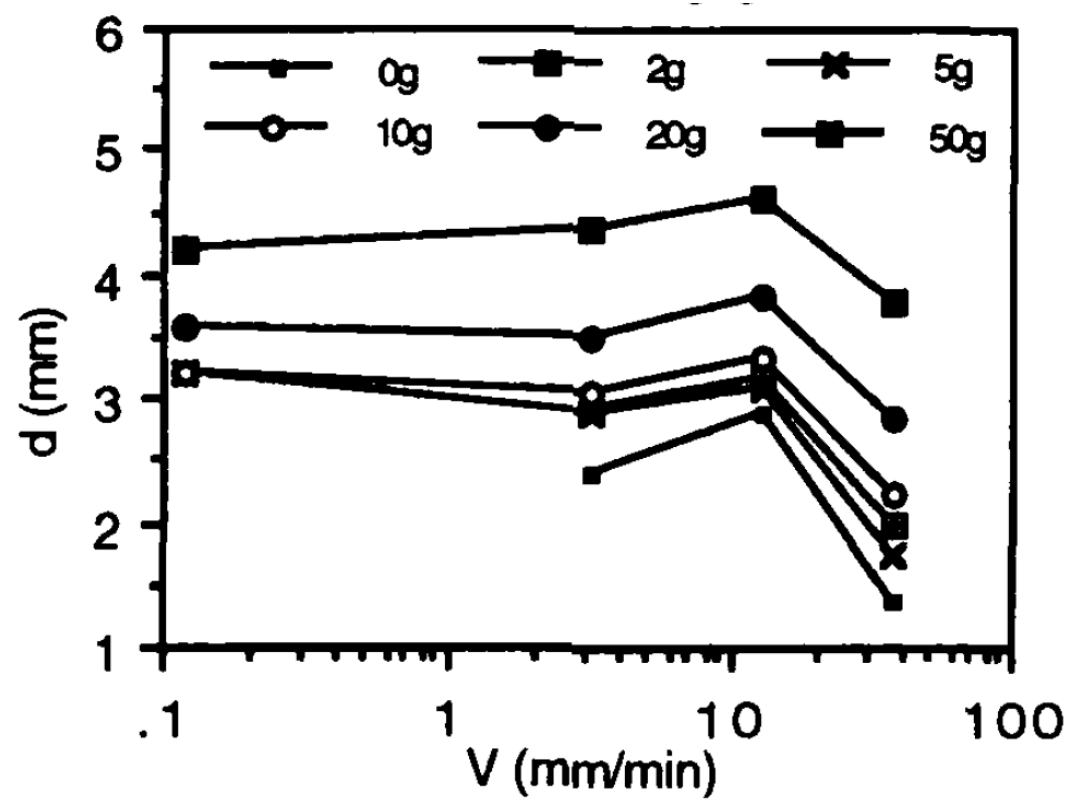

Fig. 4.21: Contact diameter as a function of sliding speeds, at different applied normal loads [25].

The tribo-system studied in this thesis considers various loading conditions and sliding velocities in the order of $0.2 \mathrm{~m} / \mathrm{s}$, therefore it is necessary to measure the contact area and determine how the contact area changes with time. 


\subsubsection{Experiments}

Sliding experiments between a rubber spherical indenter and a smooth glass plate were performed. The normal applied load was set to $F_{N}=2 \mathrm{~N}$ and the radius of the elastomeric indenter was $R=3 \mathrm{~mm}$. A camera was fitted normal to the plane of contact and pictures of the contact area were taken at different sliding speeds. Fig. 4.22 a) shows the contact area in the static case. It can be seen that the contact area has a circular shape. Fig. $4.22 \mathrm{~b}$ ) shows the contact area at a sliding speed of $0.11 \mathrm{~m} / \mathrm{s}$. It can be seen that the shape of the contact area changes when sliding takes place, the contact area becomes elliptical with the major semi-axis normal to the sliding direction.
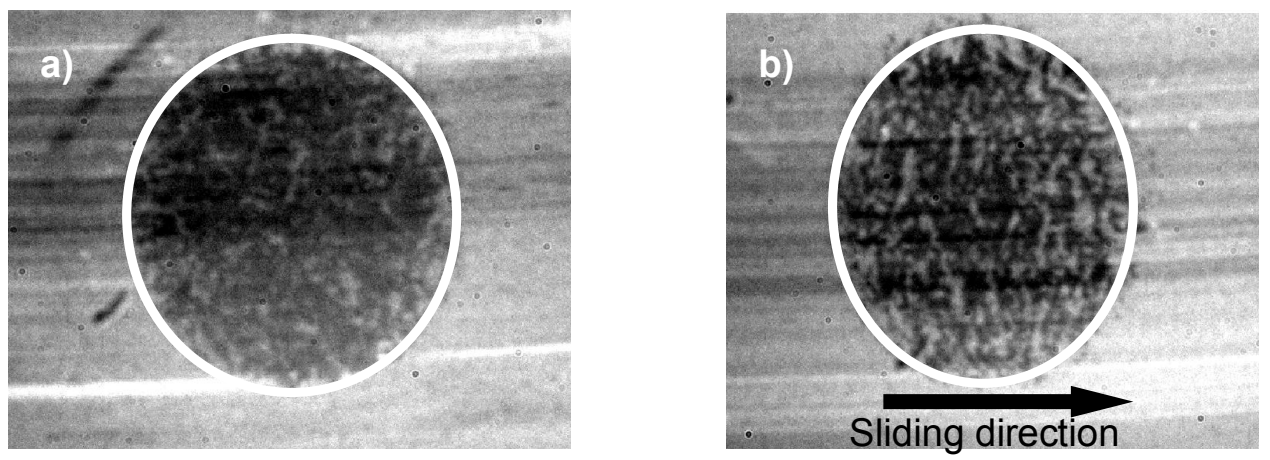

Fig. 4.22: Photographs of the contact area between an elastomeric spherical indenter and a glass disk, a) in static contact, b) in sliding contact at $0.11 \mathrm{~m} / \mathrm{s}$.

The contact area was measured in the static case and for seven sliding velocities: $0 \mathrm{~mm} / \mathrm{s}, 3.6 \mathrm{~mm} / \mathrm{s}, 15.2 \mathrm{~mm} / \mathrm{s}, 24.8 \mathrm{~mm} / \mathrm{s}, 70.5 \mathrm{~mm} / \mathrm{s}, 118.2$ $\mathrm{mm} / \mathrm{s}, 249.8 \mathrm{~mm} / \mathrm{s}$ and $638 \mathrm{~mm} / \mathrm{s}$. An average of 10 measurements of the contact area were taken at each sliding speed; the results are shown in Fig. 4.23, allowing a qualitative comparison with the results of Arvanitaki et al. [25] shown in Fig. 4.21. In Fig. 4.23, the measured contact area at various sliding speeds is shown. As the plot has a logarithmic scale, the static contact is plotted at a sliding speed of $10^{-5} \mathrm{~m} / \mathrm{s}$. 


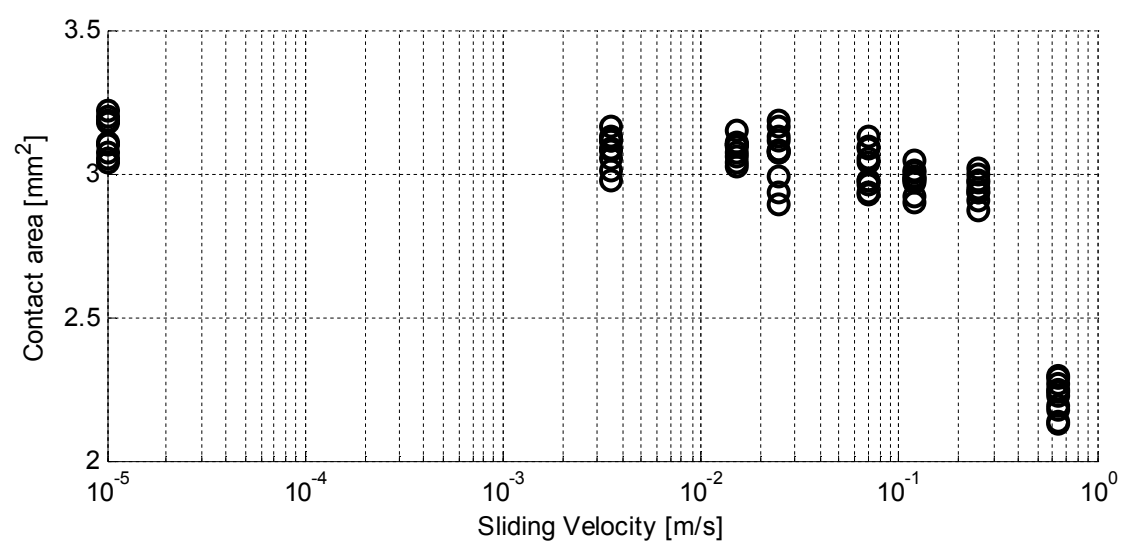

Fig. 4.23: Measured contact areas at different sliding speeds, between an elastomeric spherical indenter and a glass plate.

The change of the contact area with sliding speed can be fitted in its dimensionless form with an arctan-shaped curve, see Eq. 4.36. Using the normalized average values obtained in the static case and the seven sliding speeds results in the curve fit shown by the dotted black line in Fig. 4.24.

The contact area is fairly constant with sliding velocity until $0.2 \mathrm{~m} / \mathrm{s}$, after which it sharply drops with increasing sliding velocity. With the experimental setup used, sliding speeds above $0.64 \mathrm{~m} / \mathrm{s}$ are not possible, so no data points could be obtained beyond this value. However, it is unlikely that the contact area would become zero for very high speeds, it rather approaches a limit. Due to the relation $A \sim E^{-2 / 3}$, Ludema and Tabor [21] suggested that the contact area depends on the sliding velocity in a similar form as the material properties of an elastomer depend on the frequency of deformation. The viscoelastic material properties of the EPDM elastomer used in this thesis, were measured in a Dynamic Mechanical Analysis (DMA) in order to obtain material parameters similar to the stiffness of an elastic material (more details are given in Appendix F). The ratio between the minimum coefficient of relaxation strength, $G_{1}$, and the strength at equilibrium, $G_{e}$, (values shown in Table 3.1) allows obtaining a minimum limiting value for the contact area at high sliding velocities, $v>>1 \mathrm{~m} / \mathrm{s}$. This is the ratio between the modulus at high and at low deformation rate and is given by:

$$
\frac{G_{1}}{G_{e}}=\frac{0.83 \mathrm{MPa}}{2.86 \mathrm{MPa}} \approx 0.3
$$


Therefore the contact area at very high sliding velocities is about 0.3 times the static contact area: $A_{v>>1 \mathrm{~m} / \mathrm{s}}=0.3 A_{\text {static }}$. With this constraint at very high velocities, the fit represented by the dashed black line is obtained.

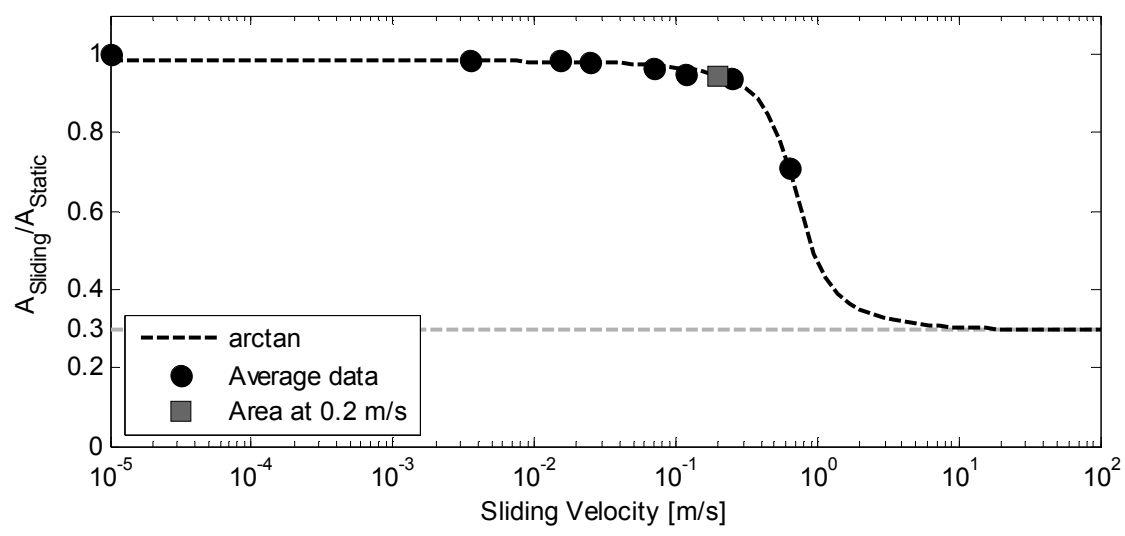

Fig. 4.24: Dimensionless contact area at different sliding velocities.

$$
\frac{A_{\text {sliding }}}{A_{\text {static }}}=a \arctan (b v+c)+d
$$

The fitting parameters of this equation are given in Table 4.4.

Table 4.4: Fitting parameters of Eq. 4.36.

\begin{tabular}{cc}
\hline & Fit $\mathrm{A}_{\text {final }} / \mathrm{A}_{\text {static }}=0.3$ \\
\hline $\mathrm{a}$ & 0.25 \\
$\mathrm{~b}$ & -3.46 \\
$\mathrm{c}$ & 2.24 \\
$\mathrm{~d}$ & 0.70 \\
\hline
\end{tabular}

\subsection{Summary}

Two static contact models have been developed and a comparison of these models was presented. It was found that the anisotropy of fibre reinforced elastomer is greatly influenced by the shear modulus in different directions in addition to the elastic modulus in the direction of indentation. The addition of short fibres in an elastomer has only a slight influence on the 
shear modulus of the material, the dynamical measurements performed in shear mode show differences of less than $20 \%$ between EPDM with and without fibres. Therefore the unidirectional viscoelastic contact model gives a good estimation of the size of the contact area, requiring only one viscoelastic parameter. A qualitative study of the contact behaviour of an anisotropic material can be obtained using the orthotropic viscoelastic isochronous contact model, considering that the shear modulus is hardly influenced by the fibres. This model predicts that the smallest contact area is obtained with fibres oriented along the indentation direction, then with fibres in the plane of indentation and the biggest contact area is found for an unreinforced elastomer.

The change in contact area during sliding is experimentally investigated and an arctan function is proposed to describe the contact area during sliding based on the afore mentioned static contact model. 



\section{Chapter 5 Shear stresses in the contact}

Early investigations into the sliding friction of elastomers showed an important influence of the interface of the contact, triggered by the interaction between the surfaces in contact $[22,57,58]$. More recently Ghatak et al. [59] and She et al. [60] pointed out that adhesion, friction and fracture can be described by the kinetics occurring in the interface of a tribosystem formed by an elastomer. Grosch [33] performed a very complete experimental study where he measured the effects of velocity, temperature and surface roughness on friction. He concluded that there are two mechanisms which contribute to the friction between an elastomer and a hard counter surface: adhesion and energy losses arising from the deformation of the rubber at the contacts with the hard surface asperities. He related both factors to the material properties of the rubber bulk. These examples illustrate the different approaches followed to describe the origin of elastomeric friction, either focused on the viscous behaviour of the bulk elastomer, or focused on the adhesion in the interface of the contact. In Chapter 3 the importance of these two mechanisms of friction, i.e. deformation and adhesion, in the present tribo-system was studied. Based on those results, in this thesis the friction behaviour is mainly attributed to the adhesion mechanism of friction, determined as the product between the normal projection of the real contact area and the shear stresses between the contacting surfaces.

The shear stresses between the contacting surfaces relate to the breaking of the weakest attractive bonds that are created between the surfaces. These bonds can be within the bulk of one of the contacting materials and in that situation the shear stresses in the bulk surface are responsible for the adhesive friction, or these bonds can be in the interface between the contacting bodies and then the shear strength of the interface determines the adhesive friction.

To obtain the shear stresses that occur in the contact area during sliding friction, it is necessary to characterize the properties of the interacting surfaces. Previous publications $[45,46]$ suggest possible modifications to surfaces of elastomers as the result of sliding friction, with the modification of the surface layer being responsible for differences in the tribological behaviour of the studied systems. This means that if a modification of the surface occurs due to friction, it should be taken into account. 
A recent publication by Lorenz et al. [61] attributed part of the measured friction of an elastomer sliding against a concrete surface to the shearing of the real contact area. They identified a smear film created during the running-in period of the friction experiments and in the presence of this film, friction originates from the shearing of this contamination layer. In the present chapter, the appearance of such an interface modification and the influence on friction is studied. Furthermore, the behaviour of the shear stress developed in the interfacial layer due sliding contact is modelled. With this model, it is possible to calculate the friction force and friction coefficient.

\subsection{Development of surface layer due to friction}

To analyze the existence of a surface layer in tyre-road contact, the cross section of the tread of a passenger car tyre was examined with Scanning Electron Microscopy (SEM). The SEM analysis was performed in vacuum, without sputter coating in a Carl Zeiss 1550 HRSEM using two types of detectors, secondary electron detector (SE2) and InLens detector. The SE2 detector is a conventional detector of secondary electrons and the InLens detector is a more efficient detector, especially suitable for obtaining high resolution images at low voltages.

In Fig. 5.1 the cross section of the passenger car tyre tread is shown. The upper part of the image shows the tyre surface which used to be in contact with the road and the bottom part of the image shows the rubber bulk of the tyre tread. It can be seen that the edge between the cross section and the surface is distinguished by a bright line, which appears different from the bulk.

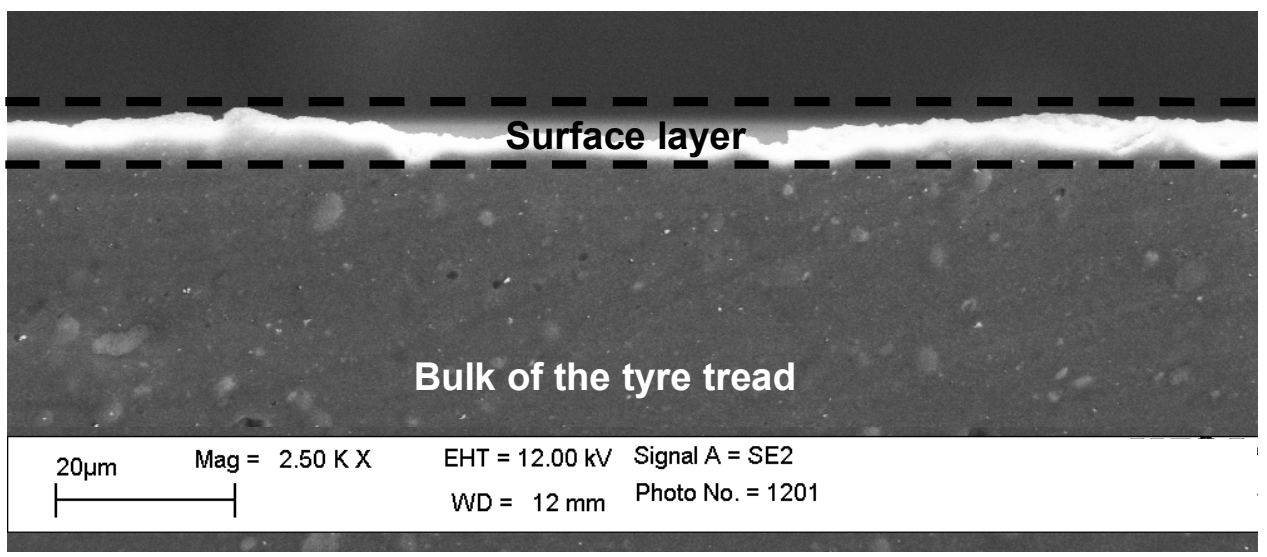

Fig. 5.1: SEM image of the cross section of a passenger car tyre tread. 
Fig. 5.2 shows a higher magnification of the cross section. Clusters of randomly distributed particles of carbon black can be seen in the bulk. Towards the edge of the cross section with the surface, the distribution of these particles becomes more compact, apparently merging until no individual particles can be observed anymore.

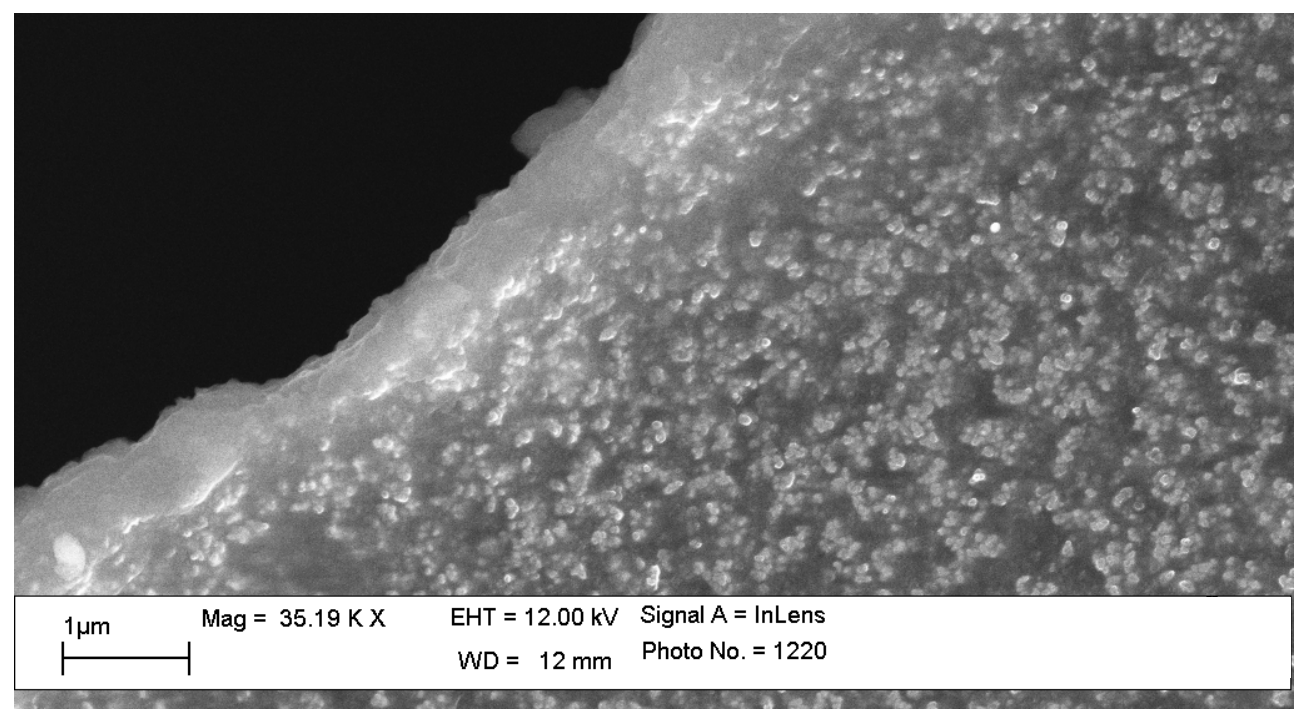

Fig. 5.2: SEM image of the cross section of the tread, at higher magnification.

\subsubsection{Controlled tribo-generation of surface layers}

To determine the development of this surface layer in the present tribological system in a more reproducible and controlled manner, sliding friction experiments were performed using a pin-on-disk tribometer. The disks are made of EPDM, see Appendix $\mathrm{C}$, which has high homogeneity and consistency of properties, limiting material variations between the experiments. The pin is made of $100 \mathrm{Cr} 6$ steel, with radius $R=12.5 \mathrm{~mm}$. The applied load is $2 \mathrm{~N}$ and the sliding velocity is $0.2 \mathrm{~m} / \mathrm{s}$, ensuring a contact pressure and a sliding velocity similar to the tyre-road contact in steady state. The friction experiments were performed at a controlled temperature of $23^{\circ} \mathrm{C}$ and a humidity of $60 \%$. The test ran for a sliding distance of $500 \mathrm{~m}$, meaning that the running-in period was finished and a rather constant friction coefficient was obtained. During the running-in phase the interfacial layer between the contacting surfaces develops and reaches a steady state. 


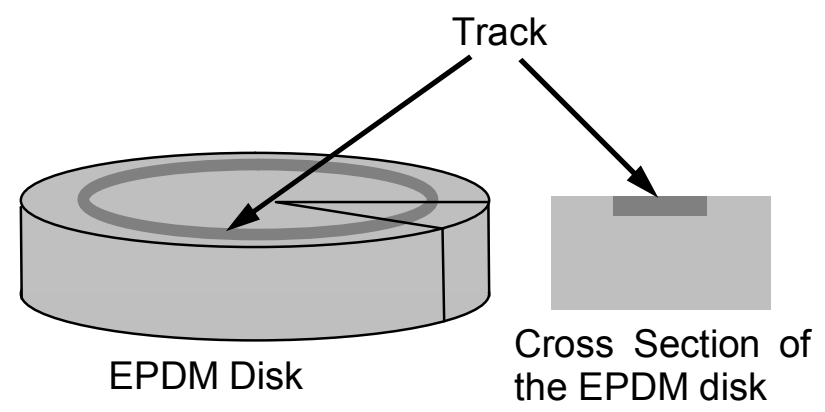

Fig. 5.3: Sample taken from an EPDM disk, after performing friction experiments; the wear track of the sample can be observed in the cross section.

To be able to observe the thickness of the interfacial layer after the sliding friction experiments, a cube of $5 \mathrm{~mm} \times 5 \mathrm{~mm} \times 5 \mathrm{~mm}$ was obtained from the cross section of the rubber disk, as shown in Fig. 5.3. The disk was cut at ambient temperature with a scalpel and then analysed in the SEM, without prior coating.

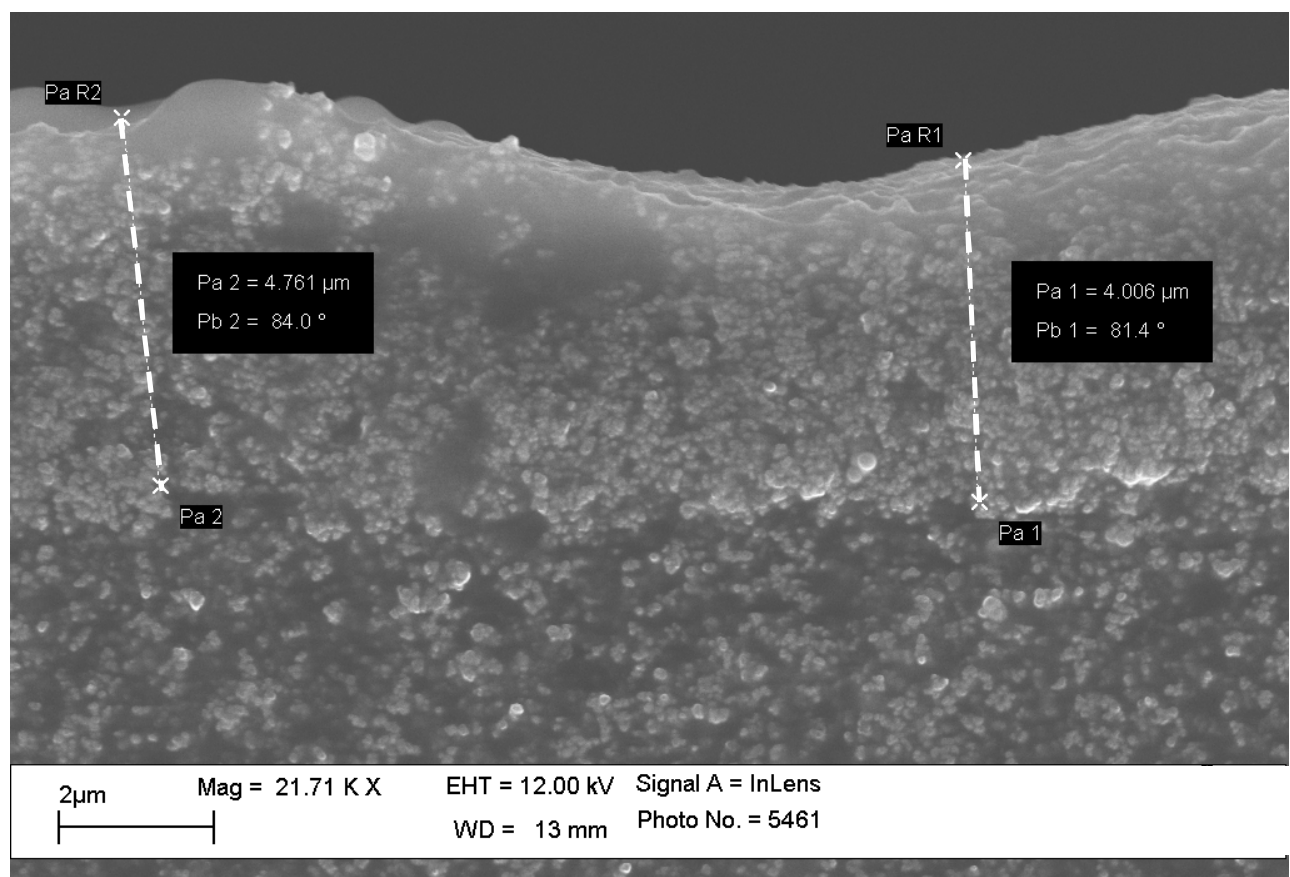

Fig. 5.4: SEM image of the cross section of the EPDM track, in the vicinity of the surface. 
Shear stresses in the contact

Fig. 5.4 shows the SEM image of the cross section of the EPDM disk, at high magnification and in the vicinity of the surface. It can be seen that compactly arranged clusters of particles are present in the vicinity of the surface, while towards the bulk these clusters are arranged more spaciously. This is very similar to the structure previously observed on the tread of a used passenger tyre, Fig. 5.2. This confirms that the structure of the surface changes due to sliding contact.

\subsubsection{Roughness measurements of the tribo-generated surface layer}

Surface roughness will not only influence the tribological properties of the layer, but also has to be taken into account when analysing the mechanical properties and the contact behaviour of the surface.

Surface roughness measurements were performed employing a Keyence Color 3D Laser Confocal Microscope on both the original surface and the tribo generated surface layer. For the roughness measurements, a cut-off length of $120 \mu \mathrm{m}$ was selected, corresponding to the size of the contact area when using a spherical indenter with a radius $R=1 \mathrm{~mm}$ and an applied normal load $F_{N}=2 \mathrm{mN}$. Roughness measurements were performed on seven different spots for each type of surface; the average roughness for the original surface is $R_{a}=0.07 \pm 0.02 \mu \mathrm{m}$ and the average roughness for the tribo-generated surface layer is $R_{a}=0.19 \pm 0.06 \mu \mathrm{m}$.

a)

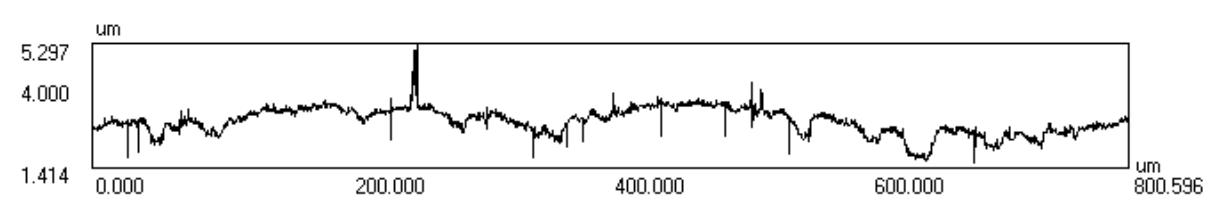

b)

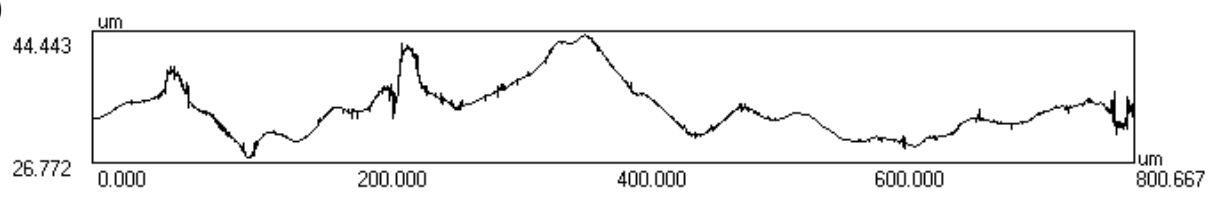

Fig. 5.5: In a): roughness results of an area of the original surface. $\ln b$ ): roughness results of an area of the tribo-generated surface layer, both measurements are shown at a sampling length of $800 \mu \mathrm{m}$.

\subsubsection{Application to contact mechanics}

Hertz's theory for elastic contact assumes perfectly smooth surfaces. Greenwood, Johnson and Matsubara [62] defined a non-dimensional 
roughness parameter, $\alpha$, defined in Eq. 5.1, which indicates if any effects of surface roughness have to be taken into account. For values of $\alpha$ less than 0.05 , the effect of the roughness in the contact is negligible and the contact can be considered to follow Hertz theory [62].

$$
\alpha=\frac{R_{a} R}{a^{2}}
$$

In which $R_{a}$ indicates the surface roughness, $R$ refers to the radius of the indenter and a to the contact radius.

Inserting the measured surface roughness values gives $\alpha=0.019$ for the original surface, and $\alpha=0.047$ for the tribo-generated surface layer. This means that for the measured surfaces in contact with a spherical indenter with a radius of $1 \mathrm{~mm}$ at an applied load of $2 \mathrm{mN}$, any surface roughness effects on the contact behaviour can be neglected and the Hertzian theory can be used to describe the contact area.

\subsubsection{Application to indentation measurements}

From Fisher-Cripps [63] it is known that high surface roughness can produce larger uncertainties when using depth indentation measurements to estimate the mechanical properties of the surface. It is recommended to perform indentation at a depth of at least twenty times the surface roughness, $\delta>20 \times R_{a}$, to have an uncertainty of less than $5 \%$. Therefore using the same contact conditions, $R$ and $F_{N}$, the indentation in the original surface should be at least $\delta=1.4 \mu \mathrm{m}$ and in the tribo-generated surface layer should be at least $\delta=3.8 \mu \mathrm{m}$.

\subsubsection{Mechanical properties of the tribo-generated surface layer}

To study whether the tribo-generated surface layer also has altered mechanical properties, indentation experiments were performed on the original surface and on the surface layer using a Shimadzu Micro Hardness tester. Tweedie and Van Vliet [64] studied nanoindentation of viscoelastic materials and concluded that linear viscoelastic theory cannot be used to interpret indentation experiments performed with sharp indenters. For spherical indenters with a sufficiently large radius the maximum contact pressure is lower than the elastic strain limit, enabling the indentation to be represented using linear viscoelastic theory. Following the conclusions in [64], the Shimadzu Micro Hardness tester was equipped with a spherical indenter with a radius of $1 \mathrm{~mm}$. To minimize energy dissipation trough 
Shear stresses in the contact

viscous response of the elastomer, the maximum loading rate allowed by the instrument was used [64].

Indentation measurements were performed on the original EPDM surface and on the surface layer at a range of maximum normal loads, allowing the effect of the EPDM substrate on the indentation measurements of the surface layer to be identified. Fig. 5.6 shows the load-indentation curve at three different loads for each surface. Each curve is composed of a loading phase, a holding load phase and an unloading phase. The holding phase lasted $500 \mathrm{~ms}$ in all measurements. Each curve represents the average of three measurements, the average standard deviation for the original surface is 0.012 and for the surface layer it is 0.041 . It can be noticed that the indentation depths of the measurements are more than twenty times the surface roughness, ensuring reproducible results [63].

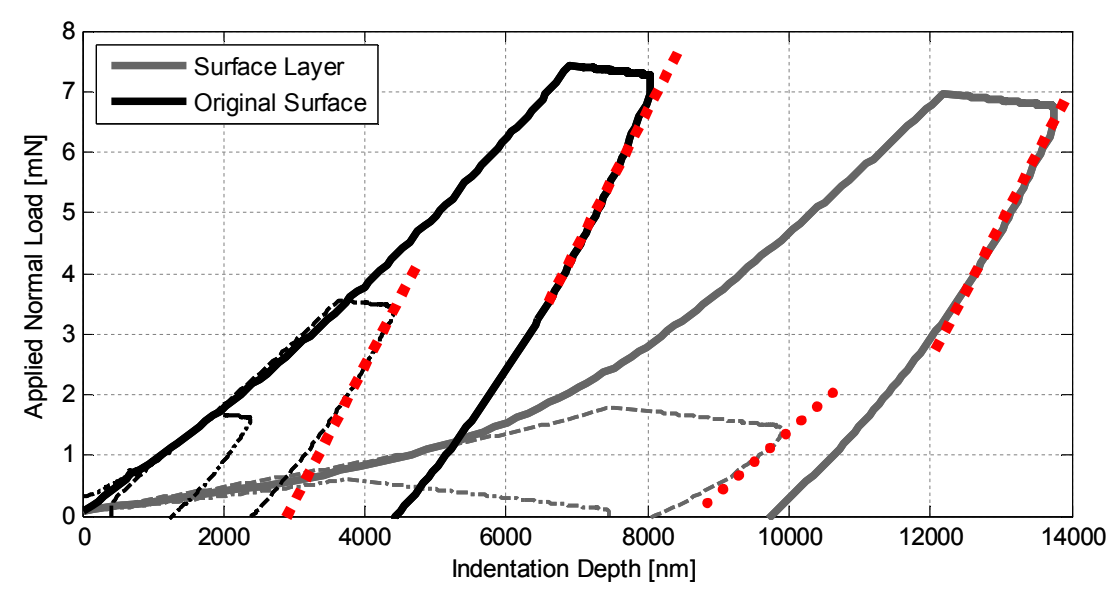

Fig. 5.6: Load-Indentation curve for both surfaces, with a holding time of $500 \mathrm{~ms}$.

In Fig. 5.6 the black lines show the results obtained on the original surfaces and the grey lines show those for the tribo-generated surface layer. It can be seen that the measurements obtained on the surface layer show a significantly higher indentation depth than those on the original surface, meaning that the surface layer has a low stiffness.

An estimate of the Young's modulus can be obtained from the value of the slope of the unloading curve, Oliver and Pharr [65], as indicated by the segmented lines. For the original surface, at all three normal loads, a similar slope can be seen. For the surface layer the same slope is obtained only at the highest normal load; at lower normal loads the unloading curve 
has a lower slope. From Fig. 5.6 it can be concluded that the surface layer has different material properties than the bulk.

To quantify the differences of properties between the original surface and the surface layer, the creep compliance was calculated from the variation on indentation depth during the holding time, $\delta(t)$, in Fig. 5.6 using the solution of Ting [14] and the relation of Tweedie and Van Vliet [64] between indentation and creep. The creep compliance in shear under spherical indentation is given by:

$$
\phi(t)=\frac{8 \sqrt{R}}{3 F_{N}}[\delta(t)]^{3 / 2}
$$

The resulting creep compliances are shown in Fig. 5.7. The various creep compliances obtained for the original surface are also compared with the creep compliance as determined using DMA measurements. These results are shown in Fig. 5.7 a), where it can be seen that all the creep functions obtained by indenting the original surface are rather similar. There is a small difference between the curves obtained at different loads, but this variation falls within the range usually observed for mechanical measurements on elastomers. In Fig. 5.7 b) the creep compliance functions obtained by indenting the generated surface layer are shown. It can be seen that at low normal loads the creep compliance is much higher than at high loads. This shows that the surface layer has rather poor material properties in comparison with the bulk. 
a)

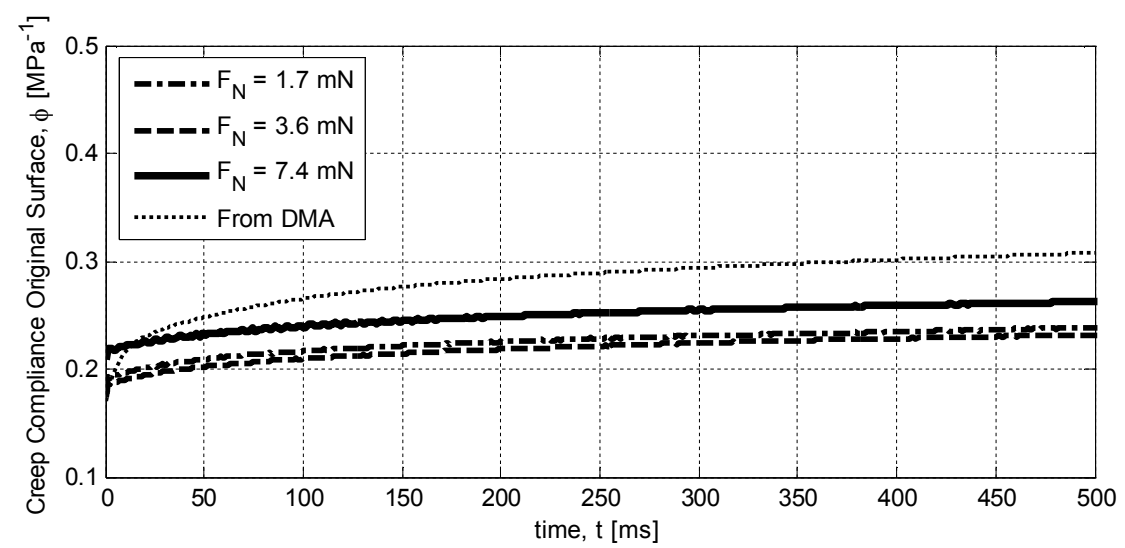

b)

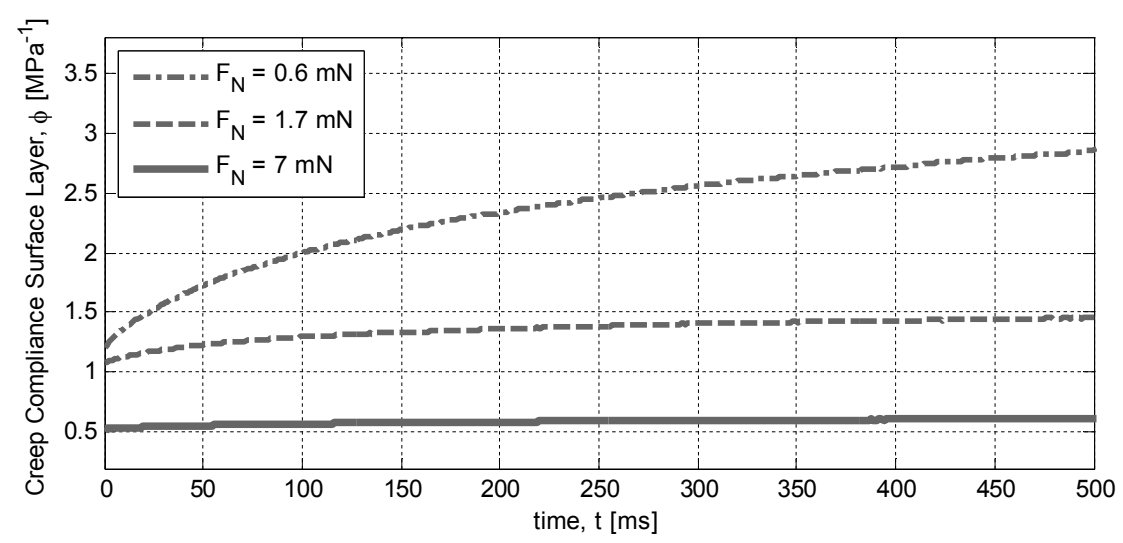

Fig. 5.7: Creep Compliance at different normal loads: on a) the original surface and on b) the tribo-generated surface layer.

In order to quantify the differences between the properties of the EPDM bulk and the properties of the surface layer, the creep compliance function at $0.6 \mathrm{mN}$ was fitted with Eq. 4.1 describing a series of exponential terms, similar to Prony series in a stress relaxation measurement. The results are given in Table 5.1. They show an increase of three orders of magnitude in the relaxed creep compliance, $\phi_{r}$, and a much slower behaviour. 
Table 5.1: Compliance coefficients and retardation times of the EPDM Bulk and of the generated surface layer.

\begin{tabular}{ccccc}
\hline & \multicolumn{2}{c}{ Bulk EPDM } & \multicolumn{2}{c}{ Surface Layer } \\
\hline $\mathrm{i}$ & $\phi_{i}\left(\mathrm{MPa}^{-1}\right)$ & $\lambda_{i}(\mathrm{~s})$ & $\phi_{i}\left(\mathrm{MPa}^{-1}\right)$ & $\lambda_{i}(\mathrm{~s})$ \\
$\phi_{r}$ & $3.49 \mathrm{E}-01$ & & $6.40 \mathrm{E}+02$ & \\
1 & $4.69 \mathrm{E}-02$ & 0.0064 & $1.82 \mathrm{E}-01$ & 0.0073 \\
2 & $4.90 \mathrm{E}-02$ & 0.0713 & $9.16 \mathrm{E}-01$ & 0.108 \\
3 & $8.23 \mathrm{E}-02$ & 0.7284 & $6.34 \mathrm{E}+02$ & 499.7 \\
\hline
\end{tabular}

From these results it can be concluded that the material properties of the surface layer are significantly reduced in comparison with the properties of the original EPDM surface and bulk.

\subsubsection{Influence of the tribo-generated surface layer on the tribological behaviour of the system}

To study the differences in frictional behaviour between the tribo-generated surface layer and the original surface, sliding friction experiments were performed on the original surface and on the surface layer with a pin-ondisk tribometer and with a nano-tribometer using reciprocal motion.

The samples with a surface layer were prepared by sliding a smooth spherical indenter over the elastomer in a pin on disk tribometer over a distance of $500 \mathrm{~m}$ at a sliding velocity of $0.2 \mathrm{~m} / \mathrm{s}$, allowing the surface layer be fully developed. Following this, a friction experiment was performed using the same pin-on-disk setup, at sliding velocities of $0.003 \mathrm{~m} / \mathrm{s}, 0.03$ $\mathrm{m} / \mathrm{s}$ and $0.2 \mathrm{~m} / \mathrm{s}$. In Fig. 5.8 these measurements are compared with the measurements obtained on samples without a pre-generated layer; it can be seen that for all sliding velocities, the coefficient of friction measured on the pre-formed surface layer is the lowest. 


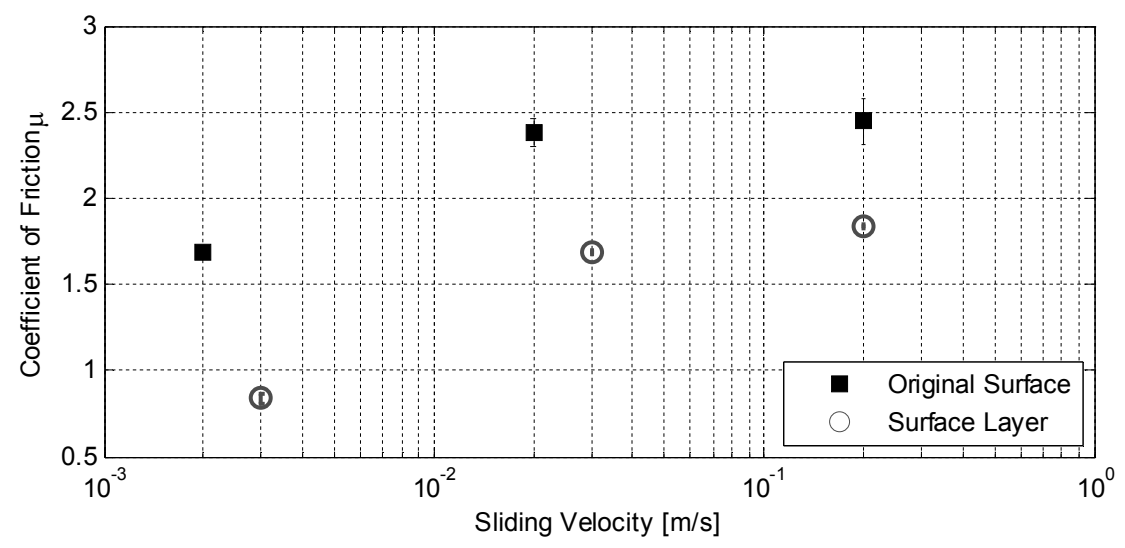

Fig. 5.8: Coefficient of friction measured by performing friction experiments on the original surface and on the pre-generated surface layer.

\section{Nano-tribometer experiments}

The differences in tribological performance between the original material and the samples with a tribo-generated surface layer may be studied in more detail at low loads. Executing experiments at lower loads and lower indentation depths ensures that the sliding contact will be more influenced by the tribo-generated surface layer and less by the bulk, and employing a Nanotribometer (CSM Instruments, Switzerland) allows this. The contact geometry was a ball-on-flat configuration, using a $100 \mathrm{Cr} 6$ steel ball with a radius of $1 \mathrm{~mm}$, a sliding velocity of $10 \mu \mathrm{m} / \mathrm{s}$, a reciprocating amplitude of $0.64 \mathrm{~mm}$ and a normal load of $2 \mathrm{mN}$. The applied normal loads produce an indentation depth into the original surface and in the surface layer of approximately $3 \mu \mathrm{m}$ and $7 \mu \mathrm{m}$ respectively, see Fig. 5.6. Therefore the friction within the track will be influenced by the properties of both the surface layer and the bulk material. Results of these friction experiments are shown in Fig. 5.9: the black line corresponds to friction experiments performed on the original surface and the grey line corresponds to friction experiments performed on the surface layer. 


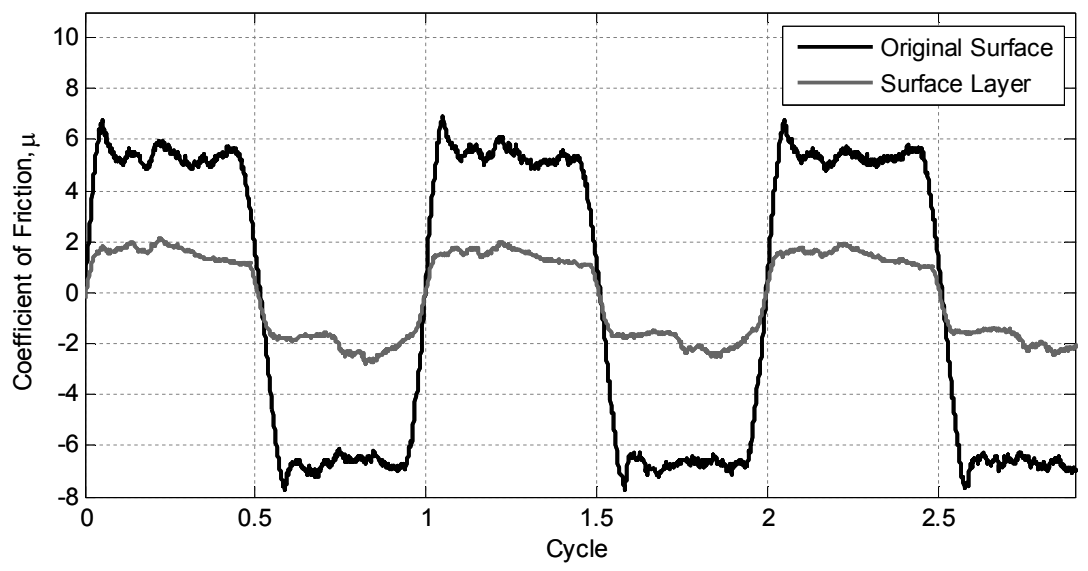

Fig. 5.9: Measured coefficient of friction for the original surface, black line, and the surface layer, gray line, as a function of the number of cycles.

Each measurement consisted of five reciprocating cycles; the two first cycles of each measurement were neglected. An average coefficient of friction was calculated from five measurements with the last three cycles each, for each type of surface. The coefficient of friction measured on the original surface is $\mu=5.97 \pm 0.44$ and on the tribo-generated surface layer $\mu=1.69 \pm 0.60$. The coefficient of friction obtained on the surface layer is much lower than the coefficient of friction obtained on the original surface, agreeing with the trends seen at a low sliding velocity in Fig. 5.8.

The two friction experiments shown in Fig. 5.8 and Fig. 5.9 cannot be quantitatively compared with each other due to differences between the two tribo-systems. Despite that, qualitatively they agree on the large influence of the tribo-generated surface layer on the friction behaviour of the respective tribo-system.

\subsubsection{Discussion}

Quantitative differences were found between the properties of the tribogenerated surface layer and the original surface. The measured material properties and the coefficient of friction on the tribo-generated surface layer are significantly lower than those measured on the original EPDM surface. It has been established in Chapter 3 that in the present thesis, the adhesion mechanism (shearing the adhesion layer) is the prime contributor to friction. According to Bowden and Tabor [21] the friction due to adhesion is given by Eq. 2.11 , by multiplying the shear strength in the interface by the real contact area. As the roughness measurements show that the roughness of 
the two surfaces do not have a significant influence and that the real contact area can be obtained from Hertz theory, Eq. 2.11 can be rewritten and the coefficient of friction due to adhesion follows the shear strength and Young's modulus according to:

$$
\mu_{a} \propto \tau \cdot E^{-2 / 3}
$$

This proportionality means that for a constant shear strength in the interface, a decrease of the elastic modulus results in an increase of the coefficient of friction. In contrast, the obtained experimental results show a decrease in the material properties, whilst the coefficient of friction in the tribo-generated surface layer is lower than in the original surface. It can be concluded that the shear strength of the tribo-generated surface layer is much reduced, acting as a "solid lubricant" within the contact.

The tribologically modified surface shows a considerable decrease in mechanical properties, which strongly affects the frictional behaviour of the tribo-system in which the elastomer operates. Therefore the existence of interfacial layers should be taken into account when analyzing friction with elastomers. In order to describe friction, information regarding the shear strength of the tribo-generated surface layer is a necessity.

\subsection{Modelling the behaviour of the interfacial layer}

As discussed in the previous section, a tribo-generated surface layer, or interfacial layer, can strongly influence the friction behaviour, since friction is caused by shear in the layer close to the contact interface. Therefore it is necessary to understand and model the shear behaviour of this layer. Combined with the known contact area of Chapter 4, this allows the friction to be calculated.

As mentioned previously, the interfacial layer shows a similar behaviour to a solid lubricant layer. Therefore the shear behaviour of the interfacial layer will be modelled using the shear model for lubricated contacts from Popovici [66]. This model considers that the viscoelastic behaviour of the interfacial layer follows a Maxwell model with two elements, and that the shear behaviour of the interfacial layer can be described by models describing the behaviour of boundary lubrication layers [67-69].

A schematic representation of a Maxwell model with two elements can be seen in Fig. 5.10. This model is composed of one spring and one dashpot. The spring represents the shear modulus of the interfacial layer, $G_{L}$, and the dashpot represents the viscosity, $\eta_{L}$, of the interfacial layer. 


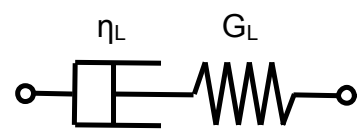

Fig. 5.10: Maxwell two elements model, used to represent the viscoelastic behaviour of the interfacial layer.

The relation between strain and stress of a Maxwell model of two elements is given by Eq. 5.3, where $\tau$ represents the shear stress in the interfacial layer, $\dot{\gamma}$ is the strain rate in shear (shear rate) and $\dot{\tau}$ is the variation of shear stress with time.

$$
\dot{\gamma}=\frac{1}{G_{L}} \dot{\tau}+\frac{\tau}{\eta_{L}}
$$

This strain-stress relation assumes that the material behaves in a Newtonian manner, implying a linear dependency between the shear stresses and the shear strain rate. In sliding contacts the shear rates are often large, which translates in high shear stresses. For lubricants, Bair and Winer [67] observed that at a certain shear stress, the shear stress is limited with further increasing shear strain, this certain shear stress is called limiting shear stress. If the shear stresses in the contact are higher than this limiting shear stress, the shear stresses become constant and the lubricant layer will shear plastically.

The various shear models that describe the behaviour of interfacial layers [67-69] used in this thesis to model the behaviour of the tribo-generated interfacial layer have different nomenclature. To facilitate a comparison, two dimensionless numbers are defined [66]: the dimensionless shear stress parameter in Eq. 5.4 and the dimensionless viscosity parameter in Eq. 5.5.

$$
\begin{gathered}
\bar{\tau}=\frac{\tau}{\tau_{L}} \\
\xi=\frac{\eta_{L} \dot{\gamma}}{\tau_{L}}
\end{gathered}
$$

Where $\tau_{L}$ represents the limiting shear stress, the ratio $\tau_{L} / \dot{\gamma}$ can be interpreted as the limiting viscosity in the contact area. Rewriting Eq. 5.3 with the dimensionless numbers gives Eq. 5.6, where $f(\bar{\tau})$ can be 
regarded as a dissipative function that relates the shear strain rate with the shear stress for the different shear models.

$$
\xi=\frac{\eta_{L}}{G_{L}} \frac{d \bar{\tau}}{d t}+f(\bar{\tau})
$$

A range of models is available to describe the dissipative function $f(\bar{\tau})$. The value of $f(\bar{\tau})$ based on Maxwell behaviour is given by Eq. 5.7. At $\bar{\tau}<1$, the shear stresses are lower than the limiting shear stress, meaning that the material shows Newtonian behaviour and the shear stress will increase linearly with the shear strain rate. At $\bar{\tau}>1$, the limiting shear stress is reached and the shear stress remains constant, meaning the layer will shear plastically.

$$
f(\bar{\tau})=\left\{\begin{array}{lll}
\bar{\tau} & \text { for } & \bar{\tau}<1 \\
1 & \text { for } & \bar{\tau} \geq 1
\end{array}\right.
$$

Other shear models assume that the dissipative function $f(\bar{\tau})$ is best defined by a non-linear function. The shear model by Bair and Winer [67], considers the dissipative function given by Eq. 5.8. Gecim and Winer [68], defined this function as Eq. 5.9. Elsharkawy and Hamrock [69] proposed a more general model, given by Eq. 5.10, that allows similar behaviour to the other models to be obtained, by varying the parameter $n$. For example, $n \approx 1.8$, gives a similar result to Bair and Winer [67] and $n \approx 2.8$ gives a similar result to Gecim and Winer [68].

$$
\begin{gathered}
f(\bar{\tau})=-\ln (1-\bar{\tau}) \\
f(\bar{\tau})=\operatorname{atanh}(\bar{\tau}) \\
f(\bar{\tau})=\bar{\tau}\left(1-\bar{\tau}^{n}\right)^{-1 / n}
\end{gathered}
$$

When a linear dissipative function such as Eq. 5.7 is inserted, the first order differential equation given by Eq. 5.6 can be solved analytically, as was done by Johnson and Roberts [70]. Using a non-linear dissipative function, such as Eq. 5.8 to Eq. 5.10, makes it necessary to use numerical analysis to solve Eq. 5.6. 


\subsubsection{Solving the shear stress of the surface layer}

The governing equation to study the shear stress in the interfacial layer is given by Eq. 5.6. To solve this equation it is necessary to define the boundary conditions and isolate the dependent variables. The related calculations are shown in the following sections.

Initially, both the shear stresses both outside and on the boundary of the contact area are zero. Hence the boundary condition of the problem is defined by Eq. 5.11, where $x$ and $y$ are the spatial variables and for a contact area in the shape of an ellipse, $a_{x}$ (in sliding direction) and $a_{y}$ are the principal axes of the ellipse.

$$
\tau=0 \quad \text { at } t=0, \text { for }\left(\frac{x^{2}}{a_{x}{ }^{2}}+\frac{y^{2}}{a_{y}{ }^{2}}\right) \geq 1
$$

For an interfacial layer that behaves according to Eq. 5.6 and assuming that the sliding contact results in a steady flow of the layer in the interface of the contact, the derivative with respect to time can be rewritten.

$$
\frac{d}{d t}=U \frac{d}{d s}
$$

Where $U=v / 2$ is the mean velocity. Therefore $v$ denotes the spatial derivative with respect to time as $2 d s / d t$, which in the present case corresponds to the sliding velocity. A contact sliding in a direction along one of the principal axes of the contact ellipse allows the position in $x$ to be related to the position in $y$, facilitating the solution of the differential equation. 


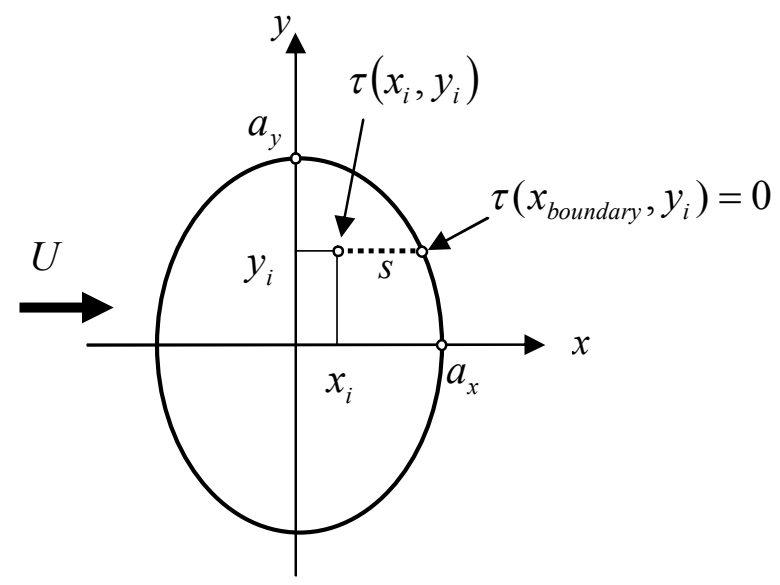

Fig. 5.11: Schematic of the contact ellipse with the assumptions made to calculate the shear stress.

Fig. 5.11 shows a schematic representation of the elliptical contact area, when the sliding direction is given along $x$. The $x_{\text {boundary }}$ is related to the position in the other principal axis as:

$$
x_{\text {boundary }}=a_{x} \cdot \sqrt{1-\frac{y^{2}}{a_{y}^{2}}}
$$

Defining dimensionless axes of the contact ellipse and the maximum value that $X$ can take in the boundary of the contact ellipse as:

$$
X=\frac{x}{a_{x}} ; \quad Y=\frac{y}{a_{y}} \quad \text { and } \quad X_{\text {boundary }}=\sqrt{1-Y^{2}}=\frac{x_{\text {boundary }}}{a_{x}}
$$

The spatial derivative $d s$ can be re-written in the dimensionless form as:

$$
d s=a_{x} d X
$$

Replacing the temporal derivative with the dimensionless spatial derivative from Eq. 5.15, the first order differential equation from Eq. 5.6 is expressed by:

$$
\xi=D \frac{d \bar{\tau}}{d X}+f(\bar{\tau})
$$


Where $D$ is a constant. For lubricants this parameter is known as the Deborah number and is used to characterize the fluidity of a lubricant under certain flow conditions.

$$
D=\frac{\eta_{L} \mathrm{v}}{a G_{L} 2}
$$

To solve the differential equation numerically, the dependent variables need to be isolated. This results in:

$$
\frac{d X}{D}=\frac{d \bar{\tau}}{\xi-f(\bar{\tau})}
$$

Any one-step method for numerical solution of initial value ordinary differential problems can be used to solve Eq. 5.18. Popovici [66] discussed the available numerical solvers for this problem and concluded that the fourth order Runge-Kutta method with fixed step size was the most efficient. This method will also be used in this thesis.

\subsubsection{Parameters required by the shear stress model}

The parameters required to calculate the shear stress in the contact area include:

- Contact area, $A$ : the contact area depends on the contact conditions, the radius of the indenter, the applied normal load and the material properties, as well as on the sliding velocity. To estimate the contact area, the model developed in Chapter 4 is used.

- Sliding velocity, $v$ : the sliding velocity defines the shear strain rate during sliding and influences the Deborah number.

- Thickness of the surface layer, $h$ : together with the sliding velocity, this defines the shear strain rate as $\dot{\gamma}=v / h$.

- Shear modulus of the interfacial layer, $G_{L}$ : this is a property of the surface layer and is obtained from measurements.

- Viscosity of the interfacial layer, $\eta_{L}$ : it is also a property of the surface layer and is obtained from measurements.

- Limiting Shear Stress, $\tau_{L}$ : the limiting shear stress can be related to the elasticity parameter for liquid lubricants as $G_{L} \sim C \tau_{L}[71]$. 
The results of the Maxwell-based shear models presented in Eq. 5.7 to Eq. 5.10 are shown in Fig. 5.12. At large values of the dimensionless parameter $\xi$ all the shear models approach unity, meaning that the shear stress in the contact area equals the limiting shear stress. At low values of the dimensionless parameter $\xi$, the different shear models become important as they show different increases of the dimensionless shear stress with the dimensionless parameter $\xi$. The shear model that describes the behaviour of the layer in the present tribo-system most accurately should include a dependency between the dimensionless shear stress and the sliding velocity, similar to the dependency of friction with sliding velocity in the present tribosystem.

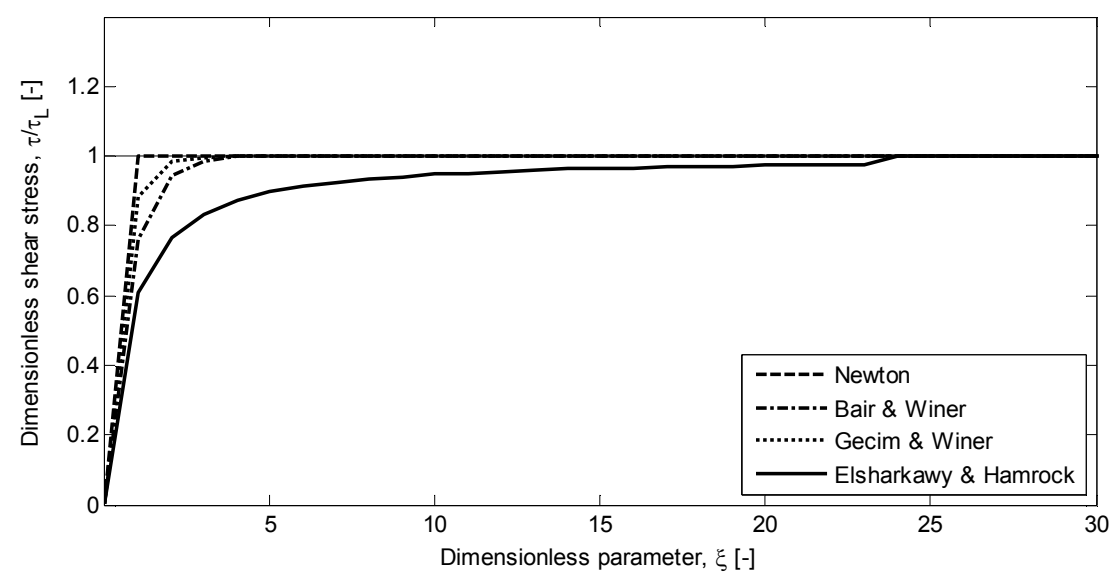

Fig. 5.12 Comparison of the Maxwell based shear models.

To decide the most adequate shear model, the variation of dimensionless shear stress with sliding velocity was compared with the coefficient of friction obtained experimentally at various sliding speeds, with the relation:

$$
\frac{\tau}{\tau_{L}}=\frac{F_{N} \mu_{\text {exp }}}{A \tau_{L}}
$$

Where $F_{N}$ is the normal force, $A$ is the contact area, and $\mu_{\text {exp }}$ is the coefficient of friction obtained from pin-on-disk experiments.

The results are shown in Fig. 5.13. It can be seen that the model by Elsharkawy \& Hamrock, with $n=1.05$, describes the slow increase of dimensionless shear stress with sliding velocity that is in good agreement with the normalized experimental results performed on the surface layer. 
Therefore this model will be used to characterize the shear behaviour in the contact area.

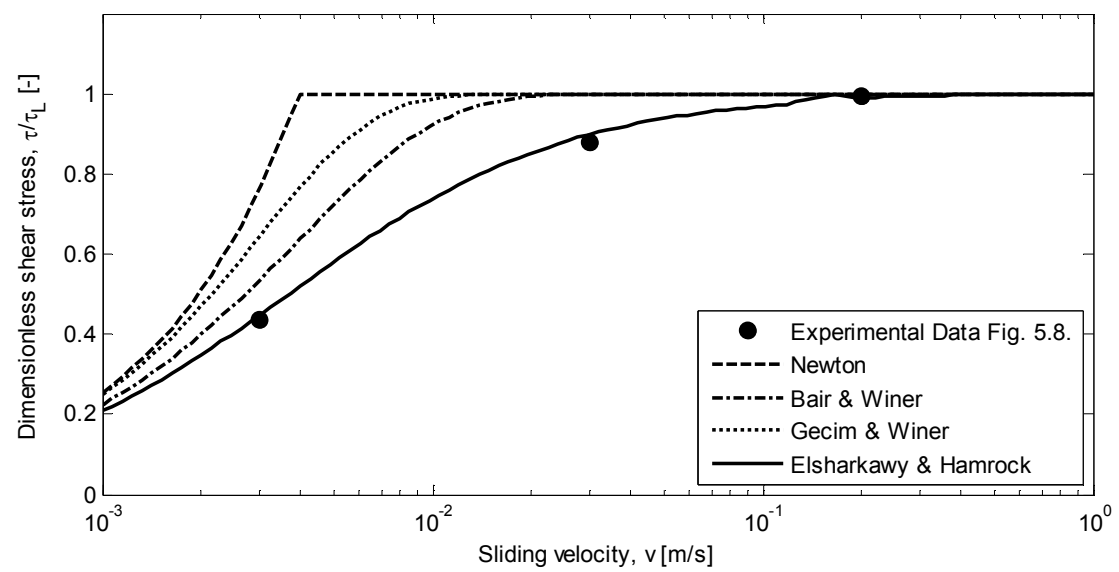

Fig. 5.13: Dimensionless shear stress, depending on the sliding velocity.

A further discussion on how to measure the elastic shear modulus and the viscosity of the layer, as well as how to estimate the limiting shear stress, is given in the following chapter.

\subsection{Summary}

In this chapter, the changes of the surface due to sliding friction were studied. It was found that after repetitive sliding of a steel ball against the EPDM, a surface layer of approximately $4 \mu \mathrm{m}$ thickness is generated. The presence of this layer has a large influence on the friction behaviour of the tribosystem and therefore should be taken into account when analysing friction. Indentation measurements were performed in the surface before and after the sliding experiments, so without and with the tribo-modified surface layer. The results show that the surface layer has lower material properties when compared with the bulk material. In addition, the friction experiments in presence of this layer resulted in a coefficient of friction that is much lower than in its absence. The tribo-generated surface layer behaves similarly to a solid lubricant, the friction being caused by shearing this layer. In order to characterize the friction, the layer is considered to have viscoelastic behaviour, following a Maxwell model, and the shear stress in the layer over the contact area is modelled using existing shear models for interfacial layers [67-69]. From friction experiments performed against a pre-generated surface layer it was possible to approximate the 
behaviour of the dimensionless shear stress at different sliding velocities. After this, the model of Elsharkawy \& Hamrock was found to be the most suitable for describing the shear behaviour of the tribo-generated surface layer. 



\section{Chapter 6 Friction model}

As discussed previously, in the present tribo-system the adhesion mechanism of friction is the most influential. Therefore to describe the friction behaviour, a friction model based on this mechanism has been developed and will be elaborated in this chapter. In Chapter 4 a contact model that considers the anisotropic and viscoelastic behaviour of a composite material indented by a rigid spherical indenter was developed. The contact model developed is valid for static contacts. Sliding experiments were performed to extend this model to include the variation in size and shape of the contact area during sliding. The relation between the static contact area and the sliding contact area can be described by an arctan curve. In Chapter 5, studies of the surface properties and of the modification of the surface due to sliding were performed. It was found that sliding friction results in a modification of the surface properties: a tribogenerated surface layer develops, which is responsible for the friction behaviour of the tribo-system. The shear stresses due to sliding in this tribo-generated surface layer were modelled following models used to describe the shear behaviour of visco-elastic behaving lubricants, considering the viscoelastic behaviour of the tribo-generated layer to follow the two-element Maxwell model.

In the present chapter, the developed contact model and the shear stress model of the tribo-generated surface layer are combined to describe the friction behaviour of the studied tribo-system. A parameter study is included, to observe the importance of the tribo-generated layer. The results are compared with experimental results for the unreinforced and reinforced EPDM.

\subsection{Friction model and parameter study}

The model presented in Chapter 5 gives the behaviour of the shear stress over the contact area. By integrating this over the contact area, the friction force in the contact can be obtained. As the behaviour of the shear stress was calculated numerically, the integration is also performed numerically.

In Eq. 6.1 the dimensionless friction force is expressed in terms of the dimensionless axes $X$ and $Y$ of the contact area as defined in Eq. 5.14: 


$$
\bar{F}=\frac{1}{\pi} \int_{-1}^{1} \int_{-X_{\text {boundary }}}^{+X_{\text {boundary }}} \bar{\tau}(X, Y) d X d Y
$$

The dimensional friction force due to the adhesive mechanism of friction can be obtained by multiplying the dimensionless friction force with the limiting shear stress $\tau_{L}$ and the size of the contact area $A$.

$$
F_{a}=A \tau_{L}\left(\frac{1}{\pi} \int_{-1}^{1} \int_{-X_{\text {boundary }}}^{+X_{\text {boundary }}} \bar{\tau}(X, Y) d X d Y\right)
$$

To calculate the friction coefficient, the total friction force is divided by the normal force, as shown in Eq. 6.3.

$$
\mu_{a}=\frac{A \tau_{L}\left(\frac{1}{\pi} \int_{-1}^{1} \int_{-X_{\text {boundary }}}^{+X_{\text {boundary }}} \bar{\tau}(X, Y) d X d Y\right)}{F_{N}}
$$

As discussed in Chapter 5, the parameters to insert into the model need to be determined. In the model tribo-system studied in this thesis which is used as a reference system, the contact pressure is $0.44 \mathrm{MPa}$, which is obtained using an indenter of radius $R=12.5 \mathrm{~mm}$ and an applied normal load of $F_{N}=2 \mathrm{~N}$. This configuration has been used in the shear stress model, the friction model and the friction experiments. To calculate the contact area, the product between the static contact area and the curve fit obtained for sliding contact was used. The thickness of the surface layer is $4 \mu \mathrm{m}$, as can be seen in SEM images of the cross section of the tribogenerated surface layer, in Fig. 5.4. The elastic shear modulus of the surface layer was determined from the inverse of the creep compliance function found from the microindentation measurements in the surface layer at an applied load of $0.6 \mathrm{mN}$. In Chapter 5 it was seen that the results at an applied load of $0.6 \mathrm{mN}$ show the material properties of the surface layer with the least influence of the substrate. The value for the limiting shear stress is taken as the inverse of the creep compliance function found from the microindentation measurements. The viscosity of the surface layer determines the shape of the $\mu-v$ curve and for a viscosity of $\eta_{L}=580 \mathrm{~Pa} \mathrm{~s}$ the calculated results agree with the experimental results. The contact conditions and the material parameters of the tribo-generated surface layer used in the friction model are shown in in Table 6.1. 
Friction model

Table 6.1: Parameters used in the friction model.

\begin{tabular}{lc}
\hline \multicolumn{1}{c}{ Parameters } & values \\
\hline Applied normal load, $F_{N}$ & $2 \mathrm{~N}$ \\
Static contact area, $A$ & $6.91 \mathrm{~mm}^{2}$ \\
Thickness of the surface layer, $h$ & $4 \mu \mathrm{m}$ \\
Elastic shear modulus of the layer, $G_{L}$ & $0.6 \mathrm{MPa}$ \\
Viscosity of the layer, $\eta_{L}$ & $580 \mathrm{~Pa} \mathrm{~s}$ \\
Limiting shear stress, $\tau_{L}$ & $0.57 \mathrm{MPa}$ \\
\hline
\end{tabular}

In Fig. 6.1 the friction experiments performed in the pre-formed tribogenerated layer are compared with the results from the friction model, for the parameters mentioned in Table 6.1. Each data point of this friction experiments corresponds to 4 measurements. The coefficient of friction at $v=0.003 \mathrm{~m} / \mathrm{s}$ is $\mu=0.84 \pm 0.015$, at $v=0.03 \mathrm{~m} / \mathrm{s}$ is $\mu=1.69 \pm 0.01$ and at $v=0.2 \mathrm{~m} / \mathrm{s}$ is $\mu=1.83 \pm 0.014$.

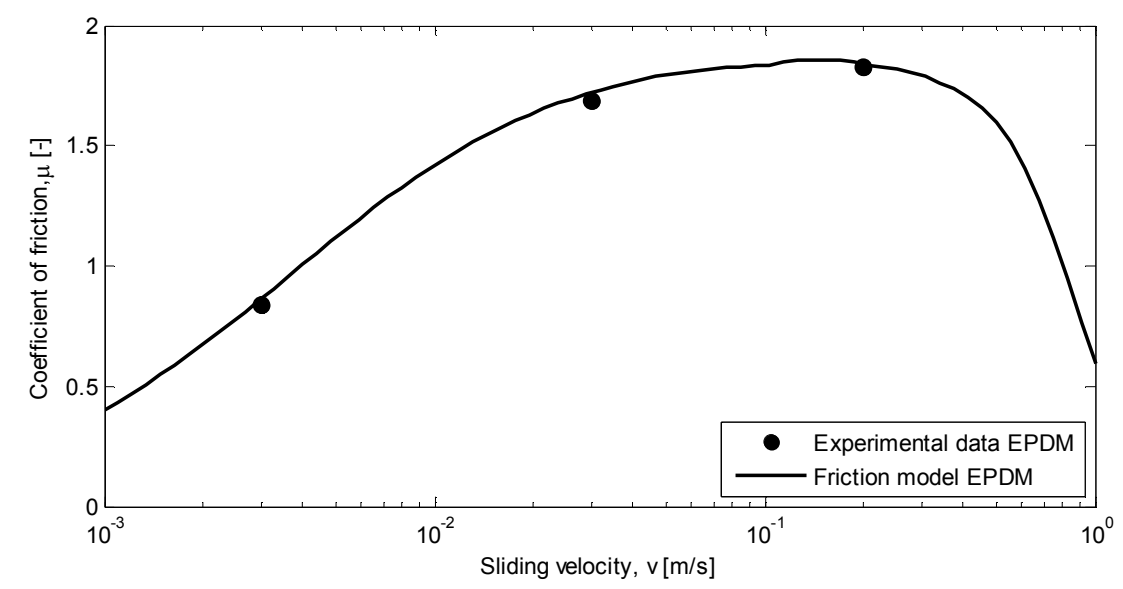

Fig. 6.1: Comparison between the friction model and the friction experiments performed on the tribo-generated surface layer.

It can be seen that by using the parameters shown in Table 6.1, the calculated friction agrees well with the experimental results found on the tribo-generated surface layer. With increasing sliding velocity the coefficient of friction increases from about 0.5 to a fairly constant value of about 1.7 and then drops at sliding velocities above $0.3 \mathrm{~m} / \mathrm{s}$. The coefficient of friction depends on the sliding velocity, because the sliding velocity defines the shear strain rate and therefore the shear stresses in the contact, until the limiting shear stress $\tau_{L}$ is reached. In Fig. 6.1, between sliding velocities 
$v \approx 0.001 \mathrm{~m} / \mathrm{s}$ to $v \approx 0.08 \mathrm{~m} / \mathrm{s}$, the coefficient of friction increases with sliding velocity, due to an increase in the shear stresses in the tribogenerated surface layer. Between the sliding velocities $v \approx 0.08 \mathrm{~m} / \mathrm{s}$ and $v \approx 0.3 \mathrm{~m} / \mathrm{s}$, the coefficient of friction has an almost constant value, this means that the limiting shear stress has been reached and the shear stresses no longer increases with increasing shear strain rate or sliding velocity. At the high end of the velocity range, above $0.3 \mathrm{~m} / \mathrm{s}$, the friction model predicts a sharp drop. This is not a result of the properties of the surface layer, but a result of the drop of the size of the contact area during sliding, as discussed in Chapter 4.

The influence of the parameters presented in Table 6.1 on the friction behaviour of a tribo-system with this surface layer is studied by varying each of them and comparing the results with the defined model case shown in Fig. 6.1. This is explained in the further sections.

\subsubsection{Influence of the viscosity, $\eta_{L}$}

To study the effect of the viscosity, $\eta_{L}$, in the friction model, the friction at different sliding velocities was calculated using different values of $\eta_{L}$. The results are presented in Fig. 6.2, where it can be seen that at the lower viscosity of the tribo-generated surface layer, $\eta_{L}=10 \mathrm{~Pa}$ s, the coefficient of friction is very low and slowly increases with increasing sliding velocity. Due to the reduction in contact area at high sliding velocities, above $0.3 \mathrm{~m} / \mathrm{s}$, the coefficient of friction decreases. For higher viscosities of the surface layer, the coefficient of friction is higher and increases more rapidly with sliding velocity up to approximately $\mu=1.8$. For high viscosities, such as $\eta_{L}=10^{4} \mathrm{~Pa} \mathrm{~s}$, the coefficient of friction shows a small variation with sliding velocity. For high velocities the friction coefficient found at viscosities higher than $580 \mathrm{~Pa} \mathrm{~s}$ is rather similar. Therefore it can be concluded that the viscosity of the surface layer does not influence the maximum value of the coefficient of friction curve, but it has a strong influence on the dependency between the friction coefficient and the sliding velocity.

Because the dimensionless parameter $\xi$ is proportional to the viscosity of the layer, as seen in Fig. 5.12, higher values of the viscosity translate to higher values of $\xi$ and therefore to higher shear stresses. When the shear stresses in the surface layer reach the limiting shear stress, the surface layer shears plastically. The relative constant value of the coefficient of friction with sliding velocity observed for high viscosity $\left(\eta_{L}=10^{4} \mathrm{~Pa} \mathrm{~s}\right)$, in Fig. 6.2, indicates that the surface layer is sheared plastically. At low viscosity and low sliding velocities the tribo-generated surface layer will shear mainly elastically. 


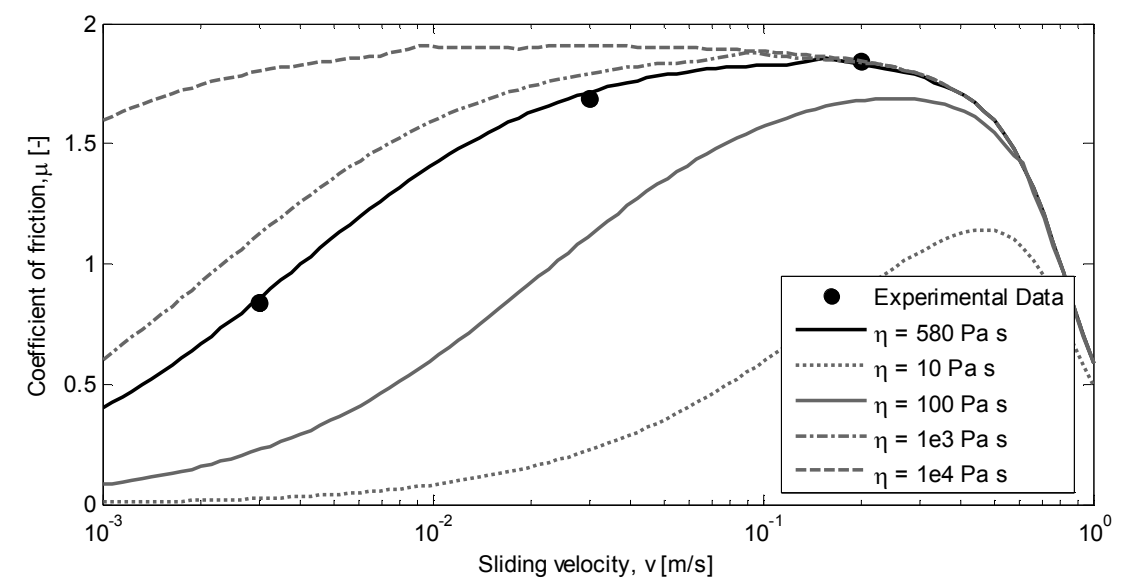

Fig. 6.2: Coefficient of friction as a function of the sliding velctiy at various values of the viscosity parameter of the tribo-generated surface layer $\eta_{L}$.

\subsubsection{Influence of the elastic shear modulus, $G_{L}$}

Fig. 6.3 shows the influence of the elastic shear modulus $G_{L}$ of the surface layer on the coefficient of friction at different sliding velocities. It can be seen that at very low values of the elastic shear modulus, the coefficient of friction is very low and rather constant, and at increasing $G_{L}$ the friction behaviour with respect to the sliding velocity approaches the behaviour found with $G_{L}=0.59 \mathrm{MPa}$. Higher values of $G_{L}$ are not included because the coefficient of friction does not change further.

The elastic shear modulus is inversely proportional to the Deborah number, which was defined in Chapter 5 and the sliding velocity is directly proportional to the Deborah number. Low values of Deborah number means that the increase of shear stresses with shear strain rate behaves more elastic than viscous. At a low shear modulus of the layer, $G_{L}=0.1 \mathrm{kPa}$, the Deborah number is high and the surface layer behaves more viscous, resulting in a low dependency of friction with strain shear rate or with sliding velocity.

A high shear modulus of the layer, $G_{L}$, the Deborah number is low, $D \ll 1$, meaning that the shear stresses increase almost linearly with the shear strain rate, therefore increasing the coeffiecient of friction. However, due to the limiting shear stress, the shear stress in the contact can not increase more than $\tau_{L}$ thus limiting the coefficient of friction. And because the shear stress increases rather elastically with shear strain rate, there is a dependency between the coefficient of friction and the sliding velocity. 


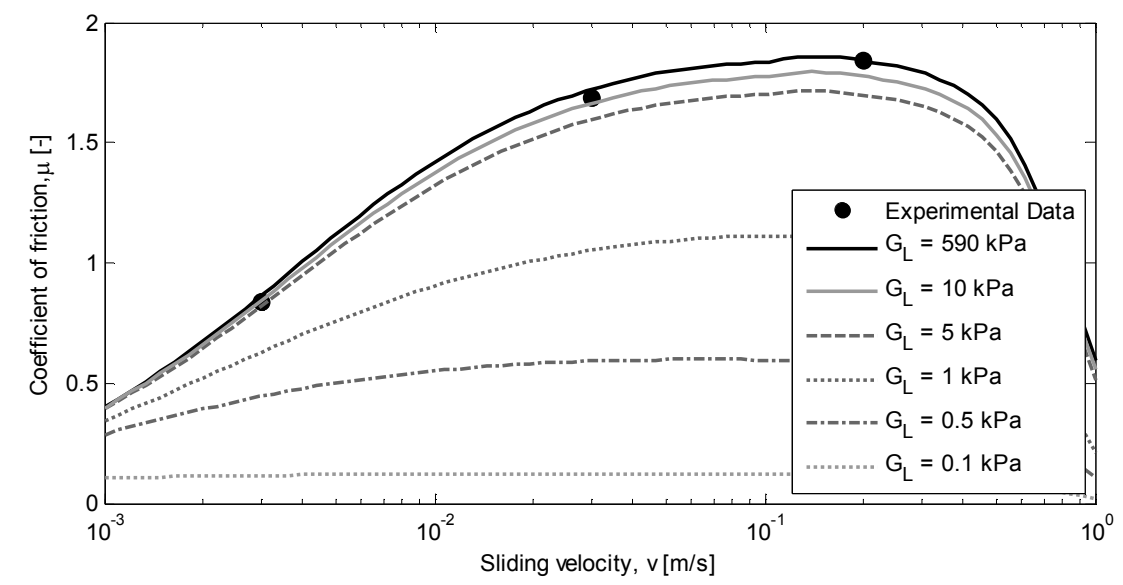

Fig. 6.3: Coefficient of friction as a function of the sliding velocity, for various values of the elastic shear modulus $G_{L}$.

\subsubsection{Influence of the limiting shear stress, $\tau_{\mathrm{L}}$}

The influence of the limiting shear stress on the friction behaviour at different sliding velocities is shown in Fig. 6.4.

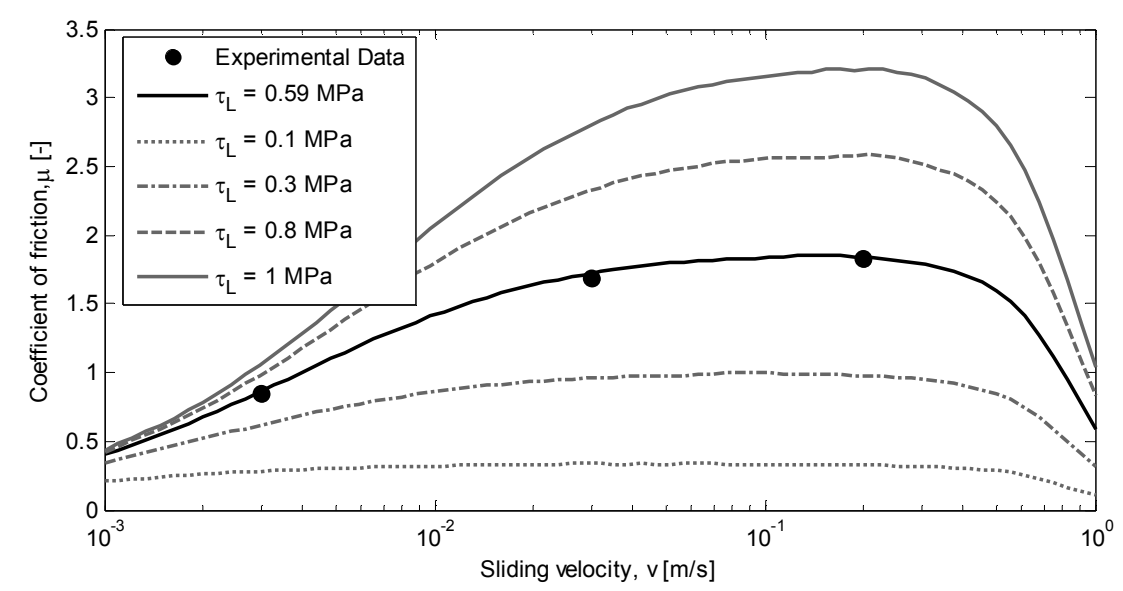

Fig. 6.4: Friction coefficient as a function of the sliding velocity, calculated with various values of the limiting shear stress $\tau_{L}$.

It can be seen that the limiting shear stress is responsible for the level of friction. At the lowest value of the limiting shear stress, the variation of coefficient of friction with sliding velocity is no longer observed, because the 
Friction model

shear stresses in the surface layer are too limited, $\tau / \tau_{\mathrm{L}}=1$, and the surface layer shears plastically at nearly all sliding velocities.

\subsubsection{Influence of the layer thickness, $h$}

The friction coefficient at different sliding speeds is calculated using different surface layer thicknesses; the results are shown in Fig. 6.5. It can be seen that for a very thin surface layer the coefficient of friction is high and rather constant, even at low sliding velocities. For very thick surface layers the coefficient of friction is highly reduced, at least at low sliding velocities. The sliding velocity and the thickness of the surface layer are related to the strain shear rate as $\dot{\gamma}=\mathrm{v} / h$, thus, by modifying the thickness of the surface layer, the strain shear rate is being modified. The thicker the surface layer, the lower the strain shear rate and the lower the stresses in the surface resulting in a low coefficient of friction. The effect of the thickness of the surface layer on the friction behaviour with sliding velocity is inverse to the effect of the viscosity of the surface layer.

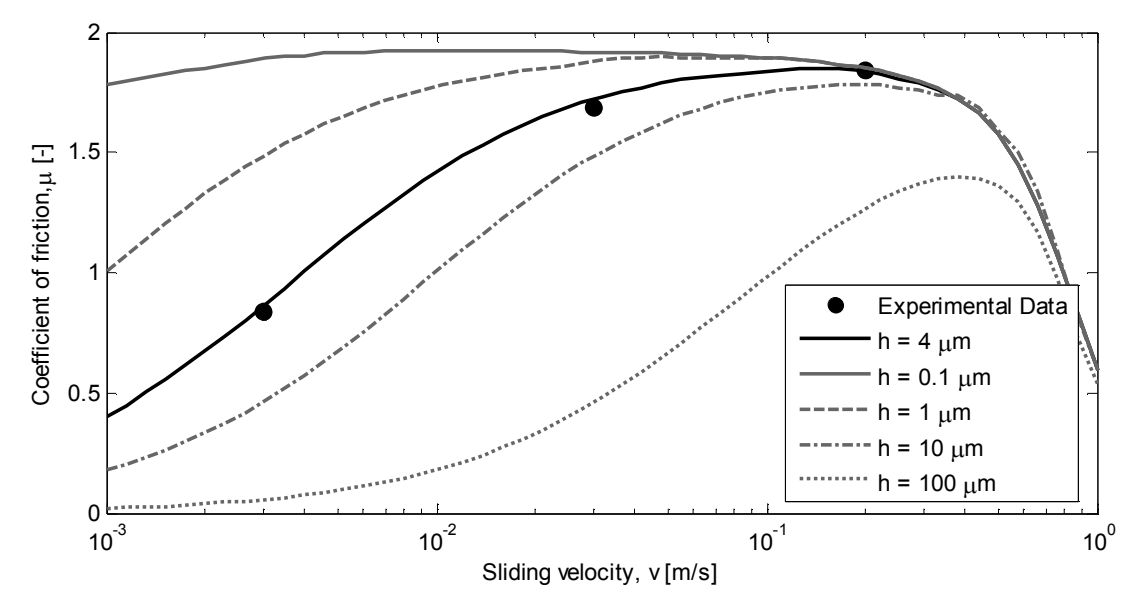

Fig. 6.5: Coefficient of friction as a function of the sliding velocity at different thickness of the surface layer $h$.

\subsection{Friction behaviour of fibre reinforced EPDM}

In the present thesis the friction behaviour of a tribo-system composed of a rigid spherical indenter in sliding contact with a fibre reinforced elastomer is studied. The addition of fibres into the elastomer in a preferred orientation causes anisotropy to the composite material. From Chapter 4 it is known that the contact area is influenced by the mechanical properties of the contacting elastomers. A fibre reinforced elastomer will have improved 
mechanical properties compared to the unreinforced elastomer, and therefore the size of the contact area under similar loading conditions will be smaller. Considering the different contact areas generated at different degrees of reinforcement and the shear stresses calculated for the unreinforced EPDM, the friction coefficient in fiber reinforced elastomer is calculated. In the present section, the influence of the orientation of fibres in friction experiments are shown, followed by the effect of fibres on the friction behaviour of tribo-system.

Friction experiments were performed using various fibre reinforced EPDM samples, with with $5 \mathrm{phr}$ of Twaron $\AA$ fibres oriented randomly $(R)$ and in longitudinal $(L)$ direction. These friction experiments were performed at a sliding distance of $500 \mathrm{~m}$, a constant sliding velocity of $0.2 \mathrm{~m} / \mathrm{s}$ and a load of $2 \mathrm{~N}$, on samples with a pre-generated surface layer. The results of these friction experiments, together with the friction experiments on the unreinforced EPDM are shown in Fig. 6.6.

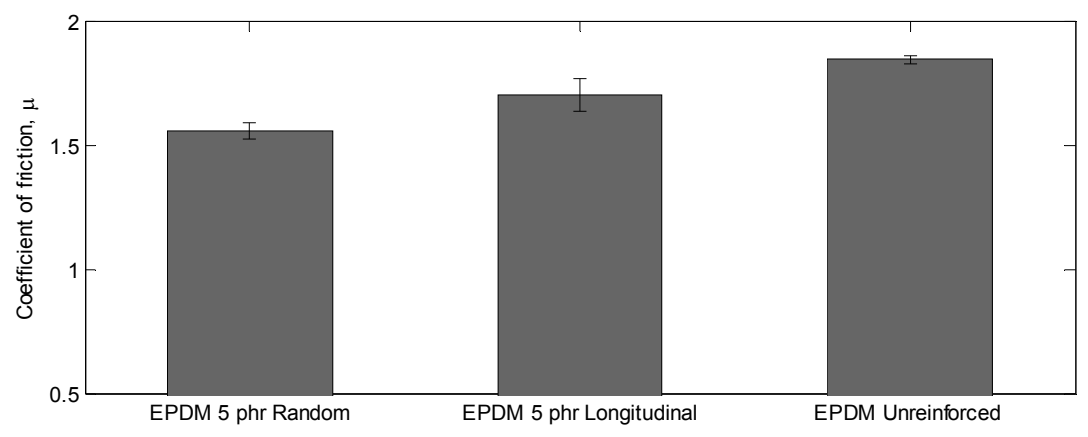

Fig. 6.6: Coefficient of friction at different orientations of reinforcement related to the sliding direction.

It can be seen that the orientation of fibres does influence the frictional behaviour of the tribo-system. In the case of unreinforced EPDM, the coefficient of friction is $\mu=1.84 \pm 0.015$. The reinforced samples have fibres oriented in two configurations. Longitudinal orientation, which means that the fibres are oriented along the sliding direction and in which a coefficient of friction of $\mu=1.70 \pm 0.06$ is obtained; and random orientation, which means that the fibres are oriented randomly in the elastomer, giving a coefficient of friction $\mu=1.55 \pm 0.03$. 
Friction model

\subsubsection{Influence of fibres on the friction behaviour}

As shown previously, for unreinforced EPDM, the tribo-generated surface layer is of great importance for the friction behaviour. To study this phenomenon for fibre reinforced materials, friction experiments have been performed at different sliding velocities.

Similar to the experiments for unreinforced EPDM, the tribo-generated surface layer was developed by sliding the rigid spherical indenter $(\mathrm{R}=12.5 \mathrm{~mm})$ at a sliding velocity of $0.2 \mathrm{~m} / \mathrm{s}$ and an applied normal load of $2 \mathrm{~N}$, over a distance of $500 \mathrm{~m}$. The coefficient was measured at an applied normal load of $F_{N}=2 \mathrm{~N}$ using a spherical rigid indenter with a radius of $R=12.5 \mathrm{~mm}$.

The comparison between the coefficient of friction of the unreinforced and reinforced EPDM is shown in Fig. 6.7. It can be seen that in both reinforced and unreinforced elastomer in the presence of surface layer, there is a drop in the coefficient of friction at low sliding velocities. This means that in the fibre reinforced elastomer, the friction is also highly influenced by the tribogenerated surface layer.

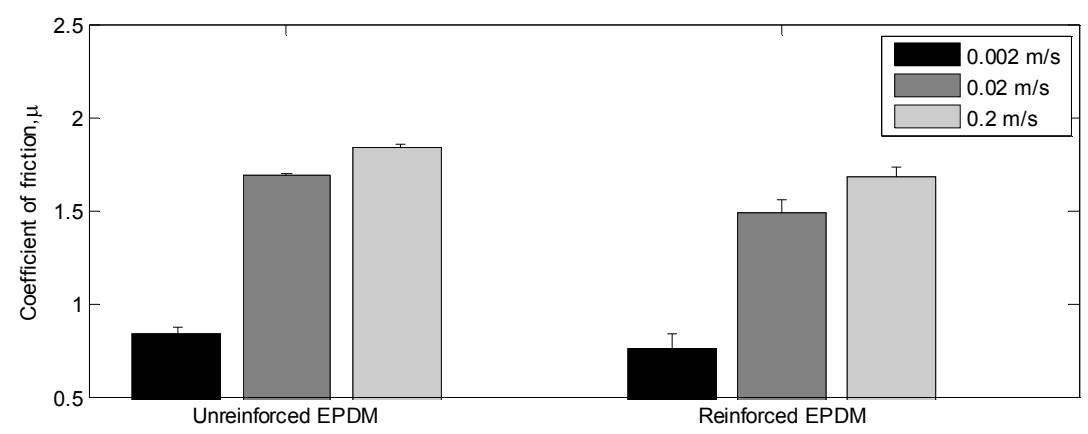

Fig. 6.7: Coefficient of friction in EPDM and EPDM 5 phr, with fibres oriented longitudinally to the sliding direction.

To model the friction behaviour of the anisotropic viscoelastic surface, the size of the contact area and the shear stresses on the contact are required. The size of the contact area can be calculated using the creep compliance functions obtained for the fibre reinforced EPDM, such as shown in Fig. 4.20. The creep-complaince parameters for EPDM with longitudinal and randomly oriented fibres are summarized in Table 6.2. The shear stresses in the surface were calculated using the parameters of the surface layer for unreinforced EPDM. Both the experimental friction results and the calculated friction results are shown in Fig. 6.8. 
Chapter 6

Table 6.2: Parameters of creep compliance function for EPDM and reinforced EPDM, with fibres aligned in the sliding direction and randomly.

\begin{tabular}{ccccccc}
\hline \multicolumn{3}{c}{ Unreinforced EPDM } & \multicolumn{2}{c}{ EPDM 5 phr Longitudinal } & \multicolumn{2}{c}{ EPDM 5phr Random } \\
\hline$i$ & $\phi_{i}\left(\mathrm{MPa}^{-1}\right)$ & $\lambda_{i}(\mathrm{~s})$ & $\phi_{i}\left(\mathrm{MPa}^{-1}\right)$ & $\lambda_{i}(\mathrm{~s})$ & $\phi_{i}\left(\mathrm{MPa}^{-1}\right)$ & $\lambda_{i}(\mathrm{~s})$ \\
& & & & & & \\
$\phi_{r}$ & $3.49 \mathrm{E}-01$ & & $2.90 \mathrm{E}-01$ & & $2.81 \mathrm{E}-01$ & \\
1 & $4.69 \mathrm{E}-02$ & 0.0064 & $3.82 \mathrm{E}-02$ & 0.0066 & $1.97 \mathrm{E}-03$ & 0.0081 \\
2 & $4.90 \mathrm{E}-02$ & 0.0713 & $4.05 \mathrm{E}-02$ & 0.0711 & $1.07 \mathrm{E}-02$ & 0.0523 \\
3 & $8.23 \mathrm{E}-02$ & 0.7284 & $6.79 \mathrm{E}-02$ & 0.7431 & $3.12 \mathrm{E}-02$ & 0.4991 \\
\hline
\end{tabular}

It can be seen from Fig. 6.8 that the friction model agrees well with the experimental results for both the reinforced and unreinforced EPDM, as well as for the different orientation of fibres. The reduction in coefficient of friction in the case of fibre reinforced elastomer is due to a reduction of the size of the contact area as a result of the reinforcement.

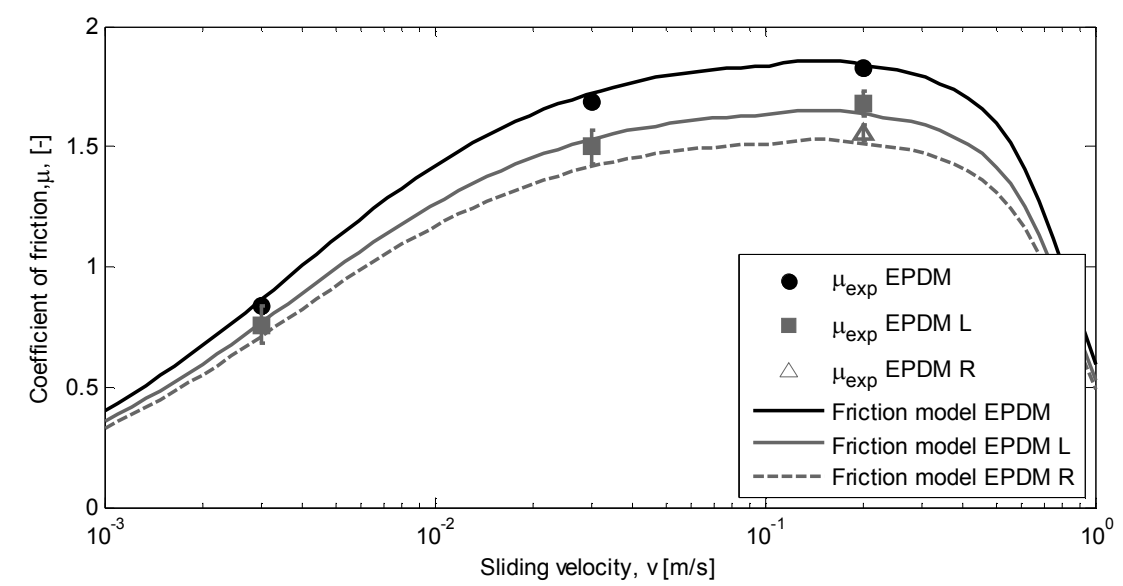

Fig. 6.8: Coefficient of friction depending on the sliding velocity for unreinforced and reinforced EPDM with fibres oriented longitudinally $(L)$ and randomly $(R)$ to the sliding direction.

From Fig. 6.6 it can be seen that the lowest coefficient of friction was obtained for EPDM with randomly oriented fibres. The largest increase in material properties generally occurs in the case of one preferred orientation, especially when the orientation is in the direction of indentation. Randomly oriented fibres can achieve an increase in the material properties 
Friction model

due to reinforcement in both tension and shear, as discussed in Chapter 4 . This is illustrated in Fig. 6.6 where for randomly oriented fibres the lowest coefficient of friction is obtained.

\subsection{Summary}

In the present thesis, the tribosystem composed of a fibre reinforced elastomer in sliding contact with a rigid spherical indenter is studied. The sliding velocity used in the model tribo-system is $0.2 \mathrm{~m} / \mathrm{s}$ and the applied normal load used is $2 \mathrm{~N}$. From preliminary experiments on dry and boundary lubricated elastomer surfaces it was concluded that the adhesional mechanism of friction is the most influential in the present tribosystem. To be able to predict the friction due to the adhesion mechanism of friction, the size of the contact area and the shear stresses inside the contact area need to be known.

In the present chapter the size of the contact area, modelled in Chapter 4, and the shear model, developed in Chapter 5 , are combined to describe the friction of the reference system as a function of the sliding velocity. The results of this model agree with friction experiments performed on samples with a pre-formed tribo-generated surface layer for both unreinforced and reinforced EPDM, with fibres oriented randomly and along the sliding direction $(\mathrm{L})$. Therefore the reduction of the coefficient of friction is a result of the reduction in the size of the contact area. Furthermore, the properties of the surface layer are not affected by the presence of the fibres for reinforcing the elastomer.

A low value of the contact area and therefore the coefficient of friction can be obtained by increasing the tensile stiffness $E$ and the shear stiffness $G$. Adding fibres in longitudinal direction increases only the tensile properties, whilst a random orientation of fibres also increases the shear properties. This means that a low friction can also be achieved by adding randomly oriented fibres. 



\section{Chapter 7 Conclusions and recommendations}

In this thesis the tribological behaviour, i.e. contact and friction, of short fibre reinforced elastomers in contact with a rigid surface was studied. The present chapter closes this research. A short overview of the studied tribological system and of the developed contact and friction models is given, followed by a summary of the most relevant conclusions presented in each chapter. Finally, some recommendations for future research are given.

\subsection{Overview of this thesis}

The increase in material properties of a fibre reinforced composite depends on the type of fibre used, the concentration and the dimension (length) of the fibres as well as the effective orientation of the fibres in the matrix. In the present thesis these parameters are encapsulated in terms of the reinforcement, which is the increase in material properties in both tension and shear. The elastomer composite is considered to behave as a continuum viscoelastic anisotropic material.

A model tribo-system was defined based on the tyre-road contact. For this model system, it was shown that the most influential friction mechanism is the adhesion mechanism. Therefore the contact area and the shear stresses during sliding are studied.

Two static contact models that consider the combined anisotropy and viscoelasticity of the material were developed. As the present tribo-system considers sliding contact, the contact model was extended to include the effect of the sliding velocity on the contact size.

From observations of the surfaces of elastomeric materials after tribological experiments it was concluded that a tribo-generated surface layer develops on the elastomeric surface. The friction behaviour of this surface layer was modelled using a shear model, considering viscoelastic behaviour of the surface layer. The results of the model for reinforced and unreinforced EPDM show good agreement with experiments, validating the friction model.

\subsection{General conclusions}

The general conclusions of the present thesis are fourfold:

- The static contact between a fibre reinforced elastomer and a rigid indenter was described by a contact model, showing that the 
contact area strongly depends on the material properties in both tensile and shear governed by the fibre reinforcement.

- An experimental investigation showed that the contact area during sliding remains constant up to a certain sliding velocity, after which it drops sharply.

- The friction behaviour of a fibre reinforced elastomer in sliding contact against a rigid spherical indenter was described by an adhesion-based friction model. The results of the model are in agreement with the experimental results. It is concluded that the friction behaviour is strongly affected by a tribo-generated surface layer. Additionally it was shown that, for the present tribo-system, the surface properties of this layer hardly change with the presence of fibres for reinforcing the elastomer.

- A lower friction is obtained with composites in which the contact area is reduced. For a fibre reinforced elastomer a smaller contact area can be obtained by aligning the fibres in the direction of the indentation (highest tensile reinforcement) or when the fibres are randomly oriented (combination of high tensile reinforcement and high shear reinforcement).

\subsubsection{Conclusions Chapter 4}

The main difference between the two developed contact models is the range of anisotropy in which they are accurate and the input data that they require. The developed orthotropic viscoelastic isochronous contact model allowed a parameter study of the material properties in different directions of the elastomer. It was found that improving the material properties in shear as well as in tension can cause a significant reduction in the size of the contact area. A qualitative study of the contact behaviour of an anisotropic material was performed with this contact model. Considering that the shear modulus of the composite is hardly influenced by the fibres, the smallest contact area is obtained in the composite with fibres oriented along the indentation direction rather than with fibres that are oriented in the plane of indentation, and the biggest contact area is found with an unreinforced elastomer. Elastomers reinforced with randomly oriented fibres can present a lower contact area than other orientations because the reinforcement occurs in both tension and shear. 
Due to the relatively low reinforcement of the studied composite, differences of $20 \%$ in the creep compliance between reinforced and unreinforced EPDM, and the reduced number of input parameters, the unidirectional viscoelastic contact model was used to describe the static contact behaviour of the fibre reinforced elastomer. From the static contact models it is concluded that the contact area reduces with increasing material properties, both in tensile and shear.

During sliding contact, the size of the contact area reduces, depending on the sliding velocity. This was experimentally investigated and an arctan function was proposed to describe the contact area as a function of the sliding velocity.

\subsubsection{Conclusions Chapter 5}

Due to sliding friction, the properties of the surface of the elastomer change. This layer, or tribo-generated surface layer, was found to have poor mechanical properties and to have a large influence on the frictional behaviour of elastomers in sliding contact with a rigid indenter. Therefore this layer is taken into account in order to model the friction behaviour in the present tribo-system.

The shear stresses occurring in this layer are modelled following existing shear models of interfacial layers, considering that the interfacial layer behaves viscoelastically. The parameters defining the behaviour of the surface layer were determined in relation with measurement results of indentation and friction experiments. After this, the model of Elsharkawy \& Hamrock [69] was found to be the most suitable for describing the shear behaviour of the tribo-generated surface layer.

\subsubsection{Conclusions Chapter 6}

The friction in the present tribo-system is calculated using the size of the contact area calculated with the contact model developed in Chapter 4, and the shear stresses calculated with the model presented in Chapter 5. With this, the coefficient of friction in the presence of a pre-formed surface layer is calculated as a function of the sliding velocity.

The results of the friction model and friction experiments for reinforced and unreinforced EPDM are very much in agreement and therefore the conclusion is that the reduction in the coefficient of friction at different reinforcement is due to a reduction in the size of the contact area and that the presence of fibres hardly affect the properties of the surface layer. 


\subsection{Discussion and recommendations}

Based on the present research, some recommendations regarding future research are discussed, followed by a summary of these recommendations.

\subsubsection{Influence of anisotropy on the viscoelastic contact}

The viscoelastic orthotropic isochronous contact model is difficult to implement due to the large amount of input parameters needed. However, this model is particularly suited to describing the contact behaviour of highly anisotropically-reinforced materials, for example long (continuous) fibre reinforced polymers.

To characterize the level of reinforcement of the elastomer composite, it is recommended to measure the material properties in shear besides those in tension. In the elastic anisotropic case the isotropic relation $G=E /(2(1+v))$ does not hold and in the viscoelastic case the contact area should be calculated with a creep compliance function measured in shear mode.

\subsubsection{Influence of the tribo-generated surface layer on friction.}

Chapter 3 shows the coefficients of friction obtained from sliding friction experiments performed at different sliding velocities, over a sliding distance of $500 \mathrm{~m}$. At a sliding velocity of $v=0.2 \mathrm{~m} / \mathrm{s}$ a tribo-generated surface layer was found; this layer was analyzed (properties and frictional behaviour) in Chapter 5 and Chapter 6.

Fig. 7.1 shows SEM images of the tribo-generated surface layers obtained from sliding experiments at a load of $2 \mathrm{~N}$ and sliding velocities of 0.2 and $0.4 \mathrm{~m} / \mathrm{s}$. It can be seen that for a sliding velocity of $0.2 \mathrm{~m} / \mathrm{s}$ the thickness of the developed layer is $4 \mu \mathrm{m}$, whilst for $0.4 \mathrm{~m} / \mathrm{s}$ the layer has a thickness of $8.7 \mu \mathrm{m}$, which is a possible indication that the tribo-generated surface layer gradually develops and its properties depend on the operational conditions. 
Conclusions and recommendations
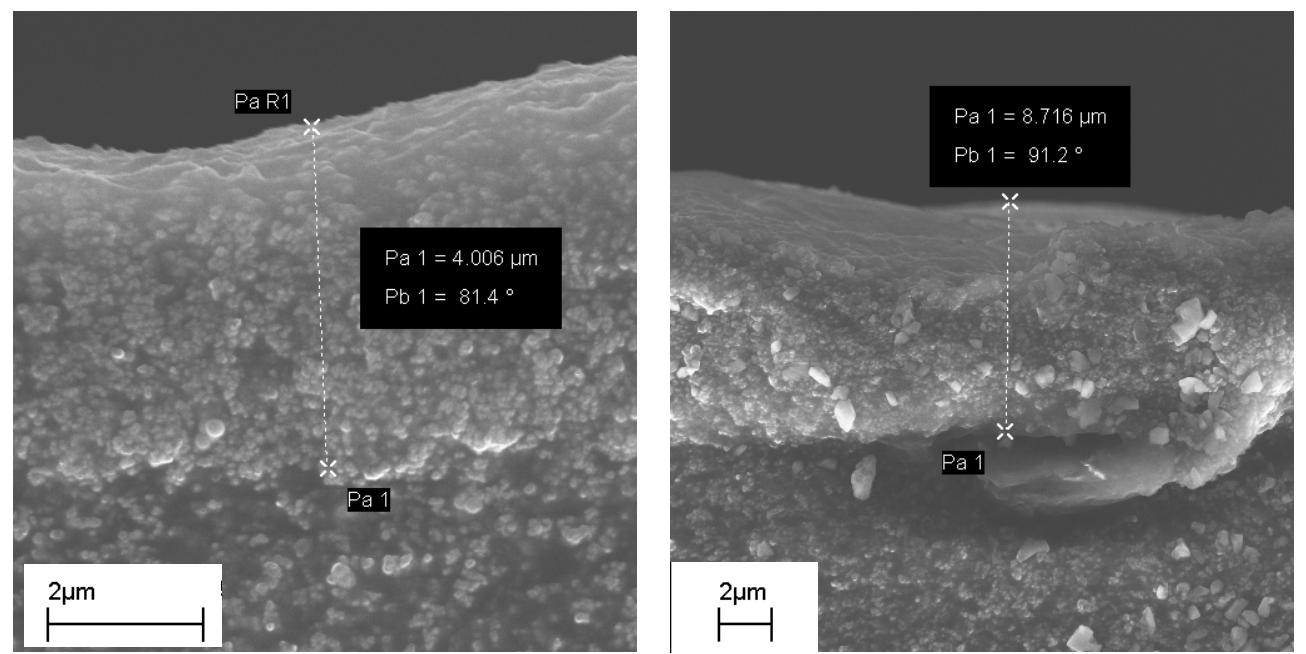

Fig. 7.1: SEM images of the tribo-generated surface layers created at different sliding velocities: at $0.2 \mathrm{~m} / \mathrm{s}$ on the left and $0.4 \mathrm{~m} / \mathrm{s}$ on the right, with a thickness of $4 \mu \mathrm{m}$ and $8 \mu \mathrm{m}$ respectively.

To estimate the influence of the development of the surface layer on friction, the model has been modified to include a linear increase of the thickness of the surface layer as a function of sliding velocity. Friction experiments perfomed at different sliding velocities with unreinforced EPDM are shown in Fig. 7.2 a) and those with reinforced EPDM with fibres oriented longitudinally to the sliding direction are shown in Fig. 7.2 b). The grey circles represent the coefficient of friction obtained during sliding for samples with a fully developed pre-formed surface layer, as shown in Chapter 6. The black squares are the results of friction experiments performed on samples without a pre-formed surface layer. At the start of these friction experiments there is no pre-formed surface layer. After sliding over a distance of $500 \mathrm{~m}$ a wear track is visible and then the friction is measured. It can be seen that in the absence of a pre-formed surface layer, the friction model shows a better prediction of the coefficient of friction when the thickness depends linearly on the sliding velocity than when a constant thickness is assumed. 
a)

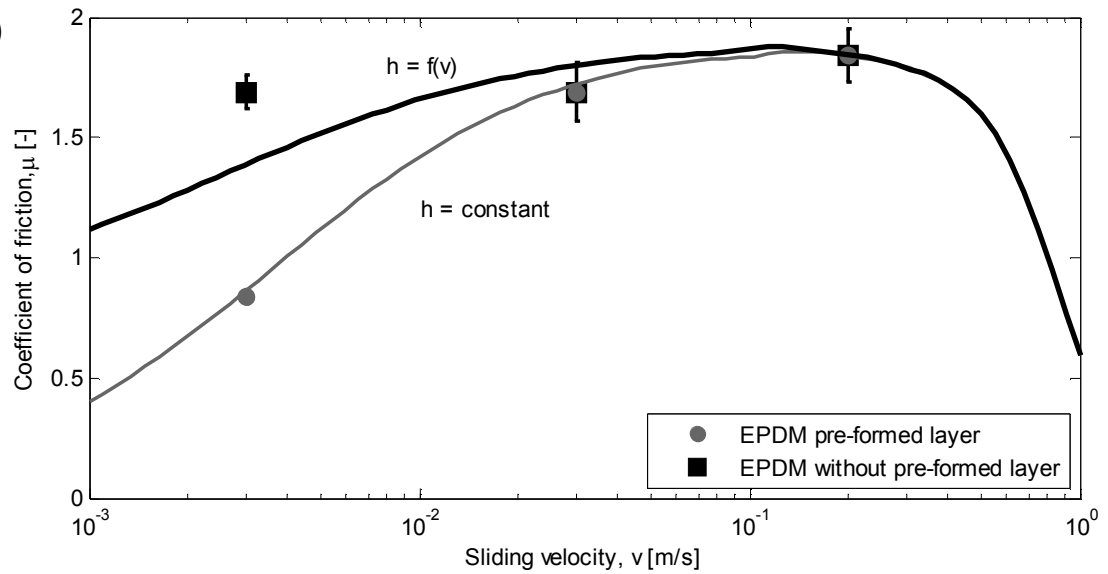

b)

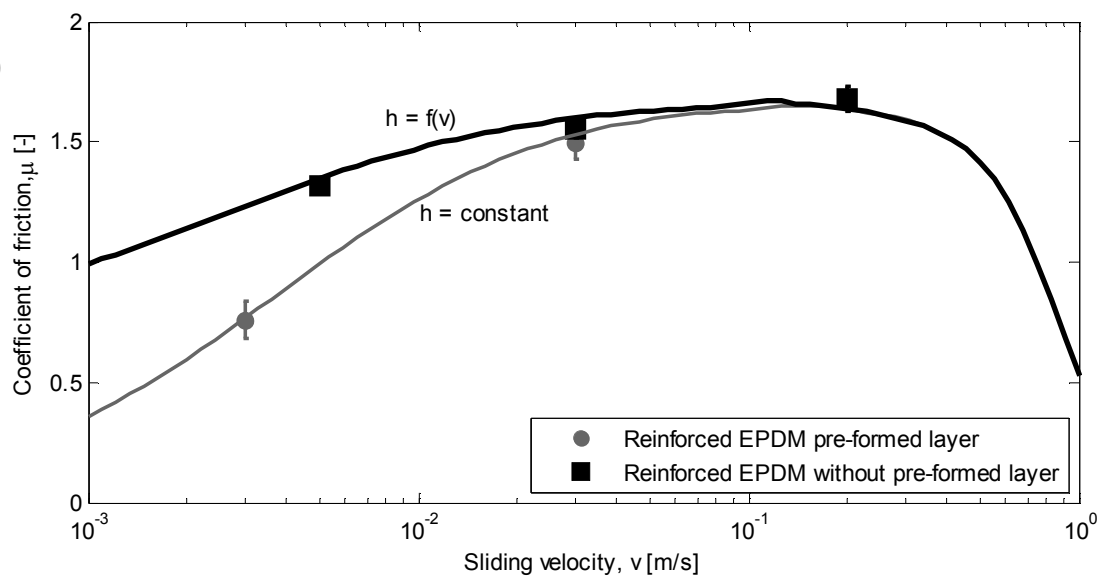

Fig. 7.2: Coefficient of friction as a function of sliding velocity, with linearly increasing thickness of the surface layer, for unreinforced EPDM on a) and for reinforced EPDM on b).

Further research concerning the formation of the tribo-generated surface layer as a function of operational conditions, as well as the fibre content of the elastomer, is recommended. Furthermore, because the surface layer has poor material properties compared to the elastomer bulk and the elastomer bulk has such low stiffness compared to other kinds of materials (plastics, metals, ceramic), measuring the viscoelastic properties of the surface layer is very challenging. Further research is recommended into the measurement of the material properties of tribo-generated surface layers in elastomer systems. 


\subsubsection{Influence of sliding on the size of the contact area}

To consider the effect of sliding velocity on the size of the contact area, experiments were performed and an arctan function was proposed to describe the change in size of the contact area as a function of sliding velocity. It is expected that the transition velocity at which the contact area starts decreasing depends on the material properties, such as the moduli in shear and tension. This shift of the transition velocity is shown schematically in Fig. 7.3.

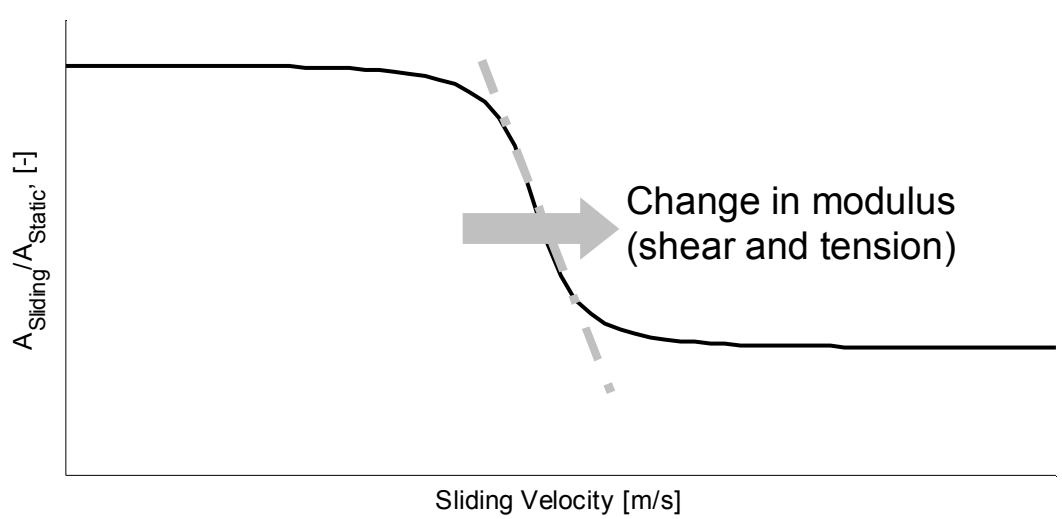

Fig. 7.3: Diagram of the variation of the size of contact area with sliding velocity, and dependency of the transition velocity on moduli in shear and tension.

Therefore further research concerning the variation of size of the contact area as a function of operational conditions and material properties is recommended.

\subsubsection{Influence of deformation on friction}

It has been shown that the main friction mechanism for reinforced elastomers in sliding contact is adhesion. However, the deformation mechanism of friction could become relevant for different operational conditions. Differences in the tribological system can be caused by, for instance, a different pressure distribution (geometry of the counter surface) and temperature, resulting in different material behaviour. Therefore to complete the analysis in friction, the deformation mechanism of friction should be incorporated into the present friction model, making it suitable for different types of tribo-systems, for instance polymers in general. 


\subsubsection{Summarized recommendations}

- Research concerning the formation of the tribo-generated surface layer, especially the variation of thickness with operational conditions (pressure, temperature and velocity), is recommended.

- Further research is recommended into the measurement of the material properties of tribo-generated surface layers on elastomers.

- Research relating the variation of the size of the contact area to the sliding velocity and the material properties is recommended.

- To complete the analysis in friction, the deformation mechanism of friction should be incorporated into the present friction model. 


\section{Appendices}

Appendix A Tyre - road contact tribo-system.................................... 127

Appendix B Hertz theory ................................................................. 129

Appendix C Characterization of EPDM …..................................... 131

Appendix D Pin-on-disk experiments setup.......................................... 133

Appendix E Contact angle measurements ....................................... 135

Appendix $F$ Determining transient function from dynamic tests ..............137

Appendix G Full anisotropic contact model ....................................... 139 



\section{Appendix A Tyre - road contact tribo-system}

In the contact patch of the asphalt with the tyre tread, the stones of the asphalt are sliding against a piece of rubber from the tread. The deformation of the tyre in the contact causes a difference in relative velocity between a point of the tread of the tyre that is in contact with the asphalt, see Fig. A.

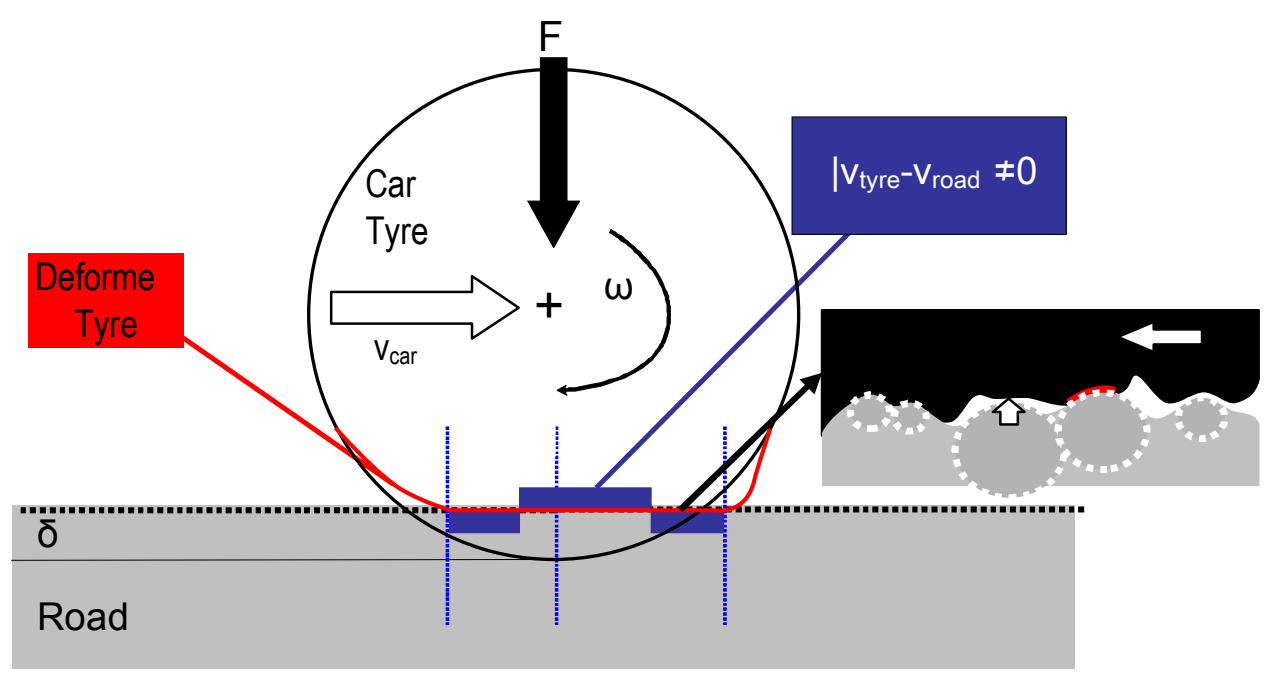

Fig. A: Schematic representation of the tyre-road contact.

The tyre-road contact is shown schematically in Fig. A. At the right hand side, the local contact is shown: the rubber (black) is sliding at that location over the road (grey). The blue areas in the contact patch indicate the sign of relative velocity between a point on the car tyre tread and the road. Typical sliding velocity between the tyre and the road at a cruising speed of $80 \mathrm{~km} / \mathrm{h}$ is around $0.2 \mathrm{~m} / \mathrm{s}$ and the average contact pressure between the tyre and road is around $0.25 \mathrm{MPa}$. 



\section{Appendix B Hertz theory}

For the general Hertzian problem of ellipsoid solids in contact, the required inputs are: $R_{1 x}, R_{1 y}, R_{2 x}, R_{2 y}$, the normal load $F_{N}$ and the equivalent elastic modulus, E*.

The semi-axes of the contact ellipse are:

$$
\begin{aligned}
& a_{x}=a_{x}^{*}\left(\frac{3 F_{N} R}{E_{*}}\right)^{\frac{1}{3}} \\
& a_{y}=a_{y}^{*}\left(\frac{3 F_{N} R}{E_{*}}\right)^{\frac{1}{3}}
\end{aligned}
$$

Normal indentation:

$$
\delta=\delta^{*}\left(\frac{9 F_{N}{ }^{2}}{8 R\left(E_{*}\right)^{2}}\right)^{\frac{1}{3}}
$$

Mean contact pressure:

$$
p_{m}=\frac{F_{N}}{\pi a_{x} a_{y}}
$$

Maximum Hertizan pressure:

$$
p_{0}=\frac{3}{2} p_{m}
$$

where

$$
\begin{gathered}
\frac{1}{R}=\frac{1}{R_{1 x}}+\frac{1}{R_{1 y}}+\frac{1}{R_{2 x}}+\frac{1}{R_{2 y}} \\
\frac{1}{E_{*}}=\frac{1-v_{1}^{2}}{E_{1}}+\frac{1-v_{2}^{2}}{E_{2}}
\end{gathered}
$$




$$
\begin{gathered}
a_{x}^{*} \approx \kappa^{\frac{1}{3}}\left[\frac{2}{\pi} E(m)\right]^{\frac{1}{3}} \\
a_{y}^{*} \approx \kappa^{\frac{-2}{3}}\left[\frac{2}{\pi} E(m)\right]^{\frac{1}{3}} \\
\delta^{*} \approx \kappa^{\frac{2}{3}}\left[\frac{2}{\pi} E(m)\right]^{\frac{-1}{3}}\left[\frac{2}{\pi} K(m)\right]
\end{gathered}
$$

The elliptical integrals are:

$$
\begin{gathered}
E(m) \approx \frac{\pi}{2}(1-m)\left[1+\frac{2 m}{\pi(1-m)}-\frac{1}{8} \ln (1-m)\right] \\
K(m) \approx \frac{\pi}{2}(1-m)\left[1+\frac{2 m}{\pi(1-m)} \ln \left(\frac{4}{\sqrt{1-m}}\right)-\frac{3}{8} \ln (1-m)\right]
\end{gathered}
$$

and

$$
\begin{gathered}
\kappa \approx\left[1+\sqrt{\frac{\ln (16 / \vartheta)}{2 \vartheta}}-\sqrt{\ln (4)}+0.16 \ln (\vartheta)\right]^{-1} \\
m=\sqrt{1-\kappa^{2}} \text { and } \kappa=\frac{a}{b} \text { for } a<b \\
\vartheta=\frac{R_{x}}{R_{y}} \quad \text { for } \quad 0<\vartheta \leq 1
\end{gathered}
$$

where the principal combined radii of curvature, $R_{x}$ and $R_{y}$, are given by:

$$
\frac{1}{R_{x}}=\frac{1}{R_{1 x}}+\frac{1}{R_{2 x}} \quad \text { and } \quad \frac{1}{R_{y}}=\frac{1}{R_{1 y}}+\frac{1}{R_{2 y}}
$$




\section{Appendix C Characterization of EPDM}

Ethylene Propylene Diene Rubber, EPDM (Keltan® 8340A with 5.5 weight percent ENB content and respectively 55 and 39.5 weight percents of ethylene and propylene contents, from DSM Elastomers, the Netherlands.)

Table C: Recipe of EPDM

\begin{tabular}{cc}
\hline & phr \\
\hline Keltan 8340A & 100 \\
Stearic Acid & 1 \\
FEF N-550 & 105 \\
Sunpar 2280 & 60 \\
TMQ & 1.25 \\
PEG 2000 & 2.5 \\
Perkadox 14/40 & 7.5 \\
TRIM 50 & 4 \\
\hline
\end{tabular}

Mechanical properties were determined according to DIN 53504 with 5 S2 type tensile bars, a cross-head speed of $200 \mathrm{~mm} / \mathrm{min}$ using a $1 \mathrm{kN}$ load cell installed at a tensile testing machine from Zwick $\mathrm{GmbH}$, Ulm, Germany. The elastic modulus was obtained between $1-2 \%$ of strain and an average value of $4.39 \pm 0.14 \mathrm{MPa}$ was determined.

To determine the time dependent properties, DMA measurements using a frequency sweep at $0.5 \%$ strain without pre-strain were performed in shear mode with the same EPDM material. 



\section{Appendix D Pin-on-disk experiments setup}

The experiments were conducted in a pin-on-disk tribo-tester (CSEM), placed in a climate chamber to control temperature and humidity (HC 4057, Heraus, Vötsch). The tested specimens are a steel ball (the pin) pressed against the rotating elastomer specimen (the disk).

The temperature was set at $23^{\circ} \mathrm{C}$ and the humidity was set at $60 \%$. The indenter and elastomer disk remained in acclimatized conditions for 36 hours prior to the sliding friction experiments. The pin used is a SKF ball code 10RB-25 mm, shown in Fig. D.
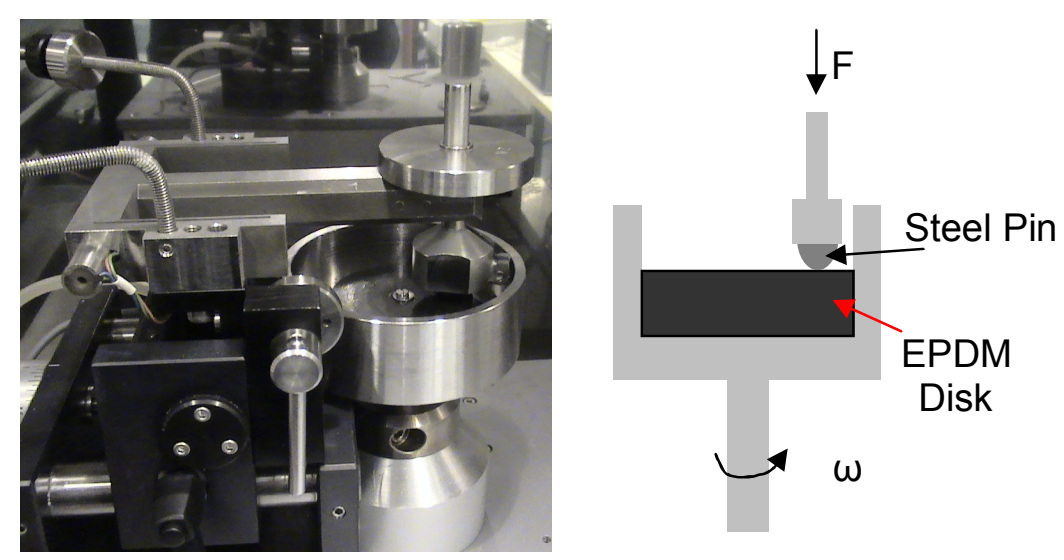

Fig. D: On the left, picture of the pin-on-disk setup, inside the climate chamber. On the right, a schematic representation of the pin-on-disk setup.

The EPDM disks were made by IPF, following the recipe given in Appendix C. They have a diameter of $60 \mathrm{~mm}$ and a thickness of $10 \mathrm{~mm}$. The fibre reinforced elastomer has a concentration of 5 parts per hundred rubber (phr) of Twaron $®$ aramid fibres. The fibres have a length of $3 \mathrm{~mm}$ and a diameter of $12 \mu \mathrm{m}$. 



\section{Appendix E Contact angle measurements}

Adhesion between two surfaces can be quantified by calculating the work of adhesion between the contacting surfaces. The work of adhesion represents the energy that must be applied in order to separate the contacting bodies and is given by Dupre's equation:

$$
W_{12}=\gamma_{1}+\gamma_{2}-\gamma_{12}
$$

where $\gamma_{1}$ and $\gamma_{2}$ indicates the surface free energy or surface tension of the two materials in contact and $\gamma_{12}$ the interfacial tension.

The surface free energy is calculated from the measured contact angles using water $\left(\mathrm{H}_{2} \mathrm{O}\right)$ and diiodomethane $\left(\mathrm{CH}_{2} \mathrm{I}_{2}\right)$ shown in Table $\mathrm{E}$. The contact angles are measured using a Dataphysics OCA 20 contact angle measuring device.

Table E: Results of contact angle measurements and surface tension.

\begin{tabular}{|c|c|c|c|c|c|}
\hline \multirow[t]{2}{*}{ Material } & \multicolumn{2}{|c|}{ Angle (degrees) } & \multicolumn{3}{|c|}{ Surface Tension $\left(\mathrm{mJ} / \mathrm{m}^{2}\right)$} \\
\hline & $\mathrm{H} 2 \mathrm{O}$ & $\mathrm{CH} 2 \mathrm{I} 2$ & Dipolar & Polar & Total \\
\hline $\mathrm{H} 2 \mathrm{O}$ & - & 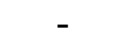 & 21.8 & 51 & 72.8 \\
\hline $\mathrm{CH} 2 \mathrm{I} 2$ & - & - & 50.8 & 0 & 50.8 \\
\hline EPDM & 102.9 & 57 & 31.7 & 2.8 & 34.5 \\
\hline Steel 100Cr6 & 124.2 & 139.8 & 29 & 17.2 & 46.2 \\
\hline EPDM/Steel & - & - & - & - & 10.48 \\
\hline
\end{tabular}

To calculate the surface tension between the elastomer, EPDM, and steel surface the harmonic mean equations from $\mathrm{Wu}$ [72] were used. This method is widely used to calculate the surface tension in polymers and is based on the surface tension between two surfaces being formed by a dispersive (dipolar) and polar component. Using the subscript $L$ for the liquids and $s$ for the solids and the superscript: $p$ and $d$ for the polar and dipolar components [72] the surface energy $\gamma_{s}=\gamma_{s}^{p}+\gamma_{s}^{d}$ can be calculated by solving Eq. E.2 for each surface:

$$
\left(1-\cos \theta_{L}\right) \gamma_{L}=\frac{4 \gamma_{L}^{p} \gamma_{s}^{p}}{\gamma_{L}^{p}+\gamma_{s}^{p}}+\frac{4 \gamma_{L}^{d} \gamma_{s}^{d}}{\gamma_{L}^{d}+\gamma_{s}^{d}}
$$


Appendix E

After doing this for both the EPDM and the steel, the interfacial tension between the two contacting surfaces is obtained by solving Eq. E.3, where the subscripts 1 and 2 indicate the two materials:

$$
\gamma_{12}=\gamma_{1}+\gamma_{2}-\frac{4 \gamma_{1}^{p} \gamma_{2}^{p}}{\gamma_{1}^{p}+\gamma_{2}^{p}}-\frac{4 \gamma_{1}^{d} \gamma_{2}^{d}}{\gamma_{1}^{d}+\gamma_{2}^{d}}
$$

The calculated values for the surface tension and the interfacial tension are listed in Table $\mathrm{E}$. The work of adhesion between the steel surface and the EPDM surface is then given by

$$
W_{12}=34.5+46.2-10.5=70.2 \mathrm{~mJ} / \mathrm{m}^{2}
$$




\section{Appendix $F$ Determining transient function from dynamic tests}

The storage and loss modulus in shear can be fitted according to a Prony series to obtain the equilibrium modulus, $G_{e}$, relaxation strengths, $G_{i}$ and relaxation times, $\tau_{i}$, of the stress relaxation function [47].

$$
\begin{gathered}
G^{\prime}(\omega)=G_{e}+\sum_{i=1}^{n} G_{i} \cdot \frac{\left(\omega \tau_{i}\right)^{2}}{1+\left(\omega \tau_{i}\right)^{2}} \\
G^{\prime \prime}(\omega)=\sum_{i=1}^{n} G_{i} \cdot \frac{\left(\omega \tau_{i}\right)}{1+\left(\omega \tau_{i}\right)^{2}}
\end{gathered}
$$

These constants can be replaced in the stress relaxation formula:

$$
\psi(t)=G_{e}+\sum_{i=1}^{n} G_{i} \exp \left(-t / \tau_{i}\right)
$$

The creep compliance function can then be calculated from the following relation [9], where s denotes the Laplace domain.

$$
\psi(s) \phi(s)=\frac{1}{s^{2}}
$$

The creep compliance curve in the time domain will also be described by Prony series with the form:

$$
\phi(t)=\phi_{r}-\sum \phi_{i} \exp \left(-\frac{t}{\lambda_{i}}\right)
$$

where $\phi_{r}$ corresponds to the creep compliance when the material is fully relaxed, $\phi_{i}$ creep compliance coefficients and $\lambda_{i}$ corresponds to the discrete retardation times. 



\section{Appendix G Full anisotropic contact model}

Willis [19] solves the effect of a point load on an anisotropic half space, and later uses the solution to solve the effect of a certain pressure distribution on an anisotropic half space.

The problem is defined by the stress equilibrium equation (where $\sigma$ indicates the stresses, repeated subscript denotes summation, comma denotes differentiation), the stress-strain equation for anisotropic materials and the boundary conditions.

$$
\begin{gathered}
\sigma_{i j, j}=0 \text { and } \sigma_{i j}=C_{i j k l} u_{k, l} \text { for } z \geq 0 \\
\sigma_{z z}=-\delta(x) \delta(y) F_{N} \quad \text { for } z=0
\end{gathered}
$$

Combining the two equations and introducing the convention that Latin suffixes take values of $x, y, z$ and Greek suffixes take values of $x, y$ :

$$
C_{i \alpha k \beta} u_{k, \alpha \beta}+\left(C_{i \alpha k z}+C_{i z k \alpha}\right) u_{k, \alpha z}+C_{i z k z} u_{k, z z}=0
$$

Taking the Fourier transform in the $x$ and $y$ directions, denoting $\xi=\left(\xi_{1}, \xi_{2}\right)$ as the Fourier transform parameters and $x=(x, y)$, the displacement in the Fourier domain is:

$$
\tilde{u}_{k}(\xi, z)=\sum_{A=1}^{6} a_{k^{A}}(\xi) \exp \left[i m_{A}(\xi) z\right]
$$

where

$$
\left[C_{i z k z} m_{A}^{2}+\left(C_{i \alpha k z}+C_{i z k \alpha}\right) \xi_{\alpha} m_{A}+C_{i \alpha k \beta} \xi_{\alpha} \xi_{\beta}\right] a_{k^{A}}=0
$$

$m_{A}$ is one of the six roots, obtained by setting the determinant of the matrix in Eq. G.5 to zero. As the displacement must be finite far from the load, only the roots with positive imaginary parts are retained. By substituting these roots back in Eq. G.5 the first two rows of the ratios $a_{1^{4}} / a_{3^{4}}$ and $a_{2^{A}} / a_{3^{4}}$ are obtained. By applying the Fourier transform to the boundary conditions and substituting Eq. G.4, the remaining constants are obtained:

$$
\sum_{A=1}^{3}\left[m_{A}\left(a_{1^{A}} / a_{3^{A}}\right)+\xi_{1}\right] a_{3^{A}}=0
$$




$$
\begin{gathered}
\sum_{A=1}^{3}\left[m_{A}\left(a_{2^{A}} / a_{3^{A}}\right)+\xi_{2}\right] a_{3^{A}}=0 \\
\sum_{A=1}^{3}\left[C_{x x z z} \xi_{1}\left(a_{1^{A}} / a_{3^{A}}\right)+C_{y y z z} \xi_{2}\left(a_{2^{A}} / a_{3^{A}}\right)-C_{z z z z} m_{A}\right] a_{3^{A}}=\frac{-i}{\pi}
\end{gathered}
$$

And the normal displacement in $z=0$ due to a concentrated normal load is known in its Fourier transform as:

$$
\tilde{u}_{z}(\xi, 0)=\sum_{A=1}^{3} a_{k^{A}}(\xi)
$$

By inverting this Fourier transform, assuming that the contact area is an ellipse with principal axes $\left(a_{x}\right.$ and $\left.a_{y}\right)$ and with a pressure distribution given by:

$$
p(x, y)=p_{0}\left(1-\frac{x^{2}}{a_{x}^{2}}-\frac{y^{2}}{a_{y}^{2}}\right)^{1 / 2}
$$

The normal displacement can be solved by numerically, by evaluating the following contour integral:

$$
\begin{aligned}
& u_{z}(x, y, 0)= \\
& p_{0} \frac{\pi a_{y}}{4} \int_{0}^{2 \pi} \widetilde{u}_{z}\left(\frac{a_{y}}{a_{x}} \cos \theta, \sin \theta\right) \cdot\left[1-\left(\frac{\cos \theta x}{a_{x}}+\frac{\sin \theta y}{a_{y}}\right)^{2}\right] d \theta
\end{aligned}
$$

The contact area can be expressed by:

$$
a_{x}=\left(\frac{3 R F_{N}}{4}\right)^{1 / 3} I_{1}^{1 / 3} \text { and } a_{y}=\left(\frac{3 R F_{N}}{4}\right)^{1 / 3} \frac{I_{2}^{1 / 2}}{I_{1}^{1 / 6}}
$$

where

$$
\begin{aligned}
& I_{1}=\int_{0}^{2 \pi} \widetilde{u}_{z}(\varepsilon \cos \theta, \sin \theta) \cdot(\cos \theta)^{2} d \theta \\
& I_{2}=\int_{0}^{2 \pi} \widetilde{u}_{z}(\varepsilon \cos \theta, \sin \theta) \cdot(\sin \theta)^{2} d \theta
\end{aligned}
$$

More details of this procedure can be found in Willis [19] and Swanson [20]. 


\section{References}

[1] Kaw, A.K., (1997), "Mechanics of Composite Materials", CRC Press, New York, NY.

[2] Mens, J.W.M., de Gee, A.W.J., (1991), "Friction and wear behaviour of 18 polymers in contact with steel in environment of air and water", Wear, 149, pp. 255-268.

[3] Blanchet, I.A., Kennedy, I.E., (1992), "Sliding wear mechanism of polyetrayuoroethylene (PTFE) and PTFE composites" Wear, 152, pp. 229-243.

[4] Shim, H.H., Kwon, O.K., Youn, J.R., (1992), "Effects of fibre orientation and humidity on friction and wear properties of graphite fibre composites", Wear, 157, 1, pp. 141-149.

[5] Lancaster, J.K., (1972), "Polymer based bearing materials, the role of fillers and fibre reinforcement", Tribology 5, 6, 249-255.

[6] Sung, N.H., Suh, N.P., (1979), "Effect of fiber orientation on friction and wear of fiber reinforced polymeric composites", Wear, 53, 1, pp. $129-141$.

[7] Wada, N., Uchiyama, Y., (1993), "Friction and wear of short-fibre reinforced rubber composites under various sliding speeds and loads", Wear, 162, 164, pp. 930-938.

[8] Uchiyama, Y.A., Wada, N.B., Iwai, T.A., Ueda, S.A., Sado, S.A., (2005), "Friction of short-fiber-reinforced rubber on wet surfaces", J. Appl. Polym. Sci., 95, 1, 82-89.

[9] Shaw, M.T., MacKnight, W.J., (2005), "Introduction to polymer viscoelasticity", John Wiley \& Sons, Inc., Hoboken, New Jersey.

[10] Johnson, K.L., (1985), "Contact Mechanics", Cambridge University Press, Cambridge.

[11] Lee, E.H., Radok, J.R.M., (1960), "The contact problem for viscoelastic bodies", J. Appl. Mech. 27, pp. 438-444.

[12] Graham, G.A.C., (1965), "The contact problem in the linear theory of viscoelasticity", Int. J. Eng Sci. 3, 27-46.

[13] Graham, G.A.C., (1967), "The contact problem in the linear theory of viscoelasticity when the time dependent contact area has any number of maxima and minima", Int. J. Eng Sci. 5, pp. 495-514. 
[14] Ting, T.C.T., (1966), "The contact stress between a rigid indenter and a viscoelastic half-space", J. Appl. Mech. 33, pp. 845-54.

[15] Green, A.E., Zerna, W., (1954), "Theoretical Elasticity", Oxford University Press, Oxford.

[16] Dahan, M., Zarka, J., (1977), "Elastic contact between a sphere and a semi infinite transversely isotropic body". Int. J. Solids. Struct. 13, pp. 229-238.

[17] Yang, S.H., Sun, C.T., (1982), "Indentation law for composite laminates". In: Daniel, I.M. (Ed.), Composite Materials: Testing and Design, 6th Conference. In: ASTM STP 787. pp. 425-449.

[18] Tan, T.M., Sun, C.T., (1985), "Use of statical indentation laws in the impact analysis of laminated composite plates". J. Appl. Mech. 5, pp. 6-12.

[19] Willis, J.R., (1966), "Hertzian contact of anisotropic bodies". J. Mech. Phys. Solids 14, pp. 163-176.

[20] Swanson, S.R., (2004), "Hertzian contact of orthotropic materials", Int. J. Solids Struct., 41, pp. 1945-1959.

[21] Bowden, F.P., Tabor, D., (1964), "The Friction and Lubrication of Solids", Clarendon Press, Oxford.

[22] Barquins, M., (1992), "Adherence, friction and wear of rubber-like materials", Wear 158, pp. 87-117.

[23] Roberts, A.D., (1976), "Theories of dry rubber friction", Tribol. Int. , 9, 2, pp. 75-81.

[24] Fukahori, Y., Gabriel, P., Busfield, J.J.C., (2010), "How does rubber truly slide between Schallamach waves and stick-slip motion?", Wear, 269, pp. 854-866.

[25] Arvanitaki, A., Briscoe, B.J., Adams, M.J., Johnson, S.A., (1995), "The Friction and Lubrication of elastomers" , In: D. Dowson, C.M. Taylor, T.H.C. Childs and G. Dalmaz, Editor(s), Tribology Series, Elsevier, 30, pp. 503-511.

[26] Vorvolakos, K., Chaudhury, M.J., (2003), "The effects of molecular weight and temperature on the kinetic friction of silicone rubbers", Langmuir 19, pp. 6778-6787. 
References

[27] Johnson, K.L., Kendall, K., Roberts, A.D., (1971), "Surface energy and the contact of elastic solids", P. Roy. Soc. Lond. A. 324, 1558, pp. 301-313.

[28] Derjaguin, B.V., Muller, V.M., Toporov, Y.P., (1975), "Effects of contact deformation on the adhesion of particles", J. Colloid Interface Sci. 53, 2, pp. 314-326.

[29] Maugis, D., (1992), "Adhesion of spheres: the JKR-DMT transition using a Dugdale model”, J. Colloid Interface Sci. 150, 1, pp. 243-269.

[30] Johnson, K.L., Greenwood, J.A., (1977), "An adhesion map for the contact of elastic spheres", J. Colloid Interface Sci. 192, 2, pp. 326333.

[31] Bhushan, B., (1999), "Principles and applications of tribology", Wiley, New York.

[32] Barquins, M., Roberts, A. D., (1986), "Rubber friction variation with rate and temperature: some new observations" J. Phys. D: Appl. Phys. 19, pp. 547-563.

[33] Grosch, K. A., (1963), "The Relation between the Friction and ViscoElastic Properties of Rubber", Proc. R. Soc. Lond. A. 274, 1356, pp. 21-39.

[34] Clark, S.K., (1981), "Mechanics of Pneumatic Tires", U. S. Department of Transportation, National Highway Traffic Safety Administration, Washington DC.

[35] Roth, F.L., Driscoll, R.L., Holt, W.L., (1942), "Frictional properties of rubber" J. Res. Nat. Bur. Stds. 28, 4, pp. 439-462.

[36] Smith, R. H., (2011), "A Load-Independent, Adhesion-Related Rubber Friction Force?", J. Adhesion 87, 11, pp. 1112-1123.

[37] Sinha, S. K., Briscoe, B.J., (2009), "Polymer tribology", Imperial College Press, London.

[38] Chan, C.M., (1994), "Polymer surface modification and characterization", Hanser, New York.

[39] Andrade, J.D., (1988), "Polymer surface dynamics", Plenum, New York.

[40] Garbassi, F., Morra M., Occhiello, E., (1994), "Polymer surfaces: from physics to technology", Wiley, Chichester. 
[41] Lei, Y.G., Cheung, Z.L., Ng, K.M., Li, L., Weng, L.T., Chang, C.M., (2003), "Surface chemical and morphological properties of a blend containing semi-crystalline and amorphous polymers studied with ToF-SIMS, XPS and AFM", Polymer 44, 14, 3883-3890.

[42] Toney, M.F., Russell, T. P., Logan, J.A., Kikuchi, H., Sands, J.M., Kumar S.K., (1995), "Near-surface alignment of polymers in rubbed films". Nature 374, 709-711.

[43] Karger-Kocsis, J., Mousa, A., Major, Z., Békési, N., (2008), "Dry friction and sliding wear of EPDM rubbers against steel as a function of carbon black content", Wear 264, 3-4, 359-367.

[44] Martínez, L., Nevshupa, R., Felhös, D., de Segovia, J.L., Román, E., (2011), "Influence of friction on the surface characteristics of EPDM elastomers with different carbon black contents", Tribol. Int. 44, 9, pp. 996-1003.

[45] Thomine, M., Degrange, J-M, Vigier, G. , Chazeau, L., Pelletier, J.-M., Kapsa, P., Guerbe, L., Dudragne,G., (2007), "Study of relations between viscoelasticity and tribological behaviour of filled elastomer for lip seal application", Tribol. Int. 40, 2, pp. 405-411.

[46] Deladi, E.L., (2006), "Static friction in rubber-metal contacts with application to rubber pad forming processes". PhD thesis, University of Twente, Enschede, The Netherlands.

[47] Ferry, J.D, (1980), "Viscoelastic Properties of Polymers", John Wiley and Sons.

[48] Schapery, R.A., Park, S.W., (1999), "Methods of interconversion between linear viscoelastic material functions. Part II-an approximate analytical method", Int. J. Solids Struct. 36, 11, pp.16771699.

[49] Matsuzaki, R., Todoroki, A., (2005), "Wireless strain monitoring of tires using electrical capacitance changes with an oscillating circuit", Sensor Actuat. A.-Phys. 119, 2, pp. 323-331.

[50] Heinrich, G., Klüppel, M., (2008), "Rubber friction, tread deformation and tire traction", Wear 265, pp. 1052-1060.

[51] Maugis, D., (2000), "Contact, Adhesion and Rupture of Elastic Solids", Springer, Berlin. 
[52] Perriot, A., Barthel, E., (2004), "Elastic contact to a coated halfspace: Effective elastic modulus and real penetration", J. Mater. Res. 19, 2, pp. 600-608.

[53] Chen W.T., (1969), "Stresses in some anisotropic materials due to indentation and sliding", Int. J. Solids Struct. 5, pp. 191-214.

[54] Lu, H., Wang, B., Ma, J., Huang, G., Viswanathan, H., (2003), "Measurements of creep compliance of solid polymers by nanoindentation", Mech. Time-Depend. Mat. 7, pp. 189-207.

[55] Hutapea, P., Grenestedt, J., (2003), "Effect of temperature on elastic properties of woven-glass epoxy composites for printed circuit board applications", J. Electron. Mater. 32, 4, pp. 221-227.

[56] Shrotriya, P., Sottos, N.R., (1998), "Creep and relaxation behavior of woven glass/epoxy substrates for multilayer circuit board applications", Polym. Compos. 19, 5, pp. 567-578.

[57] Schallamach, A., (1952), "The velocity and temperature dependence of rubber friction", Proc. Phys. Soc. B 65, pp. 657-661.

[58] Schallamach, A., (1952), "The load dependence of rubber friction", Proc. Phys. Soc. B 66, pp. 386-392.

[59] Ghatak, A.., Vorvolakos, C., She, H., Malotky, D.L., Chaudhury, M.K., (2000), "Interfacial Rate Processes in Adhesion and Friction", J. Phys. Chem. B. 104, pp. 4018-4030.

[60] She, H., Malotky, D.L., Chaudhury, M.K., (1998), "Estimation of Adhesion Hysteresis at Polymer/Oxide Interfaces Using Rolling Contact Mechanics", Langmuir 14, pp. 3090-3010.

[61] Lorenz, B., Persson, B.N.J., Dieluweit, S., Tada, T., (2011), "Rubber friction: Comparison of theory with experiment", Eur. Phys. J. E 34: 129.

[62] Greenwood, J.A., Johnson, K.L., Matsubara, E., (1984), "A surface roughness parameter in Hertz contact", Wear 100, pp. 47-57.

[63] Fischer-Cripps, A.C., (2004), "Nanoindentation”, Springer, New York.

[64] Tweedie, C.A., Van Vliet, K.J., (2006), "Contact creep compliance of viscoelastic materials via nanoindentation", J. Mater. Res. 21, 6, pp. 1576-1589. 
[65] Oliver, W.C., Pharr, G.M., (1992), "An Improved Technique for Determining Hardness and Elastic Moduli using Load and Displacement Sensing Indentation Experiments.", J. Mater. Res. 7, 6, pp. 1564-1583.

[66] Popovici, R., (2010), "Friction in Wheel-Rail Contacts", PhD thesis, University of Twente, Enschede, The Netherlands.

[67] Bair, S. and Winer, W.O., (1979), "A rheological model for elastohydrodynamic contacts based on primary laboratory data", J. Lubric. Tech.-T. ASME 101, pp. 258-265.

[68] Gecim, B. and Winer, W.O., (1980), "Lubricant limiting shear stress effect on EHD film thickness", J. Lubric. Tech.-T. ASME 102, pp. 213221.

[69] Elsharkawy, A.A. and Hamrock, B.J., (1991), "Subsurface stresses in micro-EHL line contacts”, J. Tribol.-T. ASME 113, pp. 645-657.

[70] Johnson, K.L. and Roberts, A.D. (1974), "Observations of viscoelastic behaviour of an elastohydrodynamic lubricant film", Proc. Roy. Soc. Lond. A. 337, 1609, pp. 217-249.

[71] Ludema, K.C., Tabor, D., (1966), "The friction and visco-elastic properties of polymeric solids", Wear 9, pp. 329-348.

[72] Wu S., (1971), "Calculation of interfacial tension in polymer systems." J. Polym. Sci. Pol. Lett. 34, 1, pp. 19-30. 


\section{Publications}

Rodriguez, N., Masen, M.A., Schipper, D.J., (2012), "Tribologically modified surfaces on elastomeric materials", accepted in P. I. Mech. Eng. J-J Eng.

N.V. Rodriguez, M.A. Masen, D.J. Schipper, (2012), "A Model for the Contact Behaviour of Weakly Orthotropic Viscoelastic Materials.", submitted to Int. J. Mech. Sci. (2012)

N.V. Rodriguez, M.A. Masen, D.J. Schipper, (2012), "A Contact Model For Anisotropic Viscoelastic Materials", submitted to Int. J. Mech. Sci.

N.V. Rodriguez, M.A. Masen, D.J. Schipper, (2012), "Adhesive friction in tribo-systems with elastomeric materials", submitted to Wear

N.V. Rodriguez, M.A. Masen, D.J. Schipper, (2012), "The variation of the contact area of viscoelastic materials as a function of sliding velocity", submitted as Short Communications in Wear. 